\title{
YOUTH DEVELOPMENT APPROACHES IN ADOLESCENT FAMILY LIFE DEMONSTRATION PROJECTS
}

\section{FINAL REPORT}

\author{
Prepared by \\ Martha R. Burt \\ Jeffrey Capizzano \\ Janine M. Zweig \\ Shinta Herwantoro \\ Alexandra Fiorillo
}

\author{
Submitted to \\ Office of Adolescent Pregnancy Programs \\ U.S. Department of Health and Human Services \\ The Tower Building at Tower Oaks \\ 1101 Wootton Parkway, Suite 700 \\ Rockville, MD 20852
}

Contract No. 233-02-0092

Task Order No. 8

Urban Institute No. 07417-008-00

Task 2.2

\author{
Submitted by \\ The Urban Institute \\ 2100 M Street, NW \\ Washington, DC 20037
}

September 21, 2005

The contents of this report are the views of the authors and do not necessarily reflect the views or policies of the Office of Population Affairs, Department of Health and Human Services, or of the Urban Institute, its trustees, or funders. 


\section{TABLE OF CONTENTS}

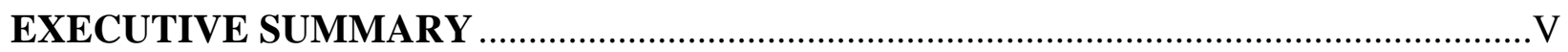

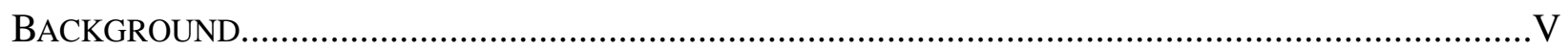

RESEARCH QUESTIONS AND METHODS ............................................................................. VI

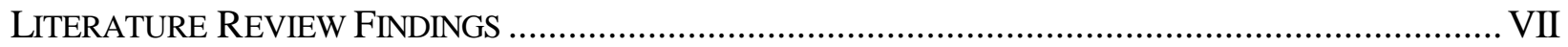

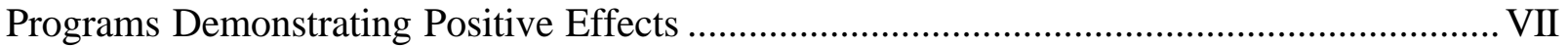

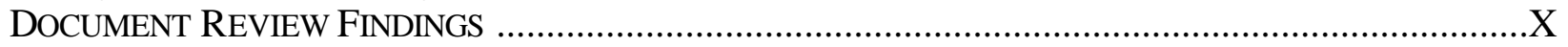

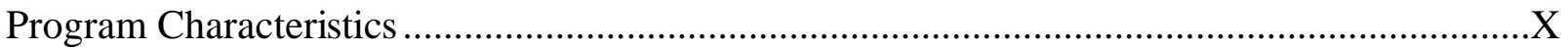

Positive Youth Development Objectives ................................................................................

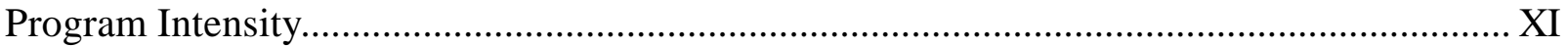

Challenges Facing AFL Grantees ...................................................................................... XII

PROGRAMMATIC FINDINGS FROM SITE VISITS ...................................................................... XII

FINDINGS FROM REVIEWS OF AFL GRANTEE EVALUATIONS .....................................................III

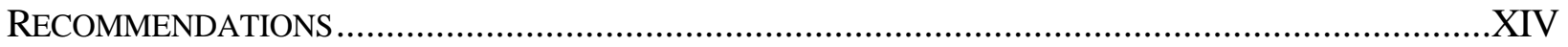

Strengthening the Usefulness of year-End Reports …......................................................

Strengthening Individual Program Evaluations ..............................................................

Strengthening OAPP's Ability to Assess Effectiveness Across Grantees ..............................XVI

CHAPTER 1: INTRODUCTION AND OVERVIEW OF THE REPORT........................... 1

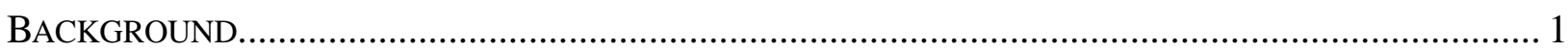

RESEARCH QUESTIONS AND METHODS ............................................................................ 2

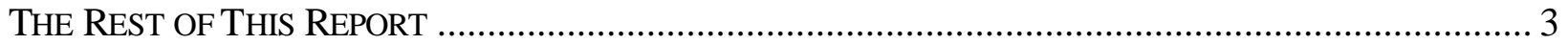

\section{CHAPTER 2: REVIEW OF RESEARCH ON EFFECTIVENESS OF INTERVENTIONS TO PROMOTE ABSTINENCE, WITH SPECIAL ATTENTION TO THE ROLE OF

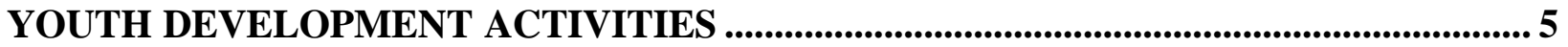

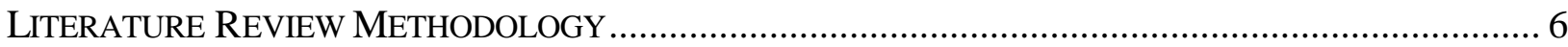

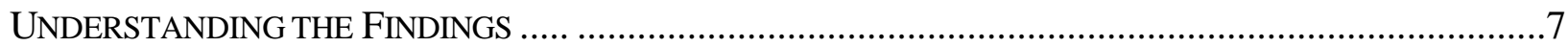

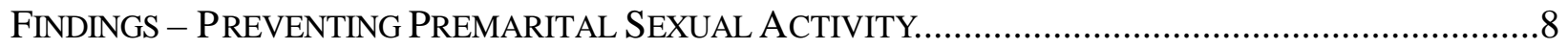

The Programs Demonstrating Positive Effects.............................................................. 10

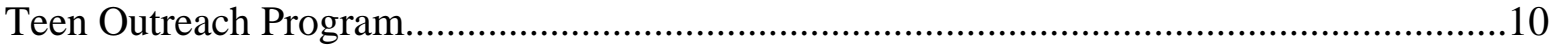

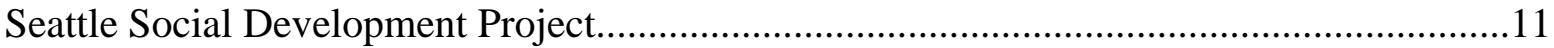

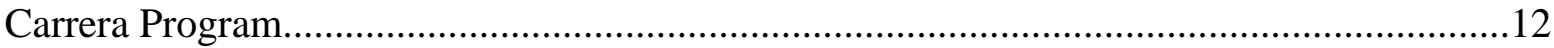

Client-Centered Intervention Approach.....................................................................13

Reach for Health Community Youth Service Learning Program...................................... 14

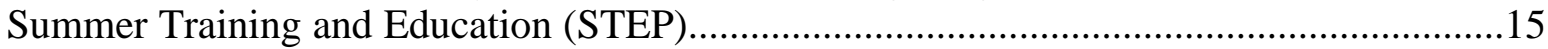

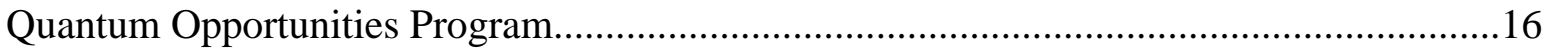

FINDINGS - STRENGTHENING PROTECTIVE ASSETS........................................................... 16

PROTECTIVE FACTORS THAT DELAY FIRST SEX............................................................. 17 


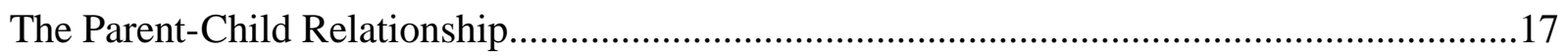

Positive Emotional Development................................................................................19

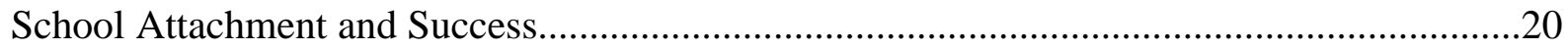

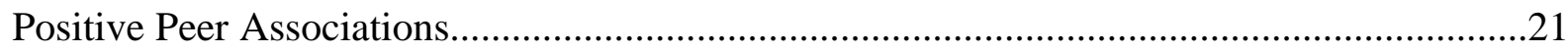

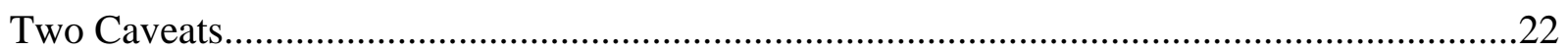

THE IMPACT OF YOUTH DEVELOPMENT PROGRAMS ON PROTECTIVE FACTORS THAT DELAY

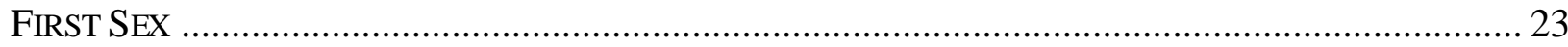

Two Comprehensive Reviews of Multidimensional YD Programs...........................................22

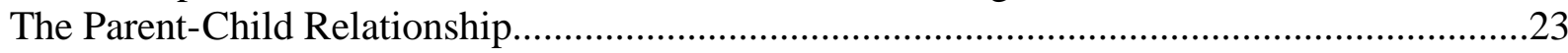

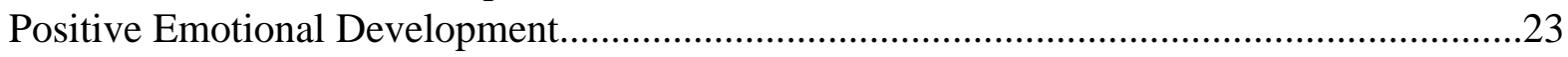

School Attachment and Success.................................................................................23

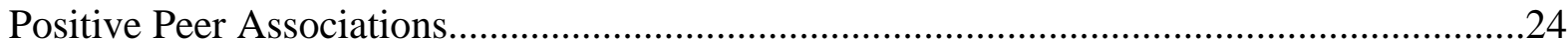

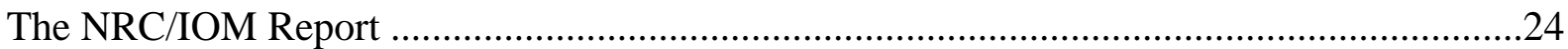

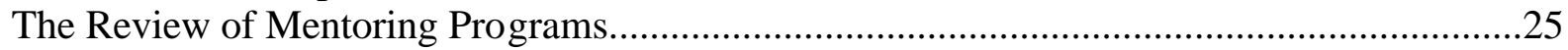

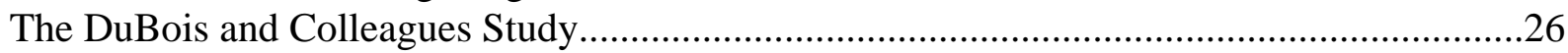

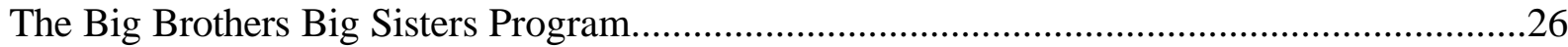

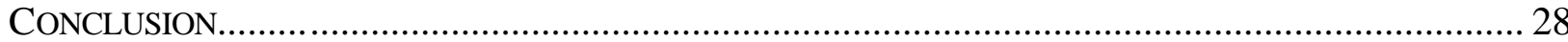

\section{CHAPTER 3: REVIEW OF YOUTH DEVELOPMENT COMPONENTS OF OAPP-} FUNDED ABSTINENCE PROJECTS ................................................................................... 29

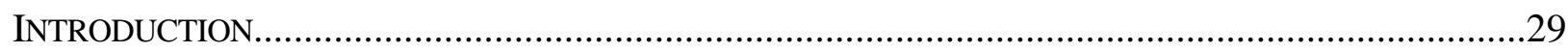

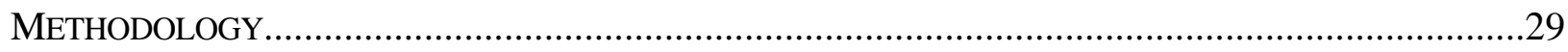

DEFINING PoSITIVE YOUTH DEVELOPMENT OBJECTIVES ..............................................................

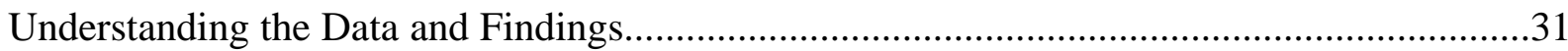

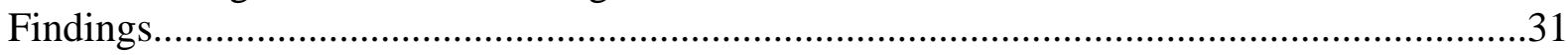

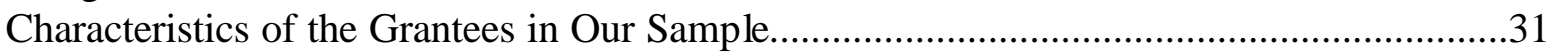

Assessment of Positive Youth Development Objectives...........................................................33

Observed Positive Youth Development Objectives...................................................................34

Approaches to Positive Youth Development Objectives.............................................................34

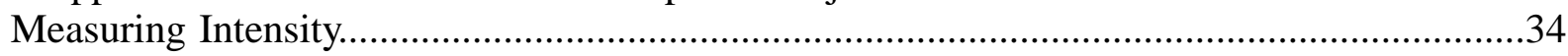

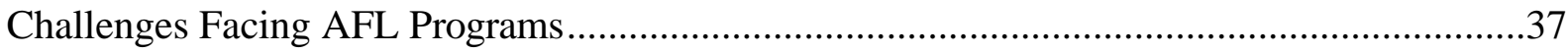

IMPLICATIONS OF THE DOCUMENT REVIEW FOR AFL PROGRAM EVALUABILITY..............................40

Understanding How the AFL Grantees Are Evaluated.......................................................... 41

Dimensions of a Strong Evaluation Design............................................................................40

Do the evaluations demonstrate a strong understanding of the intervention components and how they may affect outcomes?................................................................41

How do the evaluations vary in their design?................................................................42

Do the evaluations measure the extent to which the participants are exposed to the intervention components? ......................................................................................42

How well did the evaluations measure and collect data on specific child outcomes, particularly those related positive youth development objectives?.......................................43

Overall assessment of program eva luations...........................................................................4

CHAPTER 4: SITE VISITS....................................................................................................45 
PeEr LEAder Program, People’s Regional OpPortunity Program-Portland, ME........45

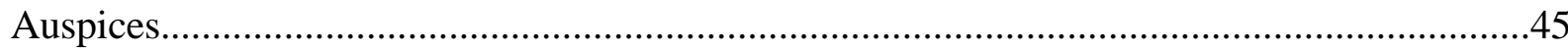

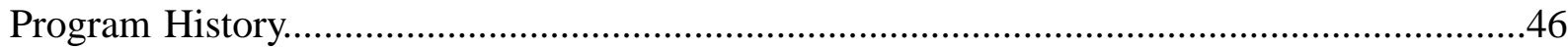

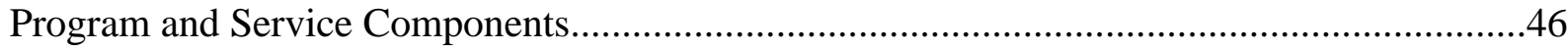

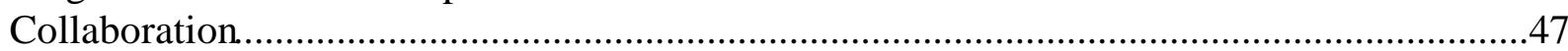

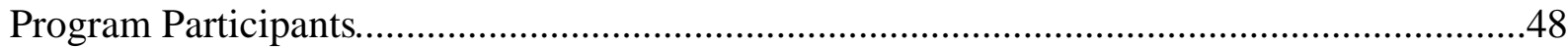

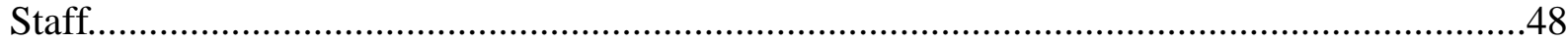

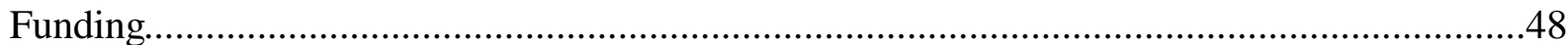

ThE REAL DEAL (TRD), BE'ER HagOlah InSTITUTES-BROOKLYN, Ny .............................. 49

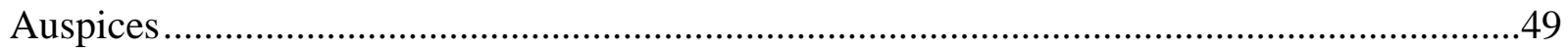

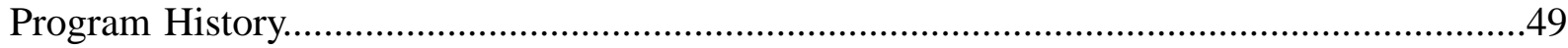

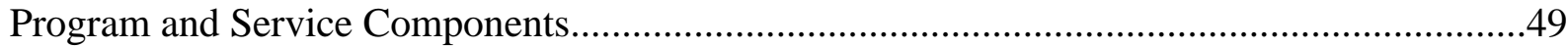

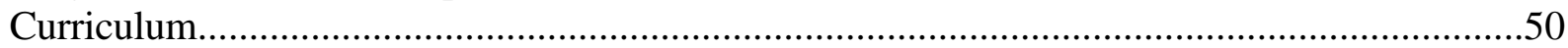

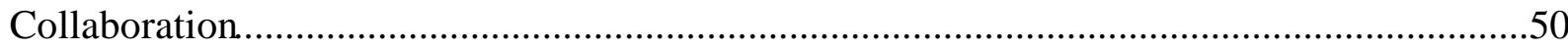

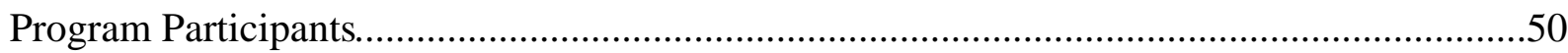

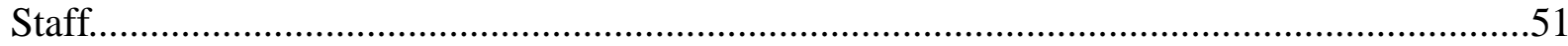

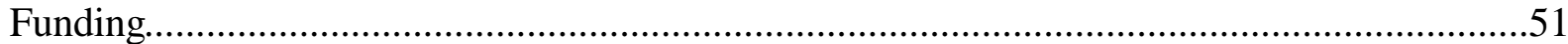

LEADERSHIP 101, CROZER-CHESTER MEDICAL CENTER_CHESTER, PA...................................51

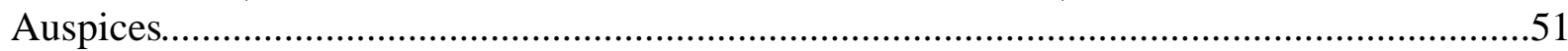

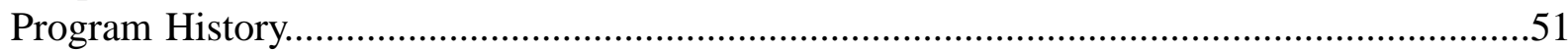

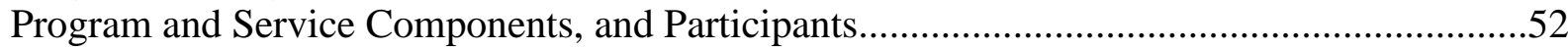

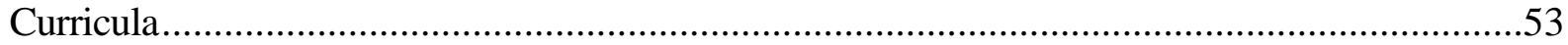

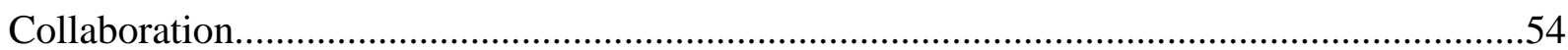

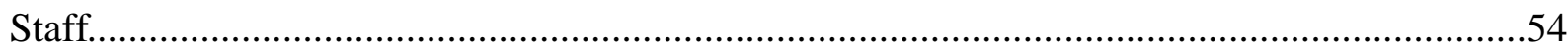

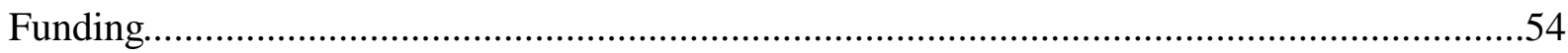

TEACHING RESPONSIBLE ACTIONS IN LIFE (TRAIL), PUBLIC HEALTH AUTHORITY OF CABARRUS

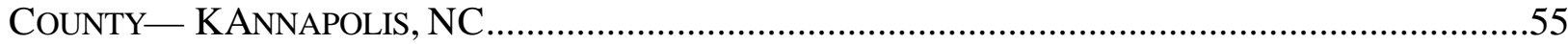

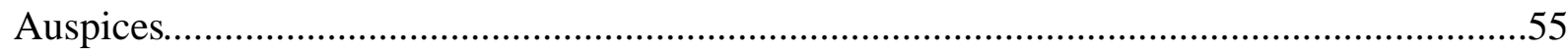

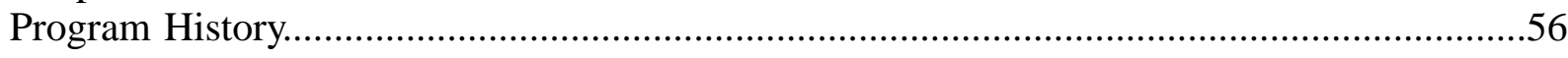

Program and Service Components, and Participants........................................................56

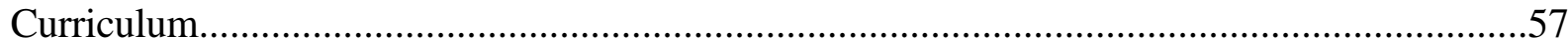

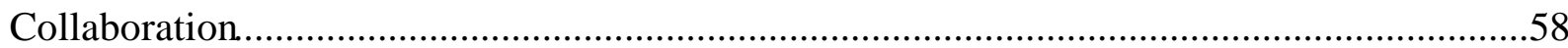

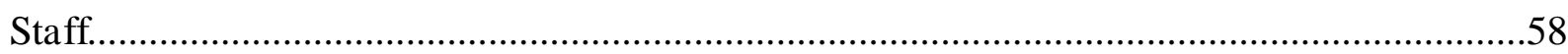

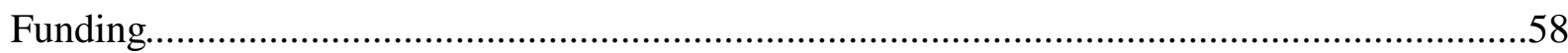

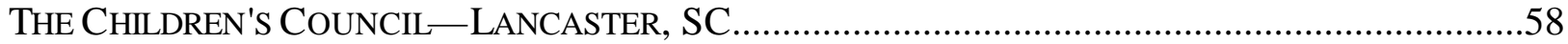

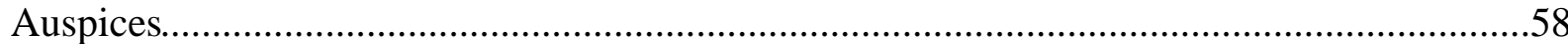

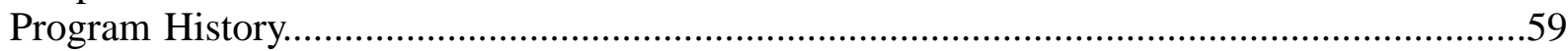

Program and Service Components, and Participants...........................................................59

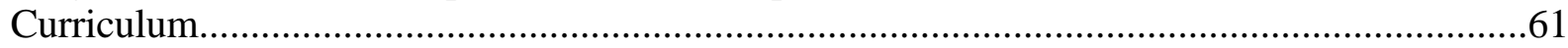

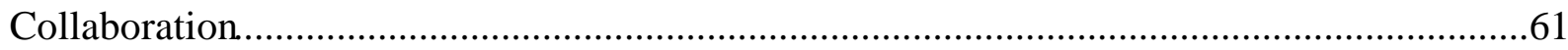

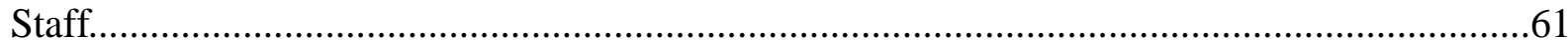

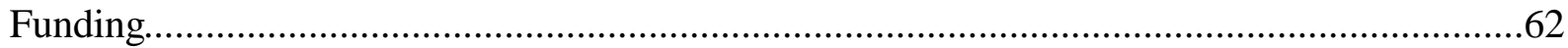

FAME (FAMILY ACTION MODEL FOR EMPOWERMENT), BETA CENTER —ORLANDO, FLORIDA..62

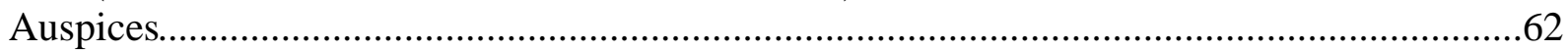

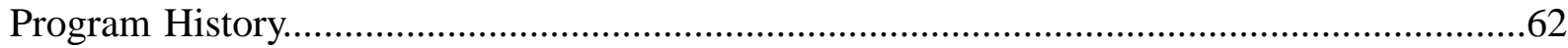

Program and Service Components, and Participants.......................................................63 


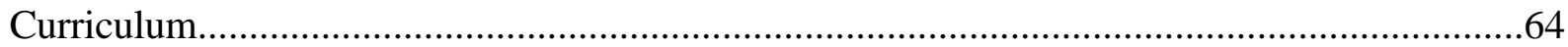

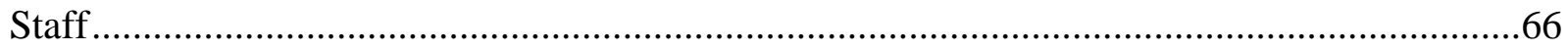

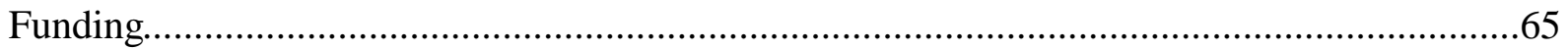

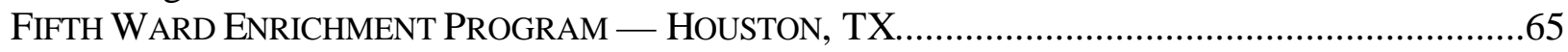

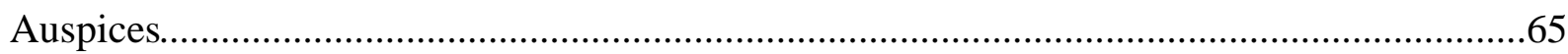

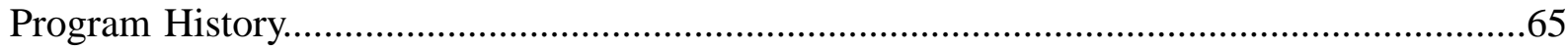

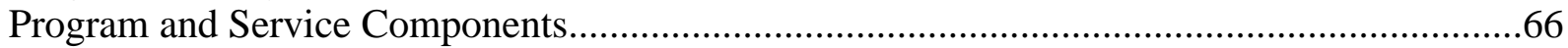

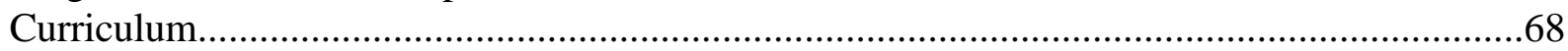

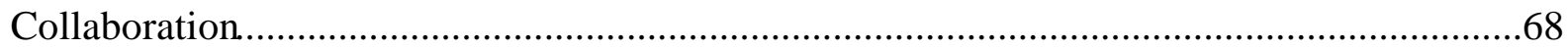

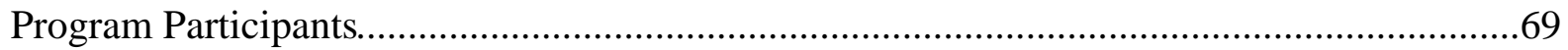

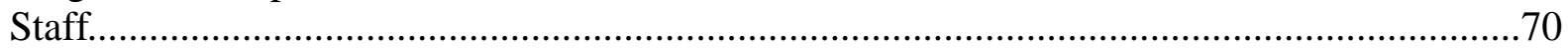

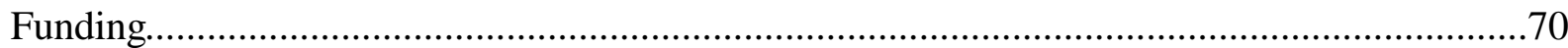

INTEGRATION OF ABSTINENCE AND YD COMPONENT.............................................................

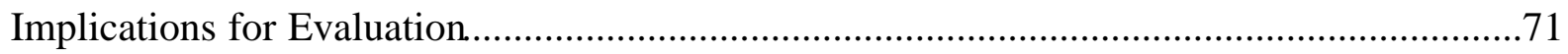

CHAPTER 5: EVALUATING ABSTINENCE AND YD COMPONENTS OF AFL-

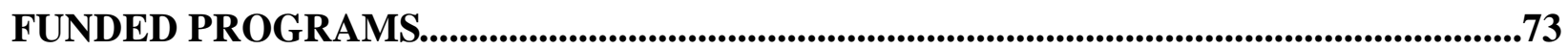

STRENGTHENING THE USEFULNESS OF YEAR-END REPORTS.......................................................73

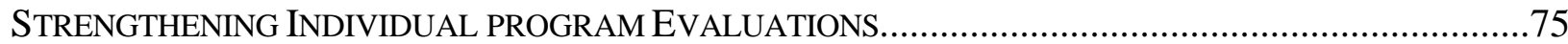

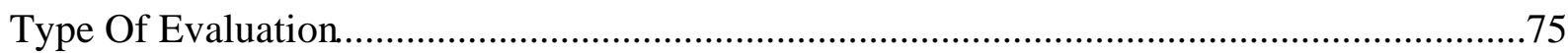

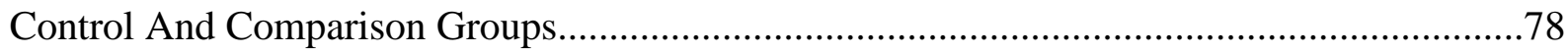

Types Of Questions And Topics Tested...............................................................................79

Other Long-Term Indicators Currently Being Tested................................................................8

Analyses Reported And Other Opportunities To Understand Program Effects.........................80

Program-Secific Recommendations For Improving Evaluations............................................... 81

Implications For Improving Other Individual AFL Grantee Evaluations..................................83

STRENGTHENING OAPP's ABILITY TO ASSESS EFFECTIVENESS ACROSS GRANTEES.......................84

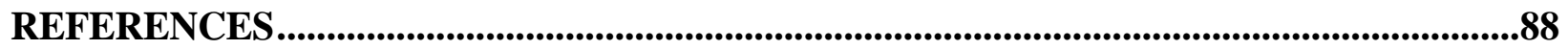

APPENDIX A, PART 1: YOUTH DEVELOPMENT IMPACTS ON TEENS' SEXUAL

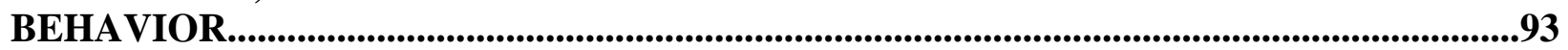

APPENDIX A, PART 2: YOUTH DEVELOPMENT IMPACTS ON THE

ANTECEDENTS OF FIRST SEX...................................................................................................94

APPENDIX B: DOCUMENT REVIEW PROTOCOL...............................................................95

APPENDIX C: CODING YOUTH DEVELOPMENT OBJECTIVES ................................. 103

APPENDIX D, PART 1: SUMMARY OF DOCUMENT REVIEWS .................................... 106

APPENDIX E: SUGGESTED ANNUAL REPORT OUTLINE............................................... 114 


\section{EXECUTIVE SUMMARY}

\section{BACKGROUND}

"How can adolescents be better connected to their families and schools, and will these connections result in decreased sexual activity?" "What effective practices can [we] use to assist adolescents and young adults in sexual decisionmaking?" A panel of experts articulated these questions in "Future Directions in Family Planning Research" (Sonenstein, Punja, and Scarcella 2004, p.37), a study sponsored by the Office of Population Affairs. The panel assigned high priority to identifying successful approaches to address the sexual and reproductive needs of adolescents. Despite continuing drops in births to teenagers, the teen birthrate in the United States is still one of the highest among developed countries. The social and financial costs of early childbearing still generate considerable public concern.

Youth development (YD) strategies in conjunction with appropriate age-graded sexuality and family life education programs/curricula may have an important role to play in formulating convincing answers to these questions. Youth development approaches help youth enhance their assets rather than concentrating on their difficulties. They focus on where youth are going, helping them develop a belief in a viable future and in their ability to take actions that will bring that future about. The commitment to a future that would be disrupted by a pregnancy during adolescence is about the only thing that Zabin and her colleagues (1986) found to differentiate among Baltimore adolescents using teen clinics who did and did not get pregnant. Teens without a strong reason to avoid pregnancy got pregnant at the same rate as those who wanted to get pregnant; the only teens who were successful at avoiding pregnancy were those who had a future goal that a pregnancy would disrupt. Thus, incorporating youth development principles along with some specific techniques into the work of the Office of Adolescent Pregnancy Programs' (OAPP) abstinence-oriented programs would seem to be an important program enhancement with potentially valuable impacts.

Adding further impetus to OAPP's interest in conducting useful evaluations is an everstrengthening emphasis throughout government and among prevention scientists on performance measurement and use of strong scientific evidence to aid decisions about funding, implementation, and extension of social, health, and educational intervention programs and services. A small number of evaluations of comprehensive programs (denotated "Abstinence Plus" curricula) based on strong experimental or quasi-experimental designs appear to be relatively effective in postponing first intercourse, decreasing the number of partners, increasing contraceptive use, and/or decreasing the incidence of pregnancy, births, and STDs (reviews by Kirby 2001; Manlove, Franzetta, et al. 2004; Manlove, Romano-Papillo, \& Ikramullah 2004). Currently, the evidence base for abstinence-only programs - the type of program under review in this report-is more equivocal. Because these programs are newer, rigorous evaluations have only recently been fielded. Further, many of the most promising programs and approaches target preadolescents, in an attempt to reach teens before they initiate sexual activity and while their attitudes and intentions may be more amenable to influence. This age targeting is appropriate, given what research tells us about many interventions (captured in the phrase "too little, too late"). But from the perspective of trying to evaluate the impact of these programs, the short span 
of most follow-up data collection schedules (i.e., 6-24 months postintervention) will not capture the times when these participants begin to experience dating and sexual risk-taking situations.

\section{RESEARCH QUESTIONS AND METHODS}

In the face of compelling evidence that teen birth rates declined during the same period that implementation of abstinence-only programs/curricula and youth development/developmental assets approaches proliferated, and also stimulated by the accountability pressures just described, OAPP asked Urban Institute researchers to examine the documented relationships among provision of abstinence education together with YD programming as they might affect developments and changes in teen sexual risk-taking behaviors as well as pregnancy prevention. The research reported here is intended as a strong first step in the investigative process. Specifically, it sought to answer to the following questions:

1. What can be learned from a comprehensive review of the relationships identified in research between YD activities and prevention of sexual risk taking?

2. What additional information can be learned from a careful and systematic review of the year-end reports of current Adolescent Family Life (AFL) grantees who are using YD activities in their intervention programs, augmented by input from OAPP project officers?

3. What additional information, insights, and empirical relationships might be learned about adherence to program models and materials (i.e., program fidelity) and sexualityrelated outcomes for the combined YD and abstinence education approaches delivered in the most "evaluable" AFL sites?

The project had as its ultimate aim helping OAPP by answering these questions in a way that provides strong guidance to the next generations of AFL grantees and their evaluators. Issues include the importance/salience of including specific YD program concepts, elements, and components in OAPP intervention programs and services to increase the synergy with and potential effectiveness of abstinence education programs; and ways to evaluate these enhanced programs to show the effects of augmenting abstinence education with YD approaches.

Three different methods were used to answer the research questions.

- Literature review. Project staff reviewed all recent literature pertaining to abstinence or delay of sexual activity, with special attention to how factors that are the target of youth development approaches have been shown to affect these outcomes.

- Document review. Project staff reviewed the year-end reports and evaluation reports for all AFL-funded projects that had submitted at least one such report by December 2004.

- Site visits. On the basis of document reviews and AFL project officers' advice, project staff selected seven AFL grantees that appeared to be using YD approaches extensively, 
and visited them to learn in detail how they structure their programs to include both abstinence education and strategies to promote youth development.

\section{LITERATURE REVIEW FINDINGS}

This project reviewed literature pertaining to the effectiveness of YD strategies in helping to prevent adolescent premarital sexual activity and pregnancy. The review had two purposes. First, it summarized the results of evaluations that show statistically significant relationships between specific YD approaches and the delay of first sexual intercourse and pregnancy prevention. Second, because many YD program evaluations do not measure the specific outcomes of most interest to OAPP, the review also summarized research findings concerning the effects of selected YD strategies on some salient antecedents associated with higher or lower levels of early sexual intercourse and adolescent pregnancy. Given that these antecedents are well researched and documented, understanding how YD strategies may affect them is important to understanding how these approaches may help to delay the onset of sexual activity.

To guide the review, research staff constructed a generic logic model, showing the presumed connections among program interventions and the outcomes they are expected to achieve. As we reviewed each program evaluation, we compared its assumptions and expected pathways of influence to this generic logic model, which let us view each program within a single overall intervention framework. Figure ES.1 shows the entire generic model, highlighting the pathways for which we sought evidence in the literature review.

\section{Programs Demonstrating Positive EfFects on R Educed Sexual Risk Taking}

The literature review identified programs whose evaluations documented direct effects of YD approaches on sexual risk taking, and additional programs designed to improve youth status on several social domains (antecedents), which in turn were expected to reduce or prevent sexual risk-taking behavior. All of these studies have several common elements.

- They were clear about their intervention(s) and how they were expected to produce desired outcomes. That is, they had clear logic models.

- Evaluations of these programs measured outcomes as they related to the distinguishable YD activities set forth in their programs - that is, their evaluations were designed to assess the accuracy and adequacy of their logic models to identify what worked and why.

- One or more social domains were addressed through various interventions;

- Substantial numbers of participants were in both the treatment and comparison or control groups;

- They included YD activities (e.g., community service learning, parent-child communication workshops);

- They performed pre- and posttest of participants to assess short-term changes;

- They performed longitudinal or follow-up testing of participants to assess longer-term effects; and

- They showed positive results in desired outcomes (e.g., decrease in sexual risk-taking behavior, increase in knowledge of and attitude towards problem behaviors in addition to 
ES.1: Youth Development (YD) and Abstinence Education (AE) Program Evaluation Model

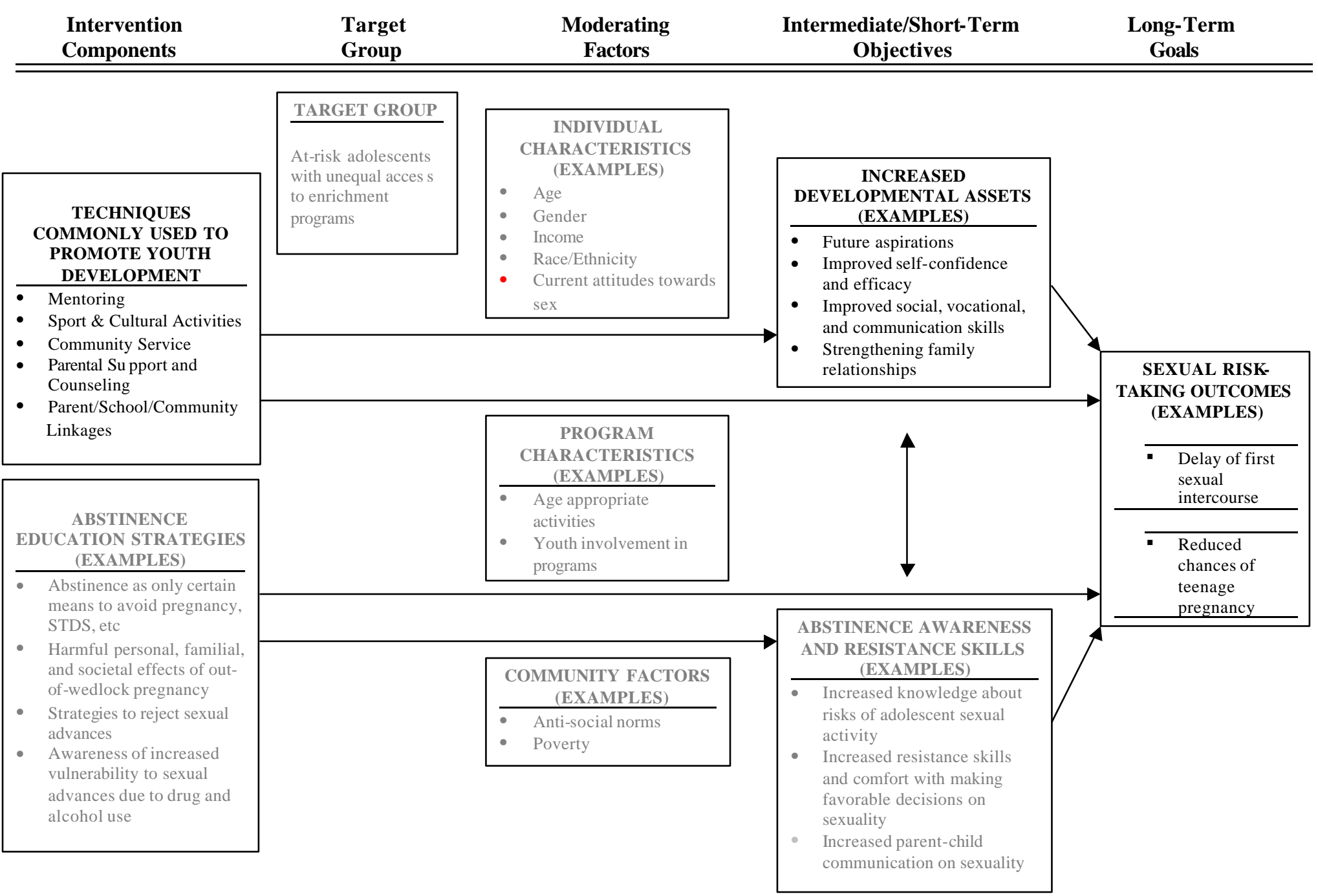


improvement in actual behavior, increased academic achievement, improved parent-child communication, etc.)

The first set of programs, which were able to document specific effects on sexual risk-taking behaviors, showed the following positive results:

- lower chance of teenage pregnancy;

- decreased level of sexual activity;

- lower number of sexual partners;

- increased knowledge about responsible decisionmaking, contraception, and other aspects of sexual behavior; and

- lower chance of school failure and academic suspension.

Another set of evaluations did not assess sexual behavior directly, but did assess program effects on antecedents of sexual behavior. These programs generally show positive results, suggesting that those who receive the intervention(s) are more likely to increase their probability of remaining abstinent. Four social domains were found to respond to program interventions that are expected to affect delay in first sex, delay in other sexual risk taking, or both:

- Strength of the parent-child relationship. The more teens feel close to their parents, the more likely they are to perceive parental disapproval and to feel that they want to avoid it. Programs were successful in strengthening parent-child relationships, thereby increasing the perceived costs of parental disapproval and leading teens to avoid sexual activity. To do this, they focused at least as much on the parents as on the youth; tried to improve communication around all topics, not just sexuality; and created opportunities for joint parent-teen involvement in community activities.

- Positive emotional development. To increase two elements of positive emotional development, self-esteem, and planfulness (future orientation with realistic steps to achieve goals), programs provided opportunities to succeed at meaningful goals, recognized youth achievements, worked on cohesion and trust building, helped youth with concrete barriers through tutoring and language development, and focused on nonmainstream youth with multiple barriers to success. Youth with concrete plans for the future are less likely to choose risky sexual or other behavior that might jeopardize the plans, and those with high self-esteem will have enough confidence not to need sex to tell themselves they are all right.

- School attachment and success. School attachment involves integration with school life, perceived fairness of school structures and activities, and closeness to teachers and other school personnel. School attachment tends to promote success in school, which in turn supports future educational aspirations and increases the perceived cost of sexual activity.

- Positive peer associations . Building teens' strong supports for each other to avoid risky sexual and other behaviors strengthens resistance to negative neighborhood and school influences. 


\section{DOCUMENT REVIEW FINDINGS}

Researchers reviewed year-end reports and evaluation reports for 45 out of approximately 60 AFL grantees. Programs reviewed included all grantees for which OAPP had relevant documentation by the end of December 2004. Reviews covered general program characteristics, positive youth development objectives included in program designs, methods for achieving objectives, and program challenges.

\section{Program Characteristics}

Projects reviewed used different program configurations. Relatively few (7 percent) were schoolbased, 44 percent were after-school only, 27 percent were after-school and summer, 18 percent were summer only, and 4 percent had other configurations or information was insufficient to classify them.

Most programs (40 percent) served youth from mixed racial and ethnic backgrounds. Twentyfour percent served predominantly African-American youth, 11 percent served mostly Latinos, 7 percent each served mostly white and mostly Native American youth, and the rest for which information was reported served a variety of different populations. Seven percent were missing this information.

The vast majority of programs ( 91 percent) served both boys and girls. Two percent served boys only, and 7 percent served girls only.

\section{Positive Youth DeVELOPMENT OBJECTIVES}

Researchers identified 16 positive youth development objectives from available literature (Catalano et al. 2002, 2004), including:

- promoting bonds with prosocial institutions and community;

- promoting bonds with parents/family and parent/child communication;

- fostering resilience;

- promoting social, emotional, cognitive, behavioral, and moral competence (5 objectives);

- fostering self-determination;

- fostering spirituality;

- promoting self-efficacy;

- promoting a clear and positive identity;

- fostering a belief in a future;

- recognizing and reinforcing positive behavior;

- providing opportunities for pro-social involvement; and

- enhancing pro-social norms.

AFL grantee documents were reviewed for evidence that programs incorporate one or more of these objectives. Three programs targeted 15 of the 16 objectives, and another twelve programs targeted 13 or 14 objectives. Other programs were less inclusive, with several programs 
apparently incorporating very few positive youth development objectives. ${ }^{1}$ The most commonly targeted youth development objectives were promoting school/community bonding, promoting parent/child communication and bonding, fostering resilience, fostering self-efficacy, and fostering cognitive competencies. The least commonly mentioned objective was fostering spirituality. AFL grantees used two primary approaches to deliver their abstinence and youth development messages - curriculum-based activities and enrichment activities.

Curriculum-based activities occurred in school classrooms and involved use of a specific curriculum, curriculum-based discussion, role plays, films, and movies. Some programs used modifications of these techniques in nonschool settings, taking greater liberties in molding curriculum messages to their unique populations and delivering them in ways they learned from experience were the most likely to be effective.

Enrichment activities spanned many forms, including peer and adult mentoring; annual and special events; athletics; performance art (putting on plays, music, doing art, writing prose and poetry); entrepreneurial training and experience; and day, overnight, and summer camp. Many programs also offered opportunities to serve the community through volunteer work, clean-up, construction, arts, and many other activities. The point of most enrichment activities was to provide opportunities for youth to set goals and achieve them, thereby building both skills and self-confidence. Programs often used some of the time spent in enrichment activities to discuss future plans, risky behaviors, and the likelihood that engaging in risky behaviors would jeopardize future plans.

\section{Programintensity}

Research staff were interested in examining the intensity of AFL grantee programs because much of the literature speaks to the importance of early starts and multiyear continuity as characteristics of the most successful youth development programs. We developed a measure of intensity based on frequency of program contacts and the duration of involvement with the program. Frequency was coded into three levels: less than two hours a week, between two and four hours a week, and five or more hours a week. Duration was also coded into three levels: less than a school year (e.g., one semester), at least a school year up to one full calendar year, and multiyear programs. Using these two variables we created a nine-category intensity measure and were able to classify 30 of the 45 programs reviewed, based on program documents and site visits. Results were:

- 5 high-intensity programs (17 percent), offering more than a full year of programming with at least two hours a week of program contact per youth;

- 13 medium-intensity programs (43 percent), of which 3 offered less than a school year but five or more hours of weekly programming, and 10 offered a full calendar year with two to four contact hours a week;

\footnotetext{
${ }^{1}$ The inability to identify positive youth development objectives for these programs may not be a result of the quality of the interventions; instead, the AFL grantee may have designed an intervention that captures the ideas of one or several objectives but the program documents may not convey this effort. It may also be that programs choose to focus on one or two objectives instead of attempting to achieve many objectives through one program.
} 
- 12 low-intensity programs (40 percent), of which 10 offered less than two hours a week for less than a school year (usually, for one semester, but some were even shorter).

\section{Challenges Facing AFL GRANTEeS}

AFL grantees reported a number of challenges to running successful programs. Thirty-one percent (12 programs) were having trouble recruiting or retaining youth participants. A similar number had staffing difficulties, involving high staff turnover, not enough staff, and staff without adequate training to do the job. Twenty-three percent (9 programs) reported difficulties involving parents or other family members. Twenty-one percent ( 8 programs) were having trouble forming or stabilizing relationships with schools or other organizations whose cooperation they needed for their program to work as designed. Thir ty-three percent (13 programs) had problems related to evaluation, which will be discussed in much greater detail below. And seven programs reported other challenges including fundraising and getting to the right level of program intensity for their youth.

\section{PROGRAMMATIC FINDINGS FROM THE SITE VISITS}

Site visits were undertaken for two reasons: to understand how YD is being used to enhance abstinence messages and how abstinence and YD themes are being integrated, and to determine whether it would be possible to create and evaluation design that could assess the independent effects of YD and abstinence program components.

Programs for site visits were selected using three criteria. They had to have major YD components, be stable and functioning at full capacity (i.e., not in their first year), and have a significant, reasonably sophisticated evaluation effort in place. In addition they had to be on the East Coast for budgetary reasons. The seven programs visited, arrayed from north to south, are:

1. Peer Leader Program (PLP), People's Regional Opportunity Program—Portland, Maine;

2. The Real Deal, Be'er Hagolah Institutes-Brooklyn, New York;

3. Leadership 101, Crozer-Chester Medical Center-Chester, Pennsylvania;

4. Teaching Responsible Actions in Life (TRAIL), Public Health Authority of Cabarrus County-Kannapolis, North Carolina;

5. The Children's Council—Lancaster, South Carolina;

6. Family Action Model for Empowerment (FAME), BETA Center-Orlando, Florida; and

7. Fifth Ward Enrichment Program—Houston, Texas.

The research team summarized findings with respect to six themes: auspices, whether abstinence or YD emphasis came first in a program's history, the level of integration of YD and abstinence activities, the curricula used, the length of youth involvement, and the program's main collaborative partners.

- Auspices. Collectively, these programs show that many different types of agencies can successfully mount YD and abstinence programming. Each of the seven operates under 
different auspices, including a community action agency, a public health authority, a hospital, and a family services center.

- Which came first. For five of the seven programs, their youth development focus preceded abstinence education by many years. Two of these five had a teen pregnancy prevention/sexuality focus during those years, but approached their task largely through future-oriented youth development activities.

- Integration of YD and abstinence activities. In all seven programs the youth development and abstinence activities are so integrated that it would impossible for an evaluation to determine their separate effects. Even in the four programs with an identifiable time period devoted to abstinence education, the approaches used to convey the abstinence message are YD approaches.

- Curricula. Not surprisingly, given the very highly integrated YD and abstinence approaches of these seven programs, even the ones that use standard curricula and try to achieve fidelity are faithful mostly to the messages, not to the curriculum on paper. They are all trying not to feel like school, and the way they accomplish that is to avoid approaches that stress books, lessons, paper exercises, and similar school-like components.

- Length of youth involvement. Five of the seven programs encourage youth involvement over several years-some as long as six or seven years for youth first recruited during middle school. One program targets only 9th graders, for one school year. The remaining program is very short—only 12 weeks.

- Collaborative partners. Schools are primary collaborative partners for six of the seven programs. One program is a (private) school, but still has major interactions with its local public school system. For the others, schools serve as major points of contact with youth, and in some instances also as the venue for activities. One program is based in public housing projects and has significant collaborative interactions with the local department of health. Communities in Schools plays a big role in two sites.

\section{FINDINGS FROM REVIEWS OF AFL GRANTEE EVALUATIONS}

Reviews examined evaluation design, measures, and challenges. Of the 45 AFL grantees whose documents we reviewed, 2 reported random assignment control groups, 16 (including 4 of those visited) reported comparison group designs, 11 (including 3 of those visited) compared pre- to posttest results but had no comparison group, 8 did less than pre- and posttests for evaluation, and 8 did not provide enough information to judge.

With respect to sexual behavior, 5 measured only knowledge and attitudes; 1 (a site visit program) measured attitudes and behavior; 18 measured knowledge, attitudes, and behavioral intentions (including 3 of the site-visited programs); and 14 (including 2 site-visited programs) 
measured knowledge, attitudes, intentions, and actual behavior. Inadequate information was available to classify nine programs, including one of the site-visited programs.

Most programs collect data during the first week or two of program participation each year (pretest data), and again during the last day or two of program participation, which may be as short as 5 weeks later or at the end of the school or calendar year. Very few programs do any additional follow-up, nor do any of the multiyear programs analyze their data from year to year to assess the persistence of program effects. Many programs appear to focus almost exclusively on outcome measures related to sexual behavior, although some expand their outcome measures to indicators of other risky behavior such as alcohol or drug use. Some programs include selfreported school performance (i.e., grades) on their posttest questionnaires, but none, to our knowledge, get actual grades from school systems despite strong collaborative relationships in many instances. Most of the site- visited programs also measure youth development objectives, of which self-esteem, self-confidence, and self-efficacy are the most commonly measured.

\section{RECOMMENDATIONS}

Based on study findings, we can draw several conclusions about how OAPP might proceed to strengthen its evaluation activities. We classify these into three groups-strengthening the usefulness of year-end reports, of individual program evaluations, and of cross-program evaluations.

\section{STRENGTHENING THE USEFULNESS OF YEAR-END R EPORTS}

Several changes in AFL grantee reporting requirements could give OAPP much better information from the reports it routinely receives from grantees.

\section{Revise the reporting requirements and format of the AFL grantees' year-end report to require all grantees to use a similar detailed structure and report certain basic information in a similar fashion.}

The breadth, depth, and overall quality of the year-end reports varied significantly across the AFL grantees. OAPP could be learning significantly more from these documents if they had a consistent format and reliably contained a minimum set of consistent information.

\section{Require programs and evaluators to record and report information about program intensity and average "dosage" received by participants.}

One of the more striking omissions from most year-end and evaluation reports was the lack of information on participation levels. Participation rates and dosage information are important in understanding whether the program is actually serving the children it intends to serve at the intensity for which the program was designed. In addition to requiring this information in its reports, OAPP should determine the participation statistic or statistics that would be most useful for its purposes, and then specify that this is what it wants to receive. Creating a uniform participation measure or series of measures will give OAPP another standardized way of monitoring the progress of its grantees. 


\section{Routinely summarize the year-end reports for internal use in assessing which programs incorporate the abstinence education and youth development practices for which evidence of effectiveness exists.}

Once reports contain relevant information in a consistent format, OAPP's evaluation staff and project officers should conduct their own annual summary of grantee activities, covering what each program is actually delivering to youth; classifying program activities by program intensity, dosage, and content; identifying use of evidence-based practices; and beginning to set up a structure for comparing across programs. This review would also be an essential first step in structuring future evaluations to learn more about how youth development activities could enhance the effectiveness of abstinence education programs, which we discuss further below.

\section{Develop a core set of outcome measures that each program is required to collect.}

To be able to assess the relative effectiveness of different AFL projects, it is essential that each project measure certain outcomes and measure them consistently. We understand that OAPP is well advanced in developing such a core set of measures and gaining clearance to use them. We think this will be an important step forward in helping OAPP learn the most from the AFL demonstration programs.

\section{Strengthening Individual Program Evaluations}

The evaluation designs pursued by the seven site-visited programs tend to be among the stronger evaluations being done by AFL grantees, as a relatively strong evaluation component was one of the criteria for selecting the sites to visit. Even these evaluations could be improved. Generalizing from aspects of the designs as currently being conducted plus suggested improvements, we developed some recommendations to OAPP to strengthen the evaluation information it receives from individual program evaluations.

\section{Encourage enhancements to grantee evaluations.}

Some evaluations lacked a clear logic model. While most evaluations were explicit about how they measured program objectives, what they did not discuss was the theoretical link between the intervention components and the stated objectives. Articulating these linkages better and designing evaluations to test them will assist the grantees and OAPP staff in understanding why certain program components are effective or not.

A major issue with evaluations was the relatively small number of programs using comparison groups in their design. Fewer than half of the 45 grantees programs reviewed had evaluations that used a comparison group design — a ratio that only improved slightly among the programs site visited (4 of 7 programs, or 57 percent, had comparison groups).

AFL grantees should also be encouraged to measure outcomes beyond sexual behavior, and to use OAPP's standardized measures to do so (see recommendation 4, above). It is also important to note that these recommendations may be difficult to achieve given AFL grantees' limited 
resources for program evaluation. OAPP may want to enhance the evaluation resources for programs that commit to conducting more sophisticated designs, and that offer interventions using evidence-based practices known to reduce risky sexual and other behaviors and to achieve youth development objectives.

\section{Provide technical assistance to grantees wishing to enhance the positive youth development strategies they employ and/or the strength of their evaluations.}

The excellent programs we visited were doing well with youth development strategies and had much they could teach other AFL grantees that wanted to develop or enhance a YD orientation. As we found ways to strengthen the evaluation designs even of the programs we visited, it is clear that many AFL grantees could use help with conducting more effective evaluations.

\section{STRENGTHENING OAPP's ABILITY TO ASSESS EFFECTIVENESS ACROSS GRANTEES}

OAPP could learn a good deal more for its evaluation dollar if it did one or more cross-program evaluations using consistent measurement and program design. The options recommended below could be pursued separately, but the strongest approach would be to do the first, then use all the rest in a systematic cross-program evalua tion conducted in addition to individual grantee efforts.

\section{Perform a Meta-Analysis of Existing Evaluations}

A meta-analysis of findings from existing AFL grantee evaluations should extract two types of information from year-end and evaluation reports or, if necessary, from calling programs: (1) program descriptions, and (2) evaluation outcomes.

For program descriptions, one could use the program characteristics identified by the present research (i.e., duration, frequency, presence of YD components). Extracting evaluation outcomes will require several steps to clarify which outcomes are measured, which measures were used, and which might be considered comparable across programs. The meta-analyst will have to identify the variables for which sufficient data exist to perform cross-program analyses, and for which measures are sufficiently similar to make such analyses defensible. The cross-program results will give OAPP a useful handle on some of its most commonly measured outcomes, and also identify the major gaps in information that a good cross-site evaluation should seek to fill.

\section{Conduct a Major Cross-Program Evaluation}

Given that the AFL grantees with the most YD content have program approaches that do not lend themselves to separating the effects of abstinence education and YD strategies, we sought alternative evaluation designs OAPP might pursue with its existing and potential future grantees. We think the best alternative is to compare AFL grantee programs to each other, first stratifying the programs by their intensity and YD content/approach. We suggest several possible categories, but the final ones will have to depend on the actual configurations of grantee programs. 
1. No YD, low intensity-Grantees with very little or no YD component, whose interventions are classroom only and of relatively low intensity (i.e., one semester or less);

2. No YD, medium intensity-Grantees with very little or no YD component, but whose interventions include some component that goes beyond school classrooms and also lasts at least a whole school year;

3. Some YD, medium intensity-Grantees that combine YD and abstinence, but are short, or don't meet very often, or are classroom only, or some combination of these traits;

4. Lots of YD and high intensity_-Grantees, such as many of those we visited, with major YD components and high intensity.

OAPP might select some programs from each group and develop a consistent evaluation design across sites. The design developed should follow many of the suggestions given above for strengthening individual program evaluations, including reasonable comparison groups, measures of dosage by the variety of ways that content is conveyed and by the specific types of content offered, and inclusion of a broader array of outcome variables (e.g., school performance, abstinence from many types of risky behaviors, relationships with parents and teachers).

The evaluation should take particular pains to measure both the content and mechanisms that programs use to deliver abstinence messages, and do the same for activities promoting YD objectives. Analyses should include multivariate methods to help sort out the effects of programs with a complex array of components on a range of dependent variables. Finally, as new grants are awarded, OAPP might consider how to help them incorporate data collection and evaluation that will provide good data for decisionmaking.

\section{The Cross-Program Evaluation Should Include Assessments of How Dosage and Client Difficulty Affect Results}

It will be very important to include in any cros s-program evaluation design the collection and analysis of variables describing the youth participants of AFL programs. The first of these is client participation levels ("dosage"). All available evidence indicates that longer, more intensive, and more comprehensive programs have longer-lasting effects. OAPP would want to be able to compare its results to those of previous studies to show how its programs fit into the full array of youth-serving programs.

"Client difficulty" is another aspect of youth participants that it is most important to measure and include in analyses. Programs that take only very "easy" youth-those from relatively problemfree neighborhoods and with relatively supportive families-are likely to show better results with less effort than programs that take youth with more problems, or coming from more troubled neighborhoods that expose youth to more opportunities to engage in risky behavior. A crossprogram evaluation should collect information about client, family, and neighborhood characteristics that could affect the ease or difficulty of obtaining desirable program outcomes. Some subset of these variables will be important to include in impact analyses. 


\section{Include Follow-Up Assessments of Impact}

The strongest programs should impact not only the short-term sexual behaviors and intentions of adolescents, but also should send them on a trajectory that minimizes risk-taking behavior in the long term. Given the need for programs that affect children for the long term and potential for "fade-out" effects, OAPP should encourage evaluators to conduct follow- up studies of program participants.

- For the least intense programs (e.g., one-semester programs), these follow-ups should occur at least one full semester after participation ends, and one full year would be better. If the effects cannot endure that long, perhaps the programs are not worth funding.

- For programs in which youth participate for more than one year, analyses should be conducted to see whether effects persist and intensify from year to year.

- For the recommended cross-program evaluation, both longer-term follow-up and year-toyear analyses should be conducted.

\section{Conduct Cost-Effectiveness Analyses Against Desirable Program Outcomes}

Government-funded programs are increasingly being asked to justify their costs by showing what their grantees are producing for the funds being invested in them. This is a very reasonable expectation, but too often it is demanded without thinking of what will provide the most meaningful indication of "value for money." To our minds, the only appropriate costeffectiveness indicator is "cost per desired outcome achieved." All too often, however, the only cost measures available, or calculated, are "cost per participant."

The problem with "cost per participant" measures is that a program can be very cheap per participant, but accomplish nothing. In that case all the money invested in the program is wasted from the perspective of achieving program goals. The goals, after all, are not just to serve people, but to change them, to move them in the direction the program sponsors want them to go.

The need to defend program expenditures by showing that the investment is producing good outcomes means that OAPP should build a "cost per desirable outcome" cost-effectiveness analysis into the cross-program evaluation that is the major recommendation of this report. 


\section{CHAPTER 1}

\section{INTRODUCTION AND OVERVIEW OF THE REPORT}

\section{BACKGROUND}

Adolescent premarital pregnancy and childbearing remain major social, health, and economic problems in the U.S., even as birthrates to teenagers have fallen during the last 11 years to a new record low (Sonenstein et al. 2004). Birth rates among teenagers were down in 2002, continuing a decline that began in 1991. The birth rate fell to 43 births per 1,000 girls 15-19 years of age in 2002, a 5 percent decline from 2001 and a 28 percent decline from 1990. The decline in the birth rate for younger teens, 15-17 years of age, is even more substantial, dropping 38 percent from 1990 to 2002 compared with a drop of 18 percent for teens 18-19 years. Nonetheless, the U.S. teen birthrate is still one of the highest among developed countries, generating considerable public concern about the social and financial costs of early childbearing in this country.

In fact, as part of a study funded by the Office of Population Affairs (OPA) and focusing on "Future Directions in Family Planning Research" (Sonenstein et al. 2004, p.37), a panel of experts reiterated this concern by noting that identification of successful approaches to address the sexual and reproductive needs of adolescents is a high priority. Specifically, the panel posed the following questions: "How can adolescents be better connected to their families and schools, and will these connections result in decreased sexual activity?" and "What effective practices can [we] use to assist adolescents and young adults in sexual decisionmaking?"

Youth development (YD) strategies, most likely in conjunction with appropriate age-graded sexuality and family life education programs/curricula, may have an important role to play in formulating convincing answers to these questions. Youth development approaches place considerable emphasis on helping youth develop their assets, rather than concentrating on their difficulties. They focus on where youth are going, helping them develop a belief in a viable future and in their ability to take actions that will bring that future about. The commitment to a future that would be disrupted by a pregnancy during adolescence is about the only thing that Zabin and her colleagues (1986) found to differentiate among Baltimore adolescents using teen clinics who did and did not get pregnant. Teens without a strong reason to avoid pregnancy got pregnant at the same rate as those who wanted to get pregnant; the only teens who were successful at avoiding pregnancy were those who had a future goal that a pregnancy would disrupt. Thus, incorporating youth development principles along with some specific techniques into the work of OPA's abstinence-oriented programs would seem to be an important program enhancement with potentially valuable effects.

While the trend lines for teen pregnancy and birth rates continue declining, the explanations for the decreases are many, varied, and likely to be operating in complex confluence. Clearly, health, social, interpersonal, economic, and educational factors operate simultaneously and interactively. For example, increased concern about HIV and other sexually transmitted diseases (STDs), increased concern about the negative consequences of too-early childbearing, increased interest and programs to move families and individuals from welfare to work, emphasis on taking 
personal responsibility for one's own behavior, and a renewed focus on holistic and positive approaches to youth development have all contributed to widening the scope of early sexuality education to include, and sometimes to focus exclusively on, abstinence-only approaches to primary prevention of early and risky sexual behaviors.

At the same time, there is an ever-strengthening impetus throughout government and among prevention scientists toward performance measurement and use of strong scientific evidence to aid decisions about funding, implementation, and extension of social, health, and educational intervention programs and services. This emphasis has led to an increasing desire to conduct rigorous outcome and impact evaluations, although the funding to support adequate evaluation designs has usually been lacking. In the area of sexuality and family life education-especially classroom-based programs-we are still early in the process of building a strong evidence base. A small number of evaluations of comprehensive programs (denotated "Abstinence Plus" curricula) that appear to be relatively effective in postponing first intercourse, decreasing the number of partners, increasing contraceptive use, and/or decreasing the incidence of pregnancy, births, and STDs are based on strong experimental or quasi-experimental designs (reviews by Kirby 2001; Manlove, Franzetta, et al. 2004; Manlove, Romano-Papillo, \& Ikramullah 2004). Currently, the evidence base for abstinence-only programs is more equivocal—primarily because these programs are newer, rigorous evaluations have only recently been fielded (e.g., ASPE's abstinence-only experiment conducted by Mathematica Policy Research and the University of Pennsylvania). Further, many of the most promising programs and approaches target preadolescents, in an attempt to reach teens before they initiate sexual activity and while their attitudes and intentions may be more amenable to influence. This age targeting is appropriate, given what we know about many interventions (captured in the phrase "too little, too late"). But from the perspective of trying to evaluate the impact of these programs, the short span of most follow-up data collection schedules (i.e., 6-24 months postintervention) will not capture the times when these participants begin to experience dating and sexual risk-taking situations.

\section{RESEARCH QUESTIONS AND METHODS}

In the face of compelling evidence that teen birth rates declined during the same period that implementation of abstinence-only programs/curricula and youth development/developmental assets approaches proliferated, it is sensible to examine systematically the documented relationships among provision of abstinence education together with YD programming; these may affect developments and changes in teen sexual risk-taking behaviors as well as pregnancy prevention. The research reported here is intended to be a strong first step in the investigative process. Specifically, it sought to answer to the following questions:

4. What can be learned from a comprehensive review of the relationships identified in research between YD activities and prevention of sexual risk taking?

5. What additional information can be learned from a careful and systematic review of the year-end reports of current AFL grantees who are using YD activities in their intervention programs, augmented by input from OPA project officers?

6. What additional information, insights, and empirical relationships might be learned about adherence to program models and materials (i.e., program fidelity) and sexuality- 
related outcomes for the combined YD and abstinence education approaches delivered in the most "evaluable" AFL sites?

The project had as its ultimate aim helping OPA by answering these questions in a way that provided strong guidance to the next generations of AFL grantees. Issues include the importance/salience of including specific YD program concepts, elements, and components in intervention programs and services to increase the synergy with and potential effectiveness of abstinence education programs; and ways to evaluate these enhanced programs to show the effects of augmenting abstinence education with YD approaches.

We used three different methods to answer the research questions.

- Literature review. We reviewed all recent literature pertaining to abstinence or delay of sexual activity, with special attention to how factors that are the target of youth development approaches have been shown to affect these outcomes.

- Document review. We reviewed the year-end reports and evaluation reports for all AFLfunded projects that had submitted at least one such report by December 2004.

- Site visits. On the basis of document reviews and AFL project officer advice, we selected seven AFL grantees that appeared to be using YD approaches extensively, and visited them to learn in detail how they structure their programs to include both abstinence education and strategies to promote youth development.

\section{THE REST OF THIS REPORT}

Chapter 2 presents the results of the literature review. It describes findings from numerous research and evaluation studies pertaining to two questions. The first question is: "What YD strategies are most effective at increasing commitment to abstinence or delay of sexual onset?" as relatively few evaluations contain the information needed to establish this direct linkage between YD strategies and abstinence goals, we also sought to answer a second question: "What YD strategies are effective at promoting YD objectives (e.g., increasing bonding, increasing educational competence) that are themselves expected to increase commitment to abstinence or delay of sexual onset?"

Chapter 3 presents the results of the document review, summarizing findings on approaches to abstinence promotion, YD activities in AFL-funded projects, challenges in implementing the project as planned, and evaluation strategies.

Chapter 4 presents the results of site visits. It offers brief summaries of the seven projects visited, discusses the degree to which YD activities and abstinence-oriented activities are integrated, and whether the two program components might be separated for evaluation.

Chapter 5 concludes the report with recommendations for how to improve evaluation designs to document the effectiveness of AFL grantees. It covers using year-end and evaluation reports to better advantage, improving the evaluations being conducted by individual programs, and 
strengthening OAPP's ability to learn more about the effectiveness of its programs in general and the ways that YD strategies may enhance abstinence messages in particular. 


\section{CHAPTER 2}

\section{REVIEW OF RESEARCH ON EFFECTIVENESS OF INTERVENTIONS TO PROMOTE ABSTINENCE, WITH SPECIAL ATTENTION TO THE ROLE OF YOUTH DEVELOPMENT ACTIVITIES}

Over the last five years, numerous journal articles and research reports have attempted to synthesize the large body of evaluation research dedicated to preventing premarital sexual activity and adolescent pregnancy. These reviews have been quite ambitious, using both metaanalytic and narrative reviews to systematically examine the evaluation results of pregnancy prevention interventions ranging from sex education to service learning. In general, these reviews have been helpful in advancing the overall understanding of what kinds of programs work to postpone first sexual intercourse and to reduce pregnancy among adolescents.

While these reviews often draw conclusions concerning which individual programs are effective (e.g., Manlove, Franzetta, et al. 2004; Manlove, Romano-Papillo, and Ikramullah 2004) and whether specific prevention strategies can work to achieve the desired outcomes (e.g., DiCenso et al. 2002), one drawback of these efforts is that the common components, elements, or activities shared by successful programs often are not clearly articulated. Therefore, these reviews are not as helpful for those wishing to understand whether a specific program component (e.g., classroom curriculum) or activity (e.g., role playing and guided practice) can be effective in achieving outcomes related to the postponement of first intercourse and the prevention of adolescent pregnancy.

This drawback has important implications for the Office of Adolescent Pregnancy Prevention (OAPP). While OAPP encourages the use of youth development (YD) strategies among its Adolescent Family Life (AFL) grantees, the office currently does not have adequate information on the types of YD strategies (e.g., exercises to promote parent-child connectedness) that are most effective in giving youth the means and motivation to avoid sexual risk-taking behaviors. While YD programs are often part of the major literature reviews, these reviews have not focused in depth on the YD strategies that may work to delay sexual activity or reduce adolescent pregnancy.

This paper, therefore, reviews literature pertaining specifically to the effectiveness of YD strategies in helping to prevent adolescent premarital sexual activity and pregnancy. The review has two purposes. First, it will identify, to the extent possible, evaluations that suggest (statistically) significant relationships between specific YD approaches and the delay of first sexual intercourse and pregnancy prevention. Unfortunately, because YD programs often do not have the explicit goal of postponing first sexual intercourse or preventing adolescent pregnancy, their impact on these outcomes is often not measured or is measured poorly/imprecisely. Therefore, in addition, the review will also identify evaluation studies and discuss research findings concerning the effects of selected YD strategies on some salient antecedents associated with early sexual intercourse and adolescent pregnancy. Given that these antecedents are well 
researched and documented, understanding how YD strategies may affect them is important to understanding how these approaches may help to delay the onset of sexual activity.

We begin by discussing the methods used for this review. Next we discuss findings concerning the impact of YD activities on delaying first sexual intercourse and preventing adolescent pregnancy, as well as the antecedents related to early onset of first sex. We conclude with a discussion of some lessons learned from the research, including the common YD strategies that are used by programs that have been effective in preventing premarital sexual activity and adolescent pregnancy, as well as those that have had a positive effect on the antecedents to early sexual intercourse.

\section{LITERATURE REVIEW METHODOLOGY}

To ensure that the relevant literature was examined for this review, we employed a two-stage literature search strategy. First, recent large-scale reviews and meta-analyses of positive YD and adolescent pregnancy prevention programs were examined. These reviews included:

- Not Yet: Programs to Delay First Sex Among Teens (Manlove, Romano-Papillo, \& Ikramullah 2004)

- A Good Time: After-School Programs to Reduce Adolescent Pregnancy (Manlove, Franzetta, et al. 2004)

- Community Programs to Promote Positive Youth Development (National Research Council and Institute of Medicine [NRC/IOM] 2002)

- The meta-analytic study, "Interventions to Reduce Unintended Pregnancies Among Adolescents: A Systematic Review of Randomized Controlled Trials" (DiCenso et al. 2002); and

- Emerging Answers: Research Findings on Programs to Reduce Adolescent Pregnancy (Kirby 2001).

From these documents, we identified programs that contained positive YD components and that were evaluated for their impact on either delaying the onset of first sex or preventing adolescent pregnancy. Only programs that were evaluated using a relatively rigorous design — either experimental or quasi-experimental-were included. Two categories of programs fell into this group: those that coupled positive YD activities with a sex education curriculum; and those that contained one or more YD activities without a sex education component but were still evaluated for their effectiveness on measures of sexual risk-taking behavior (often among other problem behaviors).

In addition, to ensure that important evaluations were not missed, especially those that were conducted in years since the above reviews were published, the second stage of the search process involved database and hand searches. The web and four specific databases were searched:

- EBSCOHost: a collection of four web-based databases (a psychological and behavioral sciences collection; a sociological collection; EconLit; and the health business full-text 
elite). These databases contain more than 1,000 journals representing psychology, social and behavioral sciences, health, and economics.

- Education Resources Information Center (ERIC): a database of journal and nonjournal education literature that contains 1.1 million citations going back to 1966 .

- Population Information Online (POPLINE) : a database on reproductive health that provides more than 300,000 citations with abstracts to scientific articles, reports, books, and unpublished reports in the field of population, family planning, and related health issues.

- MEDLINE: a database of life sciences and biomedical bibliographic information that contains nearly 11 million records from more than 7,300 different publications since 1965.

Each database was searched with the following keywords:

- (pregnancy OR pregnant) AND (evaluation OR adolescent OR teen OR prevention);

- (sex education) AND (evaluation OR adolescent OR teen OR prevention);

- (sex) AND (evaluation OR adolescent OR teen OR prevention);

- (abstinence) AND (evaluation OR adolescent OR teen OR prevention);

- (clinic) AND (evaluation OR adolescent OR teen OR prevention);

- (HIV) AND (evaluation OR adolescent OR teen OR prevention); and

- (HIV/AIDS) AND (evaluation OR adolescent OR teen OR prevention).

The keywords above intentionally allowed for a broad search of the literature and yielded a number of published articles and published and unpublished reports. Among these, evaluation and research articles were identified that were consistent with the goal of tracking the relationship between YD approaches and either the delay of first sexual intercourse, the prevention of adolescent pregnancy, or the antecedents to sexual risk-taking behavior.

Finally, the table of contents in a small set of important journals was also searched. These journals include:

- Journal of Adolescent Health;

- Journal of Marriage and the Family;

- Perspectives on Sex and Reproductive Health (Formerly Family Planning Perspectives); and

- Journal of Early Adolescence.

\section{UNDERSTANDING THE FINDINGS}

The reader should keep two points in mind when considering the information presented here. First, we are limited by the evaluations of YD and YD/sex education programs that have been conducted. While there may be a number of programs throughout the country that are successful 
in using YD strategies to delay first sex, we can only assess programs that have been rigorously evaluated with findings presented in journals or retrievable reports. In addition, it is important to note that in some cases specific program elements that have been evaluated may no longer reflect the "typical practice" of programs as they are currently being implemented (Manlove, Franzetta, et al. 2004; Manlove, Romano-Papillo, \& Ikramullah 2004).

\section{FINDINGS-PREVENTING PREMARITAL SEXUAL ACTIVITY}

The logic model developed for this project depicted the various ways that YD strategies might work in conjunction with abstinence education to prevent premarital sexual activity and teenage pregnancy (figure 2.1). YD activities may directly influence (i.e., reduce) sexual risk-taking behavior among adolescents by giving them the opportunity to engage in meaningful activities that make having sex and risking pregnancy less attractive. YD strategies can also affect a number of antecedents of sexual risk-taking behavior, which in turn work to delay the onset of first sex. The first section of findings focuses on the topic of primary interest to the Office of Population Affairs (bolded in figure 1)-what YD strategies are known to have a direct effect on preventing premarital sexual activity and other sexual risk-taking behavior?

Thorough evaluations of a few programs have found evidence that one or more YD strategies are able to reduce premarital sexual activity and other sexual risk-taking behaviors. In order to be included in this section, the programs and their evaluations had to possess three characteristics: (1) a strong evaluation design; (2) specific and discernable YD components; and (3) measured outcomes associated with sexual risk-taking behaviors. We describe these programs here and also summarize them in the literature review matrix (see appendix A, part 1).

A logical comprehensive approach to evaluating prevention programs is to focus on the various domains or intervention levels (e.g., families, schools, communities, peers) that likely contribute to the desired outcomes. Effective interventions to reduce sexual risk-taking behavior and to promote abstinence adequately address these domains through the implementation of developmentally based strategies. To successfully assess these outcomes related to YD activities, programs should exhibit the following characteristics: (1) be clear about the linkages between a program's interventions and specific outcomes; (2) employ a rigorous evaluation design (i.e., experimental or quasi-experimental) that allows for (a) pre- and posttesting and a follow-up of the participants, and (b) structured control or comparison group so that effects on the treatment group can be clearly linked to the interventions or other programmatic strategies; (3) interpret evaluation results consistent with the limits of the evaluation design; and (4) use evaluation instruments that reliably and validly measure the level of program participation, the intensity of the interventions, and the desired outcomes. 
Figure 2.1: Logic Model Depicting Theoretical Impact of Youth Development Strategies on Sexual Risk-Taking Behaviors (bolded boxes and arrows)

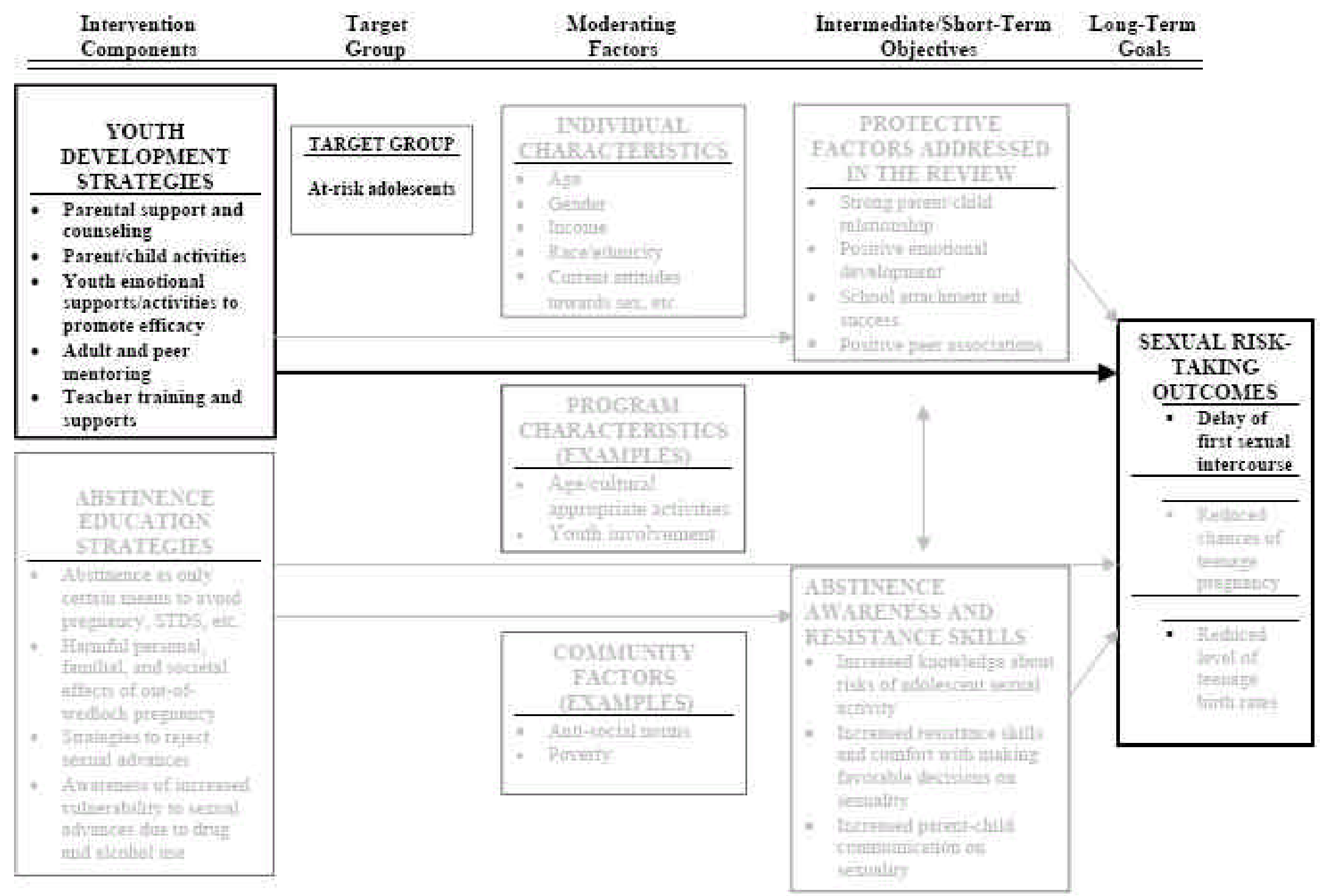




\section{The Programs Demonstrating Positive EfFects}

The programs described in this section provide a reasonably comprehensive snapshot of seven relatively well-documented programs designed to reduce or prevent sexual risk-taking behavior through various interventions that address one or more social domains. ${ }^{2}$ These programs generally show positive results, suggesting that those who receive the intervention(s) are more likely to increase their probability of remaining abstinent. These studies have several common elements, including:

- one or more social domains addressed through various interventions;

- substantial number of participants in both the treatment and comparison or control groups;

- YD activities (e.g., community service learning, parent-child communication workshops);

- pre- and posttest of participants to assess short-term changes;

- longitud inal or follow- up testing of participants to assess longer-term effects; and

- positive results in desired outcomes (e.g., decrease in sexual risk-taking behavior, increase in knowledge of and attitude towards problem behaviors in addition to improvement in actual behavior, increased academic achievement, improved parent-child communication, etc.)

The seven programs are clear about their intervention(s) and how they are expected to produce desired outcomes. They have the ability to measure outcomes as they relate to the distinguishable YD activities set forth in their program. The thorough descriptions of these seven programs in this section and the snapshots provided in appendix A, part 1 describe each program, its YD components or interventions, its evaluation design, and outcomes, specifically those related to sexual risk-taking behavior.

\section{Teen Outreach Program}

The Teen Outreach Program, a school-based program that involves youth in their communities, has a relatively well-documented ability to reduce problem behaviors among adolescents such as teenage pregnancy, school failure, and school dropout. ${ }^{3}$ The program links community service learning to a classroom-based curriculum that stimulates discussions on a variety of issues, from family conflict to human development. The combination of the interventions is designed to enhance participants' sense of autonomy while still maintaining a strong level of connectedness to the program staff and other adults. Participants engage in community service learning activities supervised by trained project staff in constant communication with community members. Some examples of these activities are working in nursing homes and hospitals and tutoring peers. Sites were expected to require a minimum of 20 hours per year of volunteer time from the participants. The Teen Outreach curriculum is designed to engage students in structured discussions, group exercises, and role-plays. Classroom discussions followed the lectures.

\footnotetext{
${ }^{2}$ The eight entries in the matrix reflect that there were two separate evaluations of the Teen Outreach Program.

${ }^{3}$ Outcome results are based on seven years of consecutive data involving more than 6,000 treatment and comparison students in more than 130 sites nationwide.
} 
The two most recent studies done on the Teen Outreach Program both show positive results in reducing sexual risk-taking behavior and improving academic performance. The earlier study included site-level data from 66 sites nationwide and over 2,000 treatment and comparison students from 1987 through 1992 (Allen et al. 1994). This study used a quasi-experimental design with a pre- and posttesting of all participants. Students entered the program's treatment group through a health class, elective, or after-school program. Those who entered the wellmatched comparison group did so in one of three ways: (1) students in the treatment group were asked to fill out a questionnaire in which they were to decide which classmates had similar characteristics; (2) school personnel suggested which students should be in this group; and (3) if the program was overenrolled, the remaining students would be placed in this group. The participants involved in this study ranged from 11 to 19 years old and were in 7 th through 12th grades. The majority of the participants were white and African-American, and 71 percent were girls. The average site gave its participants 33.5 hours of volunteer work apiece.

In the later study, the Teen Outreach Program was replicated in 25 sites nationwide with 695 high school students (grades 9 through 12) enrolled in the program (Allen et al. 1997). The majority of the students were African-American, and 86 percent were girls. Similar to the earlier study, the evaluation also addressed teenage pregnancy and school failure. The high school students were randomly assigned to either a treatment or control group and were given a pre-test at program entry and a posttest at program exit nine months later. The average site in this study gave its participants 45.8 hours of volunteer work apiece.

Results from both studies of the Teen Outreach Program evaluation have been consistent with prior research. Students in the treatment group reported lower chances of teenage pregnancy, school failure, and academic suspension. The program was very successful in achieving these goals when autonomy-promoting activities (e.g., independent volunteer work) were most prevalent. The earlier study found that the number of problem behaviors at program entry is a strong predictor of the number of problem behaviors at program exit (i.e., those with fewer problem behaviors at program entry had fewer problem behaviors at program exit $)(p<.01)$. The study also found that among those in the treatment group who had been pregnant prior to program entry (4.8 percent), a smaller percentage became pregnant by program exit (3.2 percent). In the later study, the percentage of students in the treatment group who became pregnant at program exit declined (from 6.1 to 4.2 percent, $p<.05$ ), and the percentage of students in the same group who failed courses or were suspended at program exit also declined $(p<.001)$.

\section{Seattle Social Development Project}

A study on the Seattle Social Development Project (SSDP) examines the effect of school bonding at different grade levels on problem behaviors (Hawkins et al. 2001). The project hypothesizes that school bonding protects against health and behavior problems and when introduced earlier in a child's development, it has a longer-lasting effect than if the intervention occurs during the later years. The model suggests that early and sustained intervention through the elementary school years places children on a more positive developmental path and results in fewer problem behaviors over a long period of time. The components of this project include the 
following: (1) training for teachers to effectively manage a classroom; (2) a social competencepromoting curriculum that encourages teacher-student interactions; (3) a developmentally sequenced parent training curriculum that addresses the importance of parental monitoring and reinforcing prosocial behavior, encouraging children to succeed in school, and helping children build resistance skills to peer pressure.

The study was based on a matched school quasi-experimental design. Three groups of students were involved in the evaluation of SSDP. The full intervention group $(n=134)$ consisted of all students in grades 1 through 4 , who were randomly assigned to different classrooms in eight elementary schools. The late intervention group $(n=243)$ consisted of 5 th and 6th grade students who received the intervention during these years. The control group $(n=206)$ included students in grades 5 and 6 who received no intervention at all and who were not in the full intervention group during their elementary years. All participant s came from low-income households in Seattle, Washington, and the majority of the participants were white.

The evaluation study of SSDP found that students in the full intervention group were significantly more bonded to school than those in the late intervention and control groups at ages 13 and 18. Even though students in the late intervention group experienced an increasing level of school bonding at age 18, it was not significantly different from the level of bonding at age 18 of those in the control group. Results also show that an increase in school bonding suggests a decrease in level of sexual activity $(p<.01)$, lower number of sexual partners $(p<.05)$, and lower chance of becoming pregnant or fathering a baby. Similar directional results are also seen for other problem behaviors, such as school failure, criminal activity, and substance use and abuse. Overall, the evaluation suggests that social development interventions through the elementary school years have a positive long-term effect on school bonding and therefore shows promise for preventing problem behaviors at age 18 .

While it is important to know what short-term goals are achieved through prevention programs, it is also critical to assess the impact of these programs on participants during a longer-term period. This will provide an understanding of how robust the effects are. SSDP is a good example of a program that effectively addresses the social domains and has proven to have robust effects through the inclusion of a longitudinal research design. Evidence shows that there is a more positive long-term effect for preventing problem behaviors such as sexual activity when interventions are given throughout the primary and secondary school years rather than in the middle of their development or none at all.

\section{Carrera Program}

The Carrera Program is a year-round after-school program established in 1984 by the Children's Aid Society, designed to increase sexuality education and prevent teenage pregnancy among high-risk adolescents in Harlem (Philliber et al. 2002). The program consists of seven components: (1) Job Club (e.g., career awareness, internships), (2) academic performance assistance (e.g., tutoring, SAT preparation), (3) comprehensive family life and sexuality education (i.e., weekly sessions of an age-appropriate curriculum), (4) art activities (e.g., music, dance, writing), (5) sport activities (i.e., individual sports such as golf and swimming), (6) mental health care (e.g., counseling, crisis intervention), and (7) medical care (e.g., annual 
comprehensive medical exam). Throughout the school year, all activities operate daily for approximately three hours each day. Many of the sites divide their students into a few groups and rotate them among the activities offered. For example, one group of students might receive the comprehensive family life and sexuality education on Tuesdays and Thursdays, while another group attends Job Club on the alternate days. Those two groups would then be reversed once each intervention has been completed.

Six agencies in New York City participated in the study. These agencies randomly assigned 600 students (100 students per site; 300 in the treatment group and 300 in the control group) to the Carrera Program. The participants were eligible to enroll in the program if they were not already enrolled in an after-school program, were between the ages of 13 and 15, and were not currently pregnant or had not been parents. The majority of the participants were black and Hispanic. Students were recruited via school outreach, flyers, or families who had been contacted about the program. The evaluation used linked data from annual surveys of the participants' characteristics and program outcomes, annual tests of knowledge of sex topics given by the evaluation staff simultaneously with the annual surveys, and monthly attendance records by program staff. Baseline data were collected from February through April 1997, and the students were tracked for three years. Of the original sample, 81 percent of the participants in all groups remained for the three-year follow-up. The average site engaged its participants for 16 hours per month.

Results show that the majority of the participants reported never having had sex prior to program enrollment. When compared to the control group, students in the treatment group had significantly greater gains in knowledge over time (22 percent versus 11 percent, $p<.001$ ). Girls in the treatment group were significantly less likely to have ever had sex than the girls in the control group (54 percent versus 66 percent, $p<.05$ ). Findings also suggest that long-term connections to the program prevent teenage pregnancy by improving the girls' sexual literacy and increasing the use of effective contraceptives. However, there were no significant effects on male participants with respect to sexual and reproductive behavior outcomes. Overall, both male and female participants had a higher chance of receiving good primary health care.

\section{Client-Centered Intervention Approach}

A client-centered program aimed at reducing teenage pregnancy was conducted in seven communities in Washington from 1995 through 1999 (McBride \& Gienapp 2000). Each of the seven sites had slightly different objectives, target populations, settings, and types of project staff. However, they generally consisted of the following intervention components: (1) education and skills building (i.e., sessions held with school counselors to discuss topics related to sexuality and risky behaviors); (2) mentorship (i.e., participants are matched with adult mentors who can provide career advice, emotional support, and other types of support; and (3) advocacy (i.e., staff provided some type of support to partnering organizations such as clinics and schools). The client-centered program is different from curriculum-based models because it addresses a wide range of issues and behaviors through the use of age-appropriate and developmentally appropriate interventions. The interventions are tailored to the needs of each site's target population. 
This Washington study involved clients who were randomly assigned to a treatment or control group (based on whether their birth date is an even or odd number). A total of 1,042 students ages 9 through 13 were served at four sites, while 690 students ages 14 through 17 were served at the remaining three sites. The majority of the participants were white and female, and all participants were in grades 7 through 12. The number of hours each participant received services varied by site, since intervention also varied by site. The younger participants in the treatment group received about 14 hours of activities per year, while the older treatment group participants received 27 hours. The control group received only two hours per year. Data were collected during the pre- and posttesting periods, and were collected only in group settings.

Results varied across the seven communities. One site may have consistently shown positive results between its treatment and control groups, yet another site may not have found that the program had any effect on its participants. One site found that students who received more hours of services per year were less likely to have sex, and another site reported that the participants in the treatment group were less likely to engage in risky sexual behavior. Another site found that students in the treatment group were less likely to engage in substance use and abuse. Overall, this client-centered program showed no effects on factors that place children and adolescents at risk of becoming pregnant, such as parent-child communication and sexual values.

\section{Reach for Health Community Youth Service Learning Program}

Reach for Health is a community service learning intervention program designed to reduce risky sexual behavior (O'Donnell et al. 1999). The program offers urban middle school students opportunities to participate in volunteer work for their community, along with a curriculum that is delivered to students in the 7th and 8th grades at the treatment schools. The curriculum focused on three key health risks (i.e., drug and alcohol use, violence, and risky sexual behavior) often encountered by urban high-risk adolescents. This curriculum was adapted from the Teenage Health Teaching Modules_one of the nation's most widely used comprehensive health curriculum - and combined with elements from other nationally recognized curricula such as Being Healthy, Michigan Model, and Contemporary Health Series.

Two large urban middle schools with a total sample of 1,061 students were involved in the evaluation of Reach for Health. Students at one school were randomly assigned by classroom to receive either the Reach for Health community youth service intervention plus the curriculum $(n$ $=225)$ or the Reach for Health classroom curriculum only $(n=222)$. Students at the second middle school served as the control group, receiving neither intervention $(n=584)$. The school sites were very similar in that they have a large and almost exclusively minority student body. Students were surveyed at baseline and at the six-month follow-up session. The curriculum consisted of 40 core lessons per year for those in grades 7 and 8, and participants spent three hours per week in a community placement, such as clinics, childcare centers, and nursing homes.

Among students who reported having had no sexual intercourse at baseline, those who received the community service learning and curriculum interventions (32 percent) were less likely to report having had any sex at the six-month follow-up compared with those who received only the curriculum intervention (38 percent) or no intervention at all (41 percent). Participants also reported experiencing less sexual activity than those in the control groups $(p<.05)$. Students in 
the 8 th grade had the greatest impact because they received the most intensive service program $(p<.03)$. Special education students seemed to be the only group who benefited from a curriculum-only intervention. Overall, those who received both interventions were less likely to reduce their chances of engaging risky sexual behavior.

Programs that are able to assess or tease out the effects of YD interventions on participants are more likely to have a stronger case for why the YD components effectively reach program goals and objectives. Reach for Health demonstrates the value of adding YD activities to a classroombased sex education curriculum. Based on the rigor of its research and evaluation design, this program illustrates that those who engaged in both activities showed significant improvement in safe sex compared with those who received the curriculum only. This suggests that the community service learning activities have a strong effect on sexual risk-taking behavior when combined with a sex education curriculum.

\section{Summer Training and Education (STEP)}

The Summer Training and Education Program (STEP) was designed to stem summer learning loss among high-risk youth who are likely to drop out of school by providing summer opportunities to low-achieving youth (Walker \& Vilella-Velez 1992). The program included four key components: (1) remedial education that focused on basic skills and the learning process (minimum of 90 hours or four half-days per week), (2) life skills and opportunities that stress responsible social and sexual decisions (one half-day per week), (3) work experience at minimum wages (five half-days per week), and (4) school year support by guidance counselors and other school personnel.

STEP was originally implemented in five demonstration sites in 1985 and then replicated in more than 100 sites nationwide in 1987. The program targeted 14- and 15-year-old students who came from low-income households, and the majority of the participants were black. In both the pilot and replication sites, these students were randomly assigned to a treatment or control group. Those who were in the treatment group were exposed to all four components of STEP, while the students in the control group were only offered a summer job. In each of the demonstration sites, there were approximately 330 total participants (165 in each of the treatment and control groups), and in the replication study, over 20,000 youth were included over the four-year period. Data were collected from posttest interviews and from school transcripts with both groups.

Results from both the demonstration and replication studies are quite similar, but this section will discuss only the results from the evaluation of the replication sites. Compared with a control group, participants in the treatment group tested a half-grade higher in math and reading by the end of the summer, reported less consumption of alcohol, and significantly increased their knowledge of contraception. Among those in the treatment group who had not had sex prior to enrolling in STEP, upon completion of the program, they reported significantly greater intention to use contraception in the future if they were to become sexually active. By the end of the fouryear study period, there had been no improvement in reducing the rate of adolescent pregnancy between treatment and control groups. 


\section{Quantum Opportunities Program}

The Quantum Opportunities Program is a year-round comprehensive youth development program designed to provide disadvantaged youth education, development, and service activities over a four-year period (Hahn, Leavitt, \& Aaron 1994). The key components of this program include (1) 250 hours of education activities such as peer tutoring and computer-assisted instruction; (2) 250 hours of development activities such as job and college preparation sessions; and (3) 250 hours of service activities such as volunteer work. Community agencies provided these services during after school hours on their premises or on school grounds if time and space were available. Each student in the program was matched with an adult mentor throughout the four years.

The Quantum Opportunities Program was launched in five communities in 1989, and 50 students in each community were randomly selected to enroll in the program or to a control group where no intervention existed. These students entered the program in 9th grade and continued until the 12th grade. About 86 percent of the participants were ethnic minorities. Data were collected from both groups through periodic questionnaires and basic skills tests, which were administered throughout the four years. Approximately 85 percent of the original sample remained by the end of the four-year period.

Evaluation results show that students who were in the treatment group were more likely to perform better in school and attend a four-year college, and less likely to become teenage parents. Many of them became volunteer tutors, counselors, and mentors six months after they completed the program. Of the five sites, the Philadelphia site had a much larger and more positive impact than the other communities. Students who received the intervention at the Philadelphia site were more likely to perform better in school and less likely to engage in risky sexual behavior. Overall, the effects of the Quantum Opportunities Program increased over time. After the first year, there was no difference between the treatment and control group. Statistically significant differences became clear by the time these students were leaving high school.

\section{FINDINGS-STRENGTHENING PROTECTIVE ASSETS}

While relatively few studies have focused on the direct relationship between YD programs and adolescent sexual behaviors, other areas of the adolescent pregnancy prevention literature can be helpful in understanding the importance of YD strategies in preventing premarital sexual activity and adolescent pregnancy. For example, a vast body of research has focused on understanding the antecedents to adolescent sexual risk taking and has uncovered a number community-, family-, and individual-level factors that are associated with the initiation of sex and other sexual risk-taking behaviors. Antecedents that have been shown to increase the likelihood of these behaviors are more commonly known as "risk" factors, while those antecedents that decrease sexual risk taking are known as "protective" factors. Because YD approaches can work to increase both the number and strength of protective factors in youth, below we discuss research findings on those factors associated with the delay of first sex. We then discuss the evaluation research that provides evidence of the impact of positive YD strategies on strengthening these protective factors. Figure 2.2 presents the linkages in the logic model that are the focus of this section. 


\section{Protective Factors that D elay First SeX}

A recent review of the pregnancy prevention literature identified over 50 protective factors associated with the increased likelihood that an adolescent will delay first sex (Kirby 2001). These protective factors range from communities with higher rates of college degrees (Billy, Brewster, \& Grady 1994; Brewster, Billy, \& Grady 1993) to the high-perceived cost of pregnancy by the adolescent (Blum, Buehring, \& Rinehart 2000). Given that many of the protective forces cited in this literature are beyond the control of community-based programs, our review focuses on four sets of protective factors that programs such as the current AFL grantees might hope to affect: ${ }^{4}$

- $\quad$ strong parent/child relationship;

- positive emotional development;

- school attachment and success; and

- positive peer associations.

The research relating to each set of these protective factors is discussed below.

\section{The Parent-Child Relationship}

Perhaps two of the most studied and influential protective factors in delaying first sexual intercourse are conservative parental attitudes toward sex and what is often described as "parentchild connectedness." Recent research indicates that parental disapproval of adolescent sexual intercourse can be a strong predictor of delayed onset of first sex, ${ }^{5}$ especially when it is

\footnotetext{
${ }^{4}$ Protective factors not included here because they are ostensibly beyond the control of the programs include community characteristics; family characteristics (family structure, parental education, employment and welfare receipt); religiosity; and biological factors. Another set of protective factors that could be influenced by communitybased organizations that are not included are those associated with the sexual beliefs, skills, and behaviors of the adolescent. These protective factors are targeted by the abstinence curricula used by the AFL grantees and do not fall under the rubric of positive youth development.

${ }^{5}$ It is important to note that while disapproval of sexual intercourse coupled with parent-child communication about sex can delay first sex, higher levels of parental communication alone have not consistently been shown to delay first sex. Two issues may be at work here - one methodological and one substantive. Methodologically, it may be that these studies have not been able to separate out the fact that parents may begin to intensify their communication about sex if they suspect that their child is about to engage or has engaged in sexual activity (Miller 2002).
} 

Figure 2.2: Logic Model Depicting Theoretical Impact of Youth Development Strategies on the Antecedents of First Sex (Bolded Boxes and Arrows)

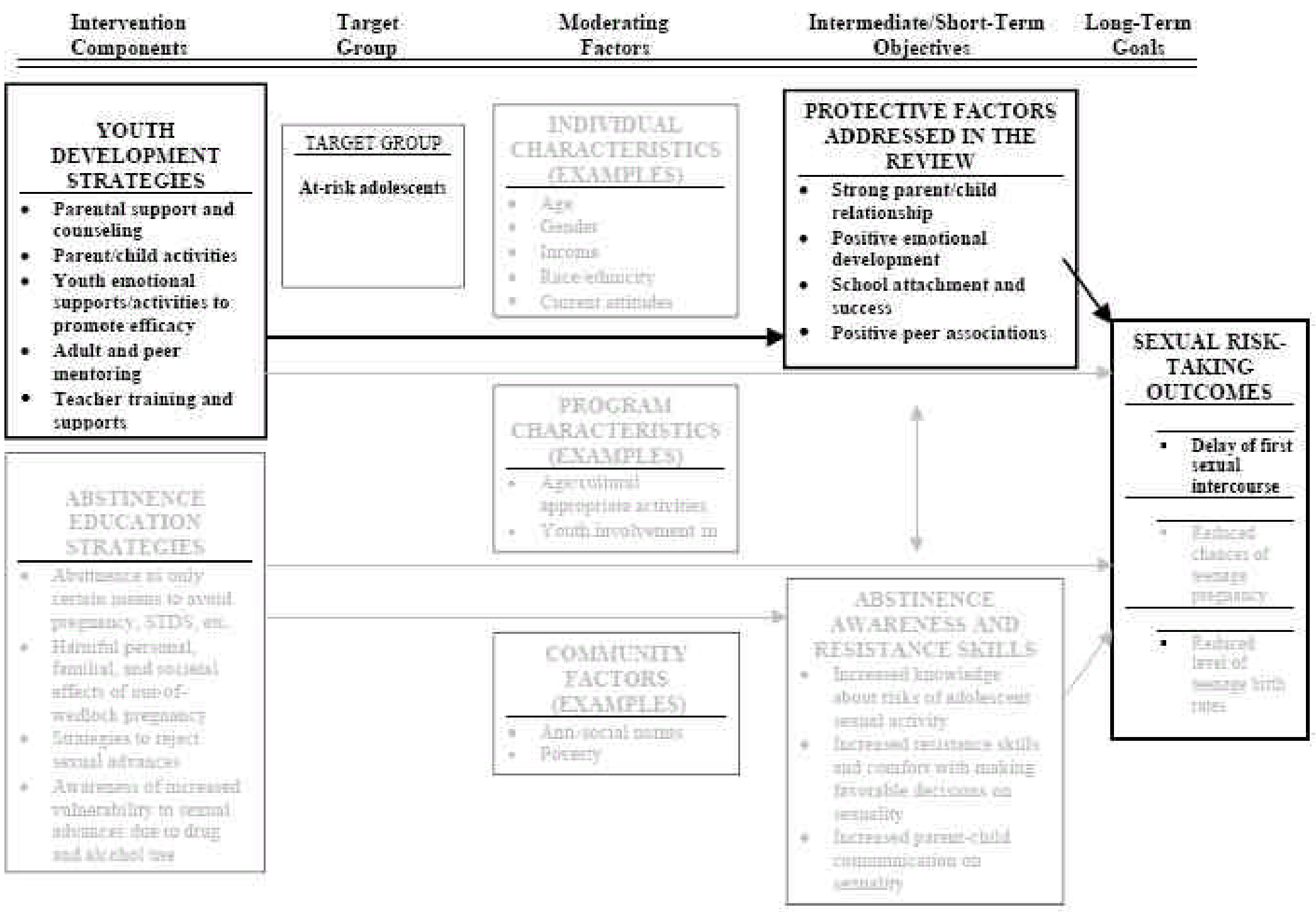


coupled with high levels of parental communication about sex (Miller 2002; Miller et al. 1997; Miller 1998). This relationship most likely occurs because parental disapproval can increase the emotional costs of sex for adolescents, making them less likely to engage in sexual behavior (Bearman \& Brucker 2001).

However, research using data from the National Longitudinal Study of Adolescent Health (Ad Health) indicates that adolescents often do not accurately perceive their parents' disapproval of adolescent sexual activity. Blum and Rinehart (2002) find that 30 percent of adolescent girls and 45 percent of adolescent boys incorrectly perceived their mother's strong disapproval of sex. Similar to past work on this issue, they find that it is the adolescent's perception of their mother's strong disapproval of sex rather that the mother's actual attitudes that is most closely associated with the delay of first sex. The strength of the parent/child relationship is important in this context because it has been found that adolescents who report being closely connected to their parents are more likely to accurately perceive their mother's disapproval compared to adolescents who are not closely connected (Sieving et al. 2000).

In addition to strengthening the influence of parental disapproval, the level of parent/child connectedness in itself can raise the costs of adolescent sexual behavior by making adolescents less willing to risk parental disappointment. Adolescents who feel close to their parents will be more affected by parental disappointment and will be more likely to abstain or postpone sexual activity. Empirically, the connectedness construct is most often measured from the adolescent perspective using a series of questions about how close adolescents feel to their parents, how much they believe their parents care about them, how warm and loving their parents are, how satisfied they are with their parents, and how good their communication is with them. In a review of about 25 studies that investigate the relationship between parental closeness and sexual risktaking activity, Miller (2002) found "markedly consistent" results that parent-child closeness is associated with less sexual risk taking, including remaining abstinent and postponing sex.

Some of the most recent work both confirms and qualifies this research. Sieving and colleagues (2000) find that the protective power of parent-child connectedness is related to the adolescent's age and gender. The authors find that while a strong mother-child relationship is related to a delay in first sex among younger girls and boys, as well as older boys, the effect of the relationship appears to diminish in older girls. Despite this finding, however, mothers in general tend to have less influence on the timing of the first sex of their sons compared to their daughters. While the effect of a connected motherchild relationship may last into middle adolescence with boys, boys are also more likely than girls to be more strongly influenced by social influences from siblings and peers (Blum \& Rinehart 2002).

\section{Positive Emotional Development}

Two aspects of emotional well-being are often discussed as being particularly important in delaying first sex: high self-esteem and an adolescent's future, aspirations, and expectations. In theory, adolescents with lower levels of self-esteem may engage in sexual activity to feel better about themselves, provide themselves with a sense of intimacy and maturity, or rebel against conventional norms (Spencer et al. 2002). Early studies of the relationship between self-esteem 
and the initiation of sex used cross-sectional data that produced conflicting and difficult-tointerpret results because these studies could not tell whether lower levels of self-esteem had moved the adolescent to initiate sex, or whether the initiation of sex had caused low self-esteem. As a result, early findings about the effects of self-esteem were mixed. One early study found that the self-esteem scores of girls who had not yet had sex were significantly higher than girls who had, while self-esteem appeared to have no relationship to the timing of sexual initiation for boys (Orr et al. 1989). Other studies, however, found no relationship between gender, selfesteem, and the initiation of sex (Blum et al. 2000; Robinson \& Frank 1994).

More recent work using longitudinal data have been able to provide evidence that lower levels of self-esteem increase the likelihood of first sex in specific cases. Interestingly, the work that has focused on both boys and girls have found that low self-esteem in girls makes them three times more likely to engage in sex, while higher levels of self-esteem in boys increased the likelihood of first sex (Spencer et al. 2002). Recent work that focused on sexually active African-American girls also found that girls with better self- concept were better at refusing unwanted, unprotected sex (Salazar et al. 2005).

Planfulness (i.e., planning for the future and future life events) is a key emotional asset for youth (NRC/IOM 2002), which is negatively associated with adolescent sexual risk-taking behavior. Some researchers have discussed that adolescents conceive of their decisions about sexual activity in terms of risks and rewards. Adolescents, given the opportunity to engage in sex, will proceed or refrain depending on how they evaluate the consequences of the behavior (Hofferth 1987). In theory, then, adolescents with strong future aspirations should be more likely to refrain from sexual activity because the perceived costs are higher. This notion has received empirical support. Resnick and colleagues (1997) found that adolescents who said they were "college bound" were less likely to initiate sex than other adolescents. In addition, commitment to future plans that would be disrupted by a pregnancy during adolescence was about the only thing that Zabin and colleagues (1986) found to differentiate among Baltimore adolescents in their sample who did and did not get pregnant. Indeed, teens without a strong reason to avoid pregnancy got pregnant at the same rate as those who wanted to get pregnant.

\section{School Attachment and Success}

Given the large number of hours that adolescents spend in school each week, it is not surprising that an adolescent's level of attachment to school can have a large effect on sexual behavior. Numerous dimensions of school attachment and performance have been shown to be associated with the delay of first sex. Researchers believe that school-related factors may help students develop hope for the future (Maddox \& Prinz 2003) and reduce an adolescent's motivation to engage in sexual risk-taking behavior (Kirby 2001). Empirical work provides evidence that school connectedness is related to a delay of first sex. Positive regard concerning how fairly teachers treat students, how close adolescents feel to people at school, and how much they feel a part of the school have a protective effect on the delay of sexual debut (Resnick et al. 1997).

Recent work has also found that academic performance is related to the timing of first sex. Halpern and colleagues (2000), for example, found that students who score high on achievement 
tests were less likely to initiate sex than students who received average scores. ${ }^{6}$ In addition, other work has found that adolescents, specifically Hispanic adolescents, were at less risk of engaging in sex if they had never repeated a grade or did not report frequent problems with school work (Blum \& Rinehart 2002).

\section{Positive Peer Associations}

As children grow older, peer activities and acceptance become increasingly important to adolescents and often take precedence over school and academic performance (NRC/IOM 2002). During adolescence, peers model behavior for each other, provide each other with social opportunities, and set social norms (Brown \& Theobald 1999). While conventional wisdom often conceives of peer influence as a risk factor for youth, research indicates that this is not always the case. In fact, it has been argued that peer influence is more often a protective than a risk factor (Bearman \& Brückner 1999). As with strong parent/child bonds, positive peer associations can increase the costs of engaging in sexual behavior.

In one of the more thorough analyses of peer effects on sexual debut, Bearman and Bruckner (1999) identify five levels of potential peer influence: (1) best friends; (2) close friends; (3) the peer group or clique; (4) the leading crowd (the most popular adolescents); and (5) the school environment. For a sample of adolescent girls from the Ad Health Survey, the authors classified each of a girl's peer groups by their risk or protective character. Low-risk peer groups were those that scored high on a number of measures of their orientation to school (e.g., GPA, attachment to school, participation in activities, etc.) and low on measures of nonnormative behavior (e.g., drinking, drug use, etc.), while high-risk peer groups scored the opposite on these two scales. While best frie nds, the leading crowd, and school context had little if any impact on the timing of first sex, low-risk close friends, particularly male close friends, protected girls from engaging in sex. Interestingly, the study also found that girls who had friends with close bonds with their parents were less likely to engage in sex, regardless of their own relationships with their parents. The study also found that adolescent girls who had friends of the same age were much less likely to initiate sex than girls with older friends.

Most work on peer influence, however, has focused on its negative effects. In their review of 15 years of research on how peers influence each other on sexual behavior, Brown and Theobald (1999) conclude that peers do have an influence, but it is not as profound as is often assumed. This has to do with the fact that adolescents often initially choose like-minded peers and that peer influences are often moderated by a number of factors, most notably quality parenting. While Brown and Theobald (1999) conclude that peer influences can be positive or negative depending on the adolescent's situation, the negative influences of peers and the importance of moderating factors comprise the major focus of the research to date. For example, the authors highlight research that has found that frequent dating, having close friends of the other sex, and having older friends are related to initiating sex at an early age (Miller et al. 1997).

\footnotetext{
${ }^{6}$ Interestingly, students who had exceptionally low scores on the test were also less likely to engage in sexual activity.
} 


\section{Two Caveats}

There are two important points to keep in mind when thinking about the protective factors discussed above. First, as Kirby (2001) points out, because so many antecedents-both risk and protective - are related to the initiation of sex, most of these factors alone are only moderately to weakly related to behavior. Therefore, as we will discuss below, it will almost certainly make most sense for programs to target multiple antecedents, especially since a number of these antecedents are highly correlated and impact each other. School performance may be related to self-esteem, which may also respond to a strong parent-child relationship.

\section{The IMPACT OF Youth Development Programs on the Protective Factors that DELAY FIRST SEX}

In this section, we review key findings from the YD literature that pertain to how YD programs can affect the antecedents of first sex. We discuss the evaluation findings from major reviews of the program evaluation literature and highlight particularly effective or compelling programs. It is important to note that the programs discussed in this section share many of the elements of the programs discussed earlier and may in fact move youth to delay or abstain from sex. We did not discuss these evaluations earlier because they did not measure the program's impact on sexual risk-taking behaviors.

\section{Two Comprehensive Reviews of Multidimensional YD Programs}

In the past three years, two major reviews have examined the findings from experimental and large-scale quasi-experimental evaluations of YD programs (Catalano et al. 2002, 2004; NRC/IOM 2002). These comprehensive reviews provide evidence that YD strategies can positively affect the four sets of antecedents discussed above. Interestingly, while much has been made of the impact of YD strategies, relatively few rigorous evaluations have measured the impact of YD strategies on these specific antecedents. This may be the case in part because of the difficultly measuring constructs such as bonding and self-esteem, but also because the YD strategies employed by most of the evaluated programs are part of a larger strategy to reduce specific risk-taking behaviors. The effect of the YD strategy on the proximate outcome (e.g., parent/bonding) is of less importance in declaring the overall success of the program than the ultimate risk-taking behavior (e.g., marijuana use).

Catalano and colleagues $(2002,2004)$ conducted a systematic review of published and unpublished evaluation literature on YD and YD programs. The research team used strict criteria for the program evaluations included in the review, including program content and evaluation methodology and identified 25 well-evaluated YD programs with at least one significant effect on a YD outcome. Of these 25, seven programs appear to address and measure one of our antecedents of interest. ${ }^{7}$ Appendix A, part 2 provides a summary of these programs. Below we summarize findings related to YD program effects on the antecedents.

\footnotetext{
7 These seven programs shared three characteristics: (1) the program addressed one of the four sets of antecedents; (2) the evaluation measured the program's impact on the antecedent; and (3) the program had a positive effect on the antecedent.
} 


\section{The Parent-Child Relationship}

A number of the 25 programs employed strategies to improve the parent-child relationship (i.e., parent-child bonding, communication, and family functioning). These programs had both schoolbased and home-based intervention components and employed one or more of the following strategies: (1) direct parent training; (2) "home engagement" activities (e.g., joint parent-child homework assignments and communication drills); or (3) parental participation in program design. Two programs employed one or more of these strategies and also measured some aspect of parent-child bonding. One program called Creating Lasting Connections, which was shown to reduce parent-child conflict, provided more intervention hours for parents than youth. Parents and children engaged in practice communication sessions and involvement in community activities, while parents also attended communication training. Another program called the Adolescent Transitions Project also had a strong emphasis on helping parents, providing a curriculum for parents that focused on prosocial fostering, limit setting, and problem solving. Both programs reduced parent-child conflict.

\section{Positive Emotional Development}

A number of the 25 programs also worked to improve a child's emotional well-being. These well-being interventions appear to be particularly prominent (and more likely to be measured) in those programs serving youth who are culturally different from mainstream youth (e.g., foreignborn and Native American youth) as well as those who have had emotionally disruptive experiences (e.g., abuse or divorce). Three programs measured emotional well-being outcomes and provide some evidence for a program's ability to improve self-concept and esteem, as well as future aspirations. The Valued Youth Partnership Program, which served a predominantly Hispanic foreign-born population, was designed to transform a child's self-concept through bilingual education, tutoring, and increased student recognition of accomplishments. The program was found to be successful in improving a child's self-concept after the first year of participation, but these findings did not endure through the second year of the program. A similar program for Native American youth called Bicultural Competence Skills provided role play and communication strategies to assist with bicultural efficacy and increase participant self-control. A third program entitled the Child Development Project improved conflict resolution skills, moved participants to exhibit more frequent altruistic behavior, and increased belief in the future as measured by educational aspirations. Finally, the Children of Divorce Intervention Program, which provided cohesion and trust building activities, worked to reduce anxiety and increase frustration tolerance.

\section{School Attachment and Success}

Activities that enhance a child's attachment to school and academic progress were some of the most common across the 25 programs examined by Catalano and colleagues $(2002,2004)$. These activities included events that brought together parents, teachers, and students; tutoring; principal and teacher education and supports; and exercises to build group cohesion and trust in the classroom. Three programs have solid measures of school attachment. One of the most noteworthy of these programs, the Child Development Project (CDP), is a comprehensive program consisting of the "Caring School Community" student curriculum, principal and teacher 
supports, school-community activities, and home-based activities. Findings from the program evaluation indicate that participants in CDP were more likely to like school, read books outside of class, have stronger academic motivation, and have a greater trust and respect for teachers. Another intensive and comprehensive program that has been shown to improve academic performance and school attachment is "Success for All," whose premise is that every child will be able to read at the end of first grade. The program provides numerous components ranging from one-on-one tutoring to professional development for teachers to a family support team. Participants in the program have shown advances in reading, especially among students in the lowest 25 percent of their grade, as well as better school attendance.

\section{Positive Peer Associations}

For the most part, the programs reviewed by Catalano and colleagues $(2002,2004)$ worked to make participants more resistant to peer influence rather than promote positive peer associations. Two programs, however, had peer bonding or acceptance components that were distinct from strengthening resistance to peer pressure. The CDP curricula, "Caring School Community," provide teachers with instruction on how to build classroom unity and provide students with skills to understand and empathize with their peers. The program evaluation found that students in the program were 10 percent more likely to have concern for their peers and 15 percent more likely to feel a sense of community at school. In addition, the Children of Divorce Program provides sessions geared to building group cohesion and trust. Program participants were found to be more sociable with their peers than control group students. While the cons tructs measured by these two programs do not perfectly capture the types of peer relationships that have been shown to delay first sex, their impact on peer bonding is notable if one believes that positive peer associations can increase the perceived costs of sexual behavior.

\section{The NRC/IOM Report}

To the findings of program evaluations, the NRC/IOM report adds a description of the common program features that make for a successful YD program. The review takes a step back from the specific YD activities that may protect against risk-taking behaviors and focuses on the general features of successful youth programs. Looking across programs that have had documented success in reducing adolescent risk-taking behaviors, the report highlights six features of these programs that, if applied, may help in improving the effectiveness of the AFL grantees. These are:

1. Structure and safety: These characteristics represent the absolute minimum requirements of a positive YD program. The NRC/IOM report notes that program descriptions rarely discuss these aspects of their programs, most likely because appropriate structure and safety represent inherent and assumed dimensions of the program. However, the report explains that the safety construct applies to both physical and psychological safety, and as such, it is important for programs to have clear and consistent rules, expectations, and boundaries in addition to a safe physical space.

2. Supportive relationships: Successful programs provide youth with regular supportive contact with nonfamilial adults. While mentoring programs have this feature as their 
fundamental goal, the adult-child relationships in any program should take the form of mentoring regardless of type or duration of contact. Major elements of supportive relationships include good communication, warmth, connectedness, caring, and responsiveness.

3. Opportunities to belong: A sense of belonging fosters health development. As youth become more independent and work to define their own identities, successful programs provide opportunities for youth to feel like they are part of a cohesive group with positive values. This point is especially true for certain groups whose home and community environments are fragmented or negative.

4. Support for efficacy of mattering: Youth who are allowed to express their points of view and are given autonomy and ways in which to feel efficacious develop more positive motivation and are more willing to engage in identity exploration. Successful youth programs, therefore, are those that give youth the opportunities to be efficacious, such as social learning approaches.

5. Positive social norms: Successful programs promote positive social norms and discourage deviant or problem behaviors. Programs promote such norms by being clear about rules of behavior and expectations for the program participants.

6. Opportunities for skill building: Successful programs work on building skills across all of the developmental domains (i.e., physical, cognitive, psychological, and social).

7. Integration of Family, school, and community: Because youth are socialized across multiple settings (community, school, and family), it is important that there be meaningful communication and synergies among them. Accordingly, successful programs integrate family, school, and community into their program design and attempt to bring together actors from three settings for meaningful involvement in the lives of youth.

\section{A Review of Mentoring Programs}

While reports by Catalano and colleagues (2002, 2004) and NRC/IOM (2002) include programs that have mentoring components, a recent meta-analysis focusing specifically on mentoring (DuBois et al. 2002) programs provides insights into the impact of mentoring on youth and the specific features that make mentoring most effective. The analysis includes evaluations that examine the impact of mentoring on such antecedents of first sex as emotional well-being, academic success, and social competence. However, the authors report the effects of mentoring on all outcomes combined, rather than on each outcome separately. Nonetheless, the findings relating to the program features that most enhance the effectiveness of mentoring may prove helpful to improve those AFL grantees with mentoring components. In order to obtain a more specific sense of how a mentoring program can affect YD, we also discuss findings from the Big Brothers Big Sisters Program evaluation. 


\section{The DuBois and Colleagues Study}

In a comprehensive review of 55 mentoring program evaluations, DuBois and colleagues (2002) assessed the impact of mentoring programs on youth. While, on average, the size of these positive impacts are modest at best, mentoring programs with specific characteristics and with a focus on certain types of youth can have much greater impacts. Indeed, at-risk youth are more likely to benefit from mentoring programs than are "average" youth, especially when a strong relationship is formed between the mentor and youth. Moreover, the strongest effects are achieved when the programs follow best practices, including:

- monitoring of program implementation;

- screening prospective mentors;

- $\quad$ systematic matching of mentors and youth based on sex, race, and common interests;

- mentor training prior to youth-mentor match;

- supervising of the mentors;

- mentor training during the youth-mentor relationship;

- a support group for mentors;

- structured activities for mentors and youth;

- a parental support or involvement component;

- expectations about the frequency of contact; and

- expectations about the length of the relationship.

The meta-analysis revealed that positive effects of programs are significantly larger when at least six of the above practices are simultaneously followed. While no individual feature or characteristic is responsible for the positive impacts, certain characteristics appear to have a more powerful impact than others. For example, programs that use mentors from "helping" roles or professions (e.g., teaching) tend to have a greater positive impact on youth than other programs. In addition, programs that provide training to mentors both before and during the their relationship with youth also seem to have a greater positive impact.

\section{The Big Brothers Big Sisters Program}

The Dubois and colleagues (2002) study helps with an overall understanding of how mentoring affects youth, but it does not help us understand the potential effects of mentoring on the specific antecedents of first sex. The random assignment study of the Big Brothers Big Sisters Program (BBBS) does provide insight into these impacts (Tierney \& Grossman 2000). The BBBS Program uses a very specific mentoring model that incorporates most of the best practices discussed above, including mentor and youth screening, mentor training, matching requirements, and supervision. The BBBS evaluation analyzed the program for its ability to reduce antisocial activities; improve academic attitudes, behavior, and performance; and improve family relationships after 18 months of the intervention.

The evaluation results reveal strong impacts of the program on specific antecedents of early sexual initiation, including school attachment and family relationships. For example, overall, youth attending the program skipped half as many days of school and a third fewer classes compared to children in the control group. In addition, youth in the program felt more confident 
of their ability to complete their schoolwork than youth not in the program. ${ }^{\mathbf{8}}$ For all of these measures, the impact was stronger for girls than for boys, and for the measure of academic confidence, the impact was strongest among minority females. However, the program did not affect the amount of time youth spent reading, doing homework, or the number of times the youth used a library.

The evaluation also provides evidence that family relationships can be improved through mentoring. The authors theorized that the bonds and trust formed in a successful relationship with a mentor can carry over into other relationships by helping youth to trust, express anger more productively, and become better able to relate to others. Program participants were found to have a better relationship with their parents, which was driven primarily by an increase in the trust that program participants felt toward their parents. ${ }^{9}$ Program participants were less likely than youth not in the program to lie to their parents.

The evaluation revealed few effects of the program on measures of self-concept and peer relationships. Overall, there was no impact on measures of self-worth, social acceptance, or selfconfidence, while only one measure of peer support (emotional support from peers) was significant.

\section{CONCLUSION}

The literature reviewed here provides evidence that youth development programs employ a number of different YD approaches that AFL programs might adopt. These YD programs can have a direct effect on sexual risk-taking behavior, and bolster a number of the protective factors that help youth delay first sex.

Seven relatively well-documented programs designed to reduce or prevent sexual risk-taking behavior have a number of common elements to both their programs and evaluations, suggesting that these elements may enable the programs to measurably impact adolescents' premarital sexual activity and other sexual risk-taking behavior. These elements include the following: (1) one or more social domains are adequately addressed through various interventions; (2) a significant number of participants are included in both the treatment and control or comparison groups; (3) YD activities are strategically implemented; (4) pre- and posttesting of participants is conducted to measure the short-term effects on participants; (5) longitudinal or follow-up testing is conducted to assess longer-term effects; and (6) positive results are seen in desired areas.

There is also evidence to suggest that YD programs can improve parent-child bonding and school success, both of which are protective factors that delay first sex. Some evidence also exists that YD programs can improve emotional well-being, particularly among those groups of youth that are not part of mainstream youth culture. Programs can also work to improve peer relations, but it is not clear whether this type of improvement is consistent with the type of peer relations that delay first sex.

\footnotetext{
${ }^{8}$ Harter's scale of perceived scholastic competence (1985) was used to determine a student's expectation for school success.

${ }^{9}$ The dimensions of the overall parental relationship construct included trust, communication, and anger and alienation.
} 
The two programs in the review by Catalano and colleagues $(2002,2004)$ that reduced parentchild conflict were those that devoted the most resources to working with parents both separately and with their children. These programs combined a strong parental training component with joint parent-child activities to change the parent-child relationship. Parental involvement in administering the program appeared to have less influence on the strength of the parent-child relationship compared to the first two strategies.

Findings from the review also highlight the importance of the distinction between parent-child connectedness and parent-child communication. From the review of AFL grantee program documents, it appeared that grantees worked most often to improve parent-child communication about sex and focused less often on trying to improve parent-child relationships more generally. Moreover, many grantees appeared to struggle with the parental components of their programs. Given the literature emphasizing the importance of strong parent-child relationships in delaying first sex, and programs employing parent training and parent/child bonding activities that can improve the connection between parents and children, OAPP should explore ways to assist AFL grantees to strengthen this aspect of their programs.

Programs in the Catalano and colleagues $(2002,2004)$ study that succeeded in improving attachment to school were similar in that they engaged teachers as well as program participants and parents. These programs provided professional development and training to teachers in addition to youth and family supports. In addition, programs that were effective in enhancing participants' emotional well-being appeared to be most able to provide "support for efficacy and mattering" as the NRC/IOM (2002) report suggests. This was done through such practices as recognizing participant accomplishments and by providing program components such as bilingual education or cultural efficacy exercises to allow distinct populations such as Native Americans and foreign-born youth to feel more a part of mainstream culture. Finally, programs that accomplished improved peer relations did so through teacher training and classroom exercises. It is unclear, however, whether these improved peer relations delay first sex, because we are not certain if the peer cohesion occurred among "low-risk" peers.

As a final point, the review also highlighted how important it is to implement YD strategies properly. This point was made clearly in NRC/IOM's report's discussion of the program features that promote $\mathrm{YD}$ and in the findings from the meta-analysis of mentoring programs. As this literature points out, it is not enough just for programs to engage children in a YD activity with an adult. In order to be effective, that adult-child relationship must be warm, supportive, and caring. Likewise, as Dubois and colleagues (2002) and NRC/IOM (2002) found, mentoring programs in general may have only a minimal effect, but well-implemented ones can be can be quite effective. As such, OAPP should encourage better implementation, using the implementation elements described in this review as a guide. 


\section{CHAPTER 3 REVIEW OF YOUTH DEVELOPMENT COMPONENTS OF OAPP- FUNDED ABSTINENCE PROJECTS}

\section{INTRODUCTION}

The Title XX Adolescent Family Life (AFL) program supports grantees that provide services to promote abstinence from sexual activity and other risk behaviors. The Office of Adolescent Pregnancy Programs (OAPP) in the Office of Population Affairs (OPA) encourages AFL grantees to offer what are commonly called "youth development approaches," which it expects will provide a more holistic approach to preventing adolescent premarital sexual activity. Unlike the more traditional prevention/intervention programs that target specific negative behaviors, youth development approaches such as building self-esteem, promoting bonding with parents and schools, and encouraging future goals and aspirations work to increase developmental assets across a number of different developmental domains (physical, psychological, intellectual, and social). A growing body of literature suggests that strengthening these developmental assets increases the ability of youth to resist many of the ever-present risks that they confront in adolescence (see, for example, NRC/IOM 2002). While they are not empirically tested, it is hoped that youth development approaches, working in conjunction with abstinence education, can be successful in reducing the number of youth engaging in premarital sexual activity.

Currently, OAPP does not have adequate information on the extent to which AFL grantees employ youth development approaches, the types of approaches they employ, and the intensity of these approaches. More information is also needed to understand whether and how AFL grantees are evaluating the youth development approaches they use to determine whether they enhance the effectiveness of the abstinence curricula being used, and whether certain approaches are more or less effective than others when coupled with an abstinence curriculum.

This report summarizes findings on these issues from a review of AFL grantee program documents. We first describe the method we used to review the program documents. Next we discuss findings - to the extent that they could be ascertained from the documents - concerning the characteristics and intensity of the youth development approaches used by the grantees, their evaluation methods, and the challenges they have faced while implementing their programs. The report concludes with recommendations ranging from how the year-end documents' organization can be restructured to provide more complete, consistent, and useful information to how the design of program evaluations can be improved.

\section{METHODOLOGY}

To understand the characteristics and intensity of the youth development approaches used in AFL demonstration projects and the ways in which the grantees attempt to evaluate these approaches, we reviewed the program documents of 45 AFL grantees. For most grantees, these documents included both the year-end and evaluation reports. Because this review was conducted toward the end of 2004, before many of the most recent grantee reports were submitted, we looked mostly at documents reporting about the 2002-2003 program year. 
We developed a data collection protocol to ensure that the three reviewers analyzing program documents did so consistently (see appendix B). This protocol focused on extracting information in five areas:

- organizational characteristics (organizational type, funding status, and personnel information);

- demographics of the target population (the program's catchment area, as well the age, sex, and racial/ethnic characteristics of the children being served);

- program characteristics (the abstinence curricula being used, the positive youth development (PYD) goals addressed by the program, and the characteristics and intensity of the youth development interventions used to address these goals);

- challenges encountered by the programs; and,

- evaluation methods (the evaluation design and instruments).

Below, we discuss in detail how we define youth development approaches and the intensity of these approaches.

\section{DEFINING POSITIVE YOUTH DEVELOPMENT OBJECTIVES}

A major issue with documenting the positive youth development objectives (PYDOs) used by the AFL grantees involves the lack of a precise definition of what a PYDO is. To define PYDOs we began with the work of Catalano and colleagues (2002, 2004), which identifies a set of objectives that PYD programs wish to achieve. These are:

- promoting bonds with prosocial institutions and community;

- promoting bonds with parents/family and parent-child communication;

- fostering resilience;

- promoting social, emotional, cognitive, behavioral, and moral competence (5 PYDOs);

- fostering self-determination;

- ostering spirituality;

- promoting self-efficacy;

- promoting a clear and positive identity;

- fostering a belief in a future;

- recognizing and reinforcing positive behavior;

- providing opportunities for pro-social involvement; and

- enhancing prosocial norms.

In examining the program documents to discern the characteristics of the objectives adopted by AFL grantees, we looked for any program activities that attempted to meet these objectives. Under this framework any number of activities can be classified as a "PYDO" as long as the activity is being offered in a conscious effort to promote one of the above objectives. Examining the AFL grantees in this way ensures that we do not overlook enrichment activities consistent with a youth development objective, but which are not necessarily included in the common list of youth development activities. Programs are coded as pursuing a specific PYDO if program 
documents overtly name the PYDO as a program objective or if the program engages in an activity that the reviewer feels achieves one or more of the PYDOs (see appendix $\mathrm{C}$ for detailed coding conventions).

\section{UNDERSTANDING THE DATA AND FINDINGS}

The reader should keep in mind a number of points when considering the data and findings presented here, and summarized for each program reviewed in appendix D. First, the year-end and evaluation reports varied dramatically in their breadth, depth, and overall comprehensiveness. Some grantees offered quite detailed reports of their activities. But in many cases the documents lacked the detail necessary to understand the characteristics and intensity of the grantee's PYDOs. In some cases, therefore, we may underestimate the extent to which AFL grantees have adopted PYDOs because their reports simply did not discuss these objectives.

Second, the program documents reviewed describe the AFL grantees in their 2002-2003 program year. Accordingly, these documents may not reflect the programs' most recent practices. Many program documents describe plans for the 2003-2004 program year to enhance their youth development strategies and improve their evaluation methodologies. Because these documents were not available we cannot tell whether these enhancements occurred, or what form they took. If they did in fact occur, we may again be underestimating the extent to which these programs have adopted PYDOs as well as the quality of their evaluations.

Third, in most cases the quality of the program documents meant that reviewers could not discern how fully the programs have integrated PYDO with their abstinence curricula. Such integration would mean that program participants received abstinence messages along with and connected to PYDOs, or whether the AFL programs simply have both without relating one to the other for their youth participants. This integration was a major reason for and focus of site visits.

Finally, it is important to note that we could not determine a key aspect of intensity from the program documents, namely how thoroughly engaged program participants are in each activity. While participants may be exposed to a specific abstinence message or PYDO for long periods of time, the impact of these activities will not be as great if the participant is not truly engaged. Accordingly, a key component of our site visit methodology will be observations of the participants attending the program to get a sense of their level of engagement in the program activities.

\section{FINDINGS}

\section{Characteristics of the Grantees in Our Sample}

A brief overview of grantee characteristics provides the context for understanding the bulk of our document review findings. Table 3.1 presents these characteristics. During the 2002-2003 program year, most grantees in our sample were in the second year of their funding cycle (31 programs). Only two programs were in their first year of funding, five in their fourth year, and four in their fifth year. 


\begin{tabular}{|c|c|c|}
\hline \multicolumn{3}{|c|}{$\begin{array}{l}\text { Table 3.1. Program Characteristics of AFL Grantees } \\
(N=45 \text { programs operating at least one year })\end{array}$} \\
\hline CHARACTERISTIC & $\begin{array}{c}\text { NUMBER } \\
\text { OF } \\
\text { PROGRAMS }\end{array}$ & $\begin{array}{l}\text { PerCentage } \\
\text { OF Programs }\end{array}$ \\
\hline $\begin{array}{l}\text { Grantee year } \\
\text { First } \\
\text { Second } \\
\text { Third } \\
\text { Fourth } \\
\text { Fifth }\end{array}$ & $\begin{array}{c}2 \\
31 \\
2 \\
0 \\
5\end{array}$ & $\begin{array}{r}4.4 \\
68.9 \\
0 \\
11.1\end{array}$ \\
\hline $\begin{array}{l}\text { Program setting } \\
\text { School-based } \\
\text { After-school } \\
\text { After-school and summer } \\
\text { NA-parent-focused }\end{array}$ & $\begin{array}{c}2 \\
20 \\
12 \\
1\end{array}$ & $\begin{array}{r}4.4 \\
44.4 \\
27.3 \\
2.2\end{array}$ \\
\hline $\begin{array}{l}\text { Racelethnicity of clients served } \\
\text { Mostly/exclusively white } \\
\text { Mostly/exclusively black } \\
\text { Mostly/exclusively Latino } \\
\text { Mostly/exclusively Native American } \\
\text { Mixed } \\
\text { Other/Missing }\end{array}$ & $\begin{array}{c}3 \\
11 \\
5 \\
3 \\
18 \\
5\end{array}$ & $\begin{array}{r}6.7 \\
24.4 \\
11.1 \\
6.7 \\
40.0 \\
11.1 \\
\end{array}$ \\
\hline $\begin{array}{l}\text { Sex of Clients Served } \\
\text { Both boys and girls } \\
\text { Boys only } \\
\text { Girls only }\end{array}$ & $\begin{array}{c}41 \\
1 \\
3 \\
\end{array}$ & $\begin{array}{r}91.0 \\
2.2 \\
6.6\end{array}$ \\
\hline
\end{tabular}

Most program activities took place in the after-school hours (20 programs). Only 3 programs were designed to take place during the school day, while 12 programs had both after-school and summer program components, and 1 program served parents rather than children.

Nearly all of the programs in our sample served both boys and girls (41 programs), with only one focused exclusively on boys and three focused only on girls. The grantees also served different racial and ethnic groups. Eleven programs either predominately or exclusively served AfricanAmerican children, while five predominately or exclusively served Latino children, three served mostly white children, and three served Native American children. Eighteen programs served a relatively even combination of two or more of these groups. Two programs served immigrant populations and three programs did not give this information. 
To determine how AFL grantees incorporated PYDOs into their activities, we examined the year-end and evaluation documents to learn what kinds of activities they offered and which PYDOs they expected to affect with these activities. As explained earlier, our understanding of how many PYDOs are incorporated in a single AFL program or what approaches are used to achieve PYDOs is limited by the information contained in grantee documents. We searched program documents for any mention of or reference to any of the PYDOs noted above, which for coding purposes we differentiated into 16 categories, coding community and parent-child bonding in separate categories and doing the same for five different competencies (social, emotional, cognitive, behavioral, and moral)

After identifying the presence of a PYDO, we identified the curricula or enrichment activities used by the AFL grantee to influence and/or achieve the objective. This section describes which PYDOs we observed in the program documents, which were most and least common, and examples of the enrichment activities used by AFL grantees to achieve PYDOs.

\section{Observed Positive Youth Development Objectives}

While none of the 45 AFL grantees have attempted to achieve all of the PYDOs, three programs support youth development interventions that target 15 of the 16 objectives. Of these three AFL grantees, Morehouse School of Medicine and the Boston Medical Center do not have programs that attempt to foster spirituality, but their programs intend to achieve all the other youth development objectives. The youth development intervention of Northwest Family Services does not provide recognition for positive behavior but does have components that satisfy the other objectives, including fostering spirituality.

Twelve programs examined in this study have youth development interventions that attempt to meet 13 or 14 of the PYDOs. Of these 12 programs, the most commonly excluded youth development objective is "to foster spirituality." Eleven programs do not explicitly attempt to influence a participant's spirituality. Nine AFL grantees do not mention providing recognition for positive behavior and eight programs do not include components targeted at promoting moral competence.

On the other end of the spectrum, several programs incorporated very few PYDOs. Our inability to identify PYDOs for these programs may not be a result of the quality of the interventions; instead, the AFL grantee may have designed an intervention that captures the ideas of one or several PYDOs but the program documents may not convey this effort. It may also be that programs choose to focus on one or two PYDOs instead of attempting to achieve many objectives through one program. Regrettably, the program documents did not capture this information so it is impossible for us to explain why some programs target very few PYDOs while others attempt to achieve many.

Jackson State University is the only program that does not mention any of the PYDOs in its endof-year report. Program documents indicate differences between this program and most other AFL programs. Jackson State University's program trains school personnel in counseling and 
decisionmaking, and students are offered an abstinence education curriculum. This difference in program design may explain the lack of PYDOs mentioned in reports.

The five most commonly adopted PYDOs are promoting bonding, parent-child communication, and cognitive competence; and fostering resilience and self-efficacy. Of these five PYDOs, two are close to universal. Thirty-six of the 45 AFL grantees set "promote bonding" and "promote child-parent communication/bonding" as main objectives of their youth development interventions, while 31 grantees state that promoting cognitive competence is a central objective of their youth development interventions. Thirty AFL grantees include resiliency and selfefficacy objectives in their youth development interventions.

\section{Approaches to Positive Youth Development Objectives}

AFL grantees implemented a wide variety of youth development approaches, which varied from curriculum-based programs to enrichment and/or recreational activities. While some grantees implemented curricula as the primary means to influence youth development objectives, other grantees used enrichment activities to create a positive youth development approach. As explained earlier, many different activities can be classified as promoting PYD as long as the activity attempts to reach one or more of the PYDOs described by Catalano and colleagues (2002, 2004).

We highlight some of the enrichment activities used to achieve specific PYDOs. While some of the activities were specifically targeted at one objective, many activities were used to satisfy several objectives at once.

As described earlier, we focused on two measures of intensity-duration and frequency. Catalano and colleagues $(2002,2004)$ explain that 80 percent of the "effective, well-evaluated programs" in the study had interventions that lasted over nine months, but that less than 45 percent of the programs in the excluded (i.e., ineffective) group had interventions that lasted nine months or longer. It has been argued that the more effective youth development programs have long-term interventions with frequent meetings. The thought is that the more intense the intervention a youth receives, the better the chance that PYDOs will be achieved.

\section{MEASURING INTENSITY}

We use two measures, duration and frequency, to describe the intensity of the youth development interventions, defined as follows:

1. Duration: How long the intervention lasts in calendar time. Interventions lasting at least one calendar year (12 months or more) were classified as "high duration." "Medium duration" was defined as lasting between one school year and one full year. Programs lasting less than one school year were classified as "short duration."

2. Frequency: How often participants could be exposed to the intervention in a given period (e.g., 2 hours per week). "High frequency" was defined as exposure to the intervention for more than five hours per week, on average, over the duration of the intervention. Programs 
offering two to four hours a week of exposure were considered "medium frequency." Programs offering less than two hours of exposure a week, on average, were considered "low frequency."

Thirty grantees supplied enough information in either their year-end reports or during site visits for us to classify them into one of the nine categories. We used these two variables to create a third measure- "intensity." A program is identified as low intensity if it lasts less than a full school year and offers less than two hours per week of curriculum or enrichment activities. Medium intensity interventions provide two or more hours per week of curriculum or enrichment activities for up to a full year. Finally, an intervention that offers curricula or enrichment activities two hours or more per week for more than a full year or offers five or more hours per week for at least 10 months is classified as high intensity. Table 3.2 shows the nine categories of intensity and indicates the number of programs in each (two categories had no programs). Table 3.3 classifies each AFL program according to its intensity.

\begin{tabular}{|c|c|c|c|}
\hline \multicolumn{4}{|c|}{$\begin{array}{l}\text { TABLE 3.2: FREQUENCY AND DURATION OF AFL PROGRAMS, AND } \\
\text { INTENSITY }\end{array}$} \\
\hline \multirow[b]{2}{*}{ Duration } & \multicolumn{3}{|c|}{ Frequency } \\
\hline & $<2$ hours per week & 2-4 hours per week & $5+$ hours per week \\
\hline $\begin{array}{l}\text { Less than a school } \\
\text { year }\end{array}$ & $\begin{array}{c}\text { LOW } \\
\text { (10 programs) }\end{array}$ & $\begin{array}{c}\text { MEDIUM } \\
(2 \text { programs })\end{array}$ & $\begin{array}{c}\text { MEDIUM } \\
(1 \text { program })\end{array}$ \\
\hline $\begin{array}{l}\text { Between a school } \\
\text { year and a full year }\end{array}$ & $\begin{array}{c}\text { LOW } \\
(2 \text { programs })\end{array}$ & $\begin{array}{c}\text { MEDIUM } \\
(10 \text { programs })\end{array}$ & $\begin{array}{c}\text { HIGH } \\
(0 \text { programs })\end{array}$ \\
\hline $\begin{array}{l}\text { More than a full } \\
\text { year }\end{array}$ & $\begin{array}{c}\text { LOW } \\
(0 \text { programs })\end{array}$ & $\begin{array}{c}\text { HIGH } \\
\text { (2 programs) }\end{array}$ & $\begin{array}{c}\text { HIGH } \\
\text { (3 programs) }\end{array}$ \\
\hline
\end{tabular}

The majority of programs fall into the low or medium intensity categories, with only five programs being considered high intensity. The Be'er Hagolah Institutes, Baptist Children's Home Ministries, The Children's Council, Fifth Ward Enrichment Program, and Healthy Mothers, Healthy Babies programs provide long-term interventions of at least 10 calendar months (mostly these programs are multiyear), during which youth receive at least two and usually more hours per week of curricula and enrichment activities.

It is not clear whether AFL grantees have a hard time providing long-term youth development interventions, or whether they are not trying to do so. One of the challenges programs face is recruiting and retaining participants over time. It may be difficult for these grantees to keep participants engaged in programs that require a commitment of many months. To remedy this, some programs shorten the duration of their intervention but increase the frequency of meetings and/or enrichment activities to have more contact with the participants during a shorter period of time. This solution is apparent in the high concentration of programs that offer between two and 


\begin{tabular}{|c|c|c|c|}
\hline \multicolumn{4}{|c|}{ TABLE 3.3: AFL PROGRAMS REVIEWED/VISITED, CLASSIFIED BY INTENSITY } \\
\hline \multirow{2}{*}{ Duration } & \multicolumn{3}{|c|}{ Frequency } \\
\hline & $<2$ hours per week & 2-4 hours per week & $5+$ hours per week \\
\hline $\begin{array}{l}\text { Less than } \\
\text { a school } \\
\text { year }\end{array}$ & $\begin{array}{ll}> & \begin{array}{l}\text { Alabama State University } \\
(\text { AL) }\end{array} \\
> & \text { BETA Center(FL) } \\
> & \text { Boat People SOS (VA) } \\
> & \text { Jackson State University } \\
& \text { (MS) } \\
> & \text { JOVEN (TX) } \\
> & \text { Mercy Hospital of Pittsburgh } \\
& \text { (PA) } \\
> & \text { St. Luke's Roosevelt Hospital } \\
& \text { (NY) } \\
> & \text { To Our Children's Future } \\
\text { with Health (PA) } \\
> & \text { University of Alabama (AL) } \\
> & \text { YMCA of Cumberland (MD) }\end{array}$ & $\begin{array}{l}>\quad \text { Colorado State University } \\
(\mathrm{CO}) \\
>\quad \text { Wise Women Gathering } \\
\\
\text { Place (WI) }\end{array}$ & $>\quad$ Clippers (NC) \\
\hline $\begin{array}{l}\text { Between a } \\
\text { school } \\
\text { year and a } \\
\text { full year }\end{array}$ & $\begin{array}{l}\text { Catholic Social Services of } \\
\text { Miami Valley }(\mathrm{OH}) \\
>\quad \text { Dallas Independent School } \\
\text { District } 2(\mathrm{TX})\end{array}$ & 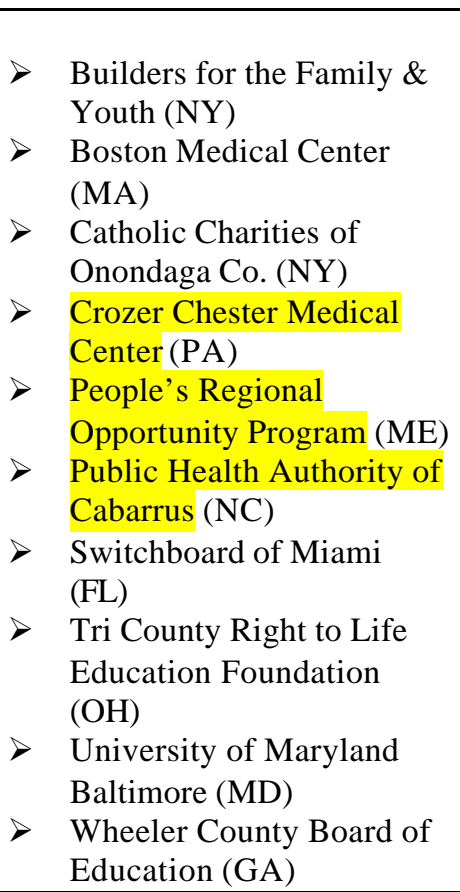 & \\
\hline $\begin{array}{l}\text { More than } \\
\text { a full year }\end{array}$ & ------ & $\begin{array}{l}>\quad \text { Baptist Children's Home } \\
\text { Ministries (TX) } \\
>\quad \text { The Children's Council } \\
(\mathrm{SC})\end{array}$ & $\begin{array}{ll} & \text { Be'er Hagolah Institutes } \\
& (\mathrm{NY}) \\
> & \text { Fifth Ward Enrichment } \\
& \text { Program (TX) } \\
> & \text { Healthy Mothers, } \\
& \text { Healthy Babies (NC) }\end{array}$ \\
\hline
\end{tabular}


four hours of activities per week for between a school year and a full year. The high number of programs that fall into the low intensity category illustrates that programs may find it easier to provide short-term interventions for short periods of time per week. However, the Baptist Children's Home Ministries program, which is a long-term, intense program, has not listed recruitment and retention as a major challenge. In fact, this program listed too much interest in the program and too many participants as a major challenge they are facing. This belies the idea that intense programs might have more trouble recruiting and retaining participants, and suggests that some programs may not yet have figured out how to deliver their messages in ways that are appealing to youth.

\section{Challenges facing AfL Programs}

Operating a risk behavior prevention program of any scope for adolescents is a difficult task. These types of programs face complex issues associated with factors that extend far beyond teenagers engaging in risky behavior at an early age. The issues related to the risky behaviors include broader social, cultural, and economic factors. These obstacles pose even greater challenges to those who design and operate prevention programs.

- Many of the abstinence education programs currently receiving OAPP funding have faced challenges in implementing their interventions and/or reaching their desired outcomes in the anticipated time period. Of the 45 program year-end reports that we thoroughly examined, 39 programs reported facing at least one challenge and 22 reported encountering more than one challenge. Table 3.4 shows the common challenges encountered by these programs.

Table 3.4: Challenges Facing AFL Programs

\begin{tabular}{|l|l|l|}
\hline \multirow{2}{*}{ CHALLENGE } & \multicolumn{1}{|l|}{$\begin{array}{l}\text { AFL PROGRAMS } \\
\text { REPORTING ANY } \\
\text { CHALLENGE } \\
(\boldsymbol{N}=39)\end{array}$} \\
\cline { 2 - 3 } & NUMBER & PERCENT \\
\hline Recruiting and retaining youth participants & 12 & 31 \\
\hline Little or no involvement of parents or other family members & 9 & 23 \\
\hline $\begin{array}{l}\text { Staffing (e.g., high job turnover rate, inadequate number of staff, } \\
\text { training) }\end{array}$ & 12 & 31 \\
\hline $\begin{array}{l}\text { Difficulty in forming or stabilizing relationships with schools or } \\
\text { organizations }\end{array}$ & 8 & 21 \\
\hline Evaluation process (e.g., design, data collection, survey instruments) & 13 & 33 \\
\hline Other challenges reported (e.g., funding, program intensity) & 7 & 18 \\
\hline
\end{tabular}


For the programs that listed more than one challenge, the common pair of challenges is the difficulty in recruiting youth and parent or family involvement. Four AFL programs reported experiencing difficulties in both retaining or recruiting youth participants and involving parents or other family members in the program. Characteristics of these programs that may have some impact on participation levels include a predominantly culturally-sensitive target population (e.g., Latinos) and activities offered in a combination of time periods (e.g., in school, after school, or during the summer). It is likely that those coming from different cultural backgrounds (and even more so, those with language barriers) may feel less inclined to participate in any activity outside of their day-to-day life activities. This may be particularly true for families who live in economically disadvantaged conditions. In addition, families from lower income backgrounds are more likely to hold jobs with nontraditional working hours or even hold more than one job, making them less able to participate in program activities.

Challenges Associated with Youth and Family Participation. Many of the 39 AFL programs that reported facing any challenges stated difficulties recruiting or retaining program participants (12) or getting parents or other family members involved in the program (9).

Some of the issues underlying the low level of participation (either recruitment or retention) among youth as reported by the programs involve lack of interest or the inability to participate due to program intensity or other personal commitments. AFL programs that rank medium in program intensity are more likely to have trouble recruiting or retaining youth participants. Activities that are held over a longer period of time and require frequent attendance may impact a youth's ability or desire to participate. Consequently, this will negatively affect the intensity of the interventions (i.e., duration and frequency of activities may need to be shortened) or the evaluation process (i.e., inadequate number of participants for inclusion in the control or comparison group).

Another factor that can affect participation level stems from the PYDOs around which program activities are developed. Of the programs that reported youth participation as a challenge, 7 embedded more than 10 PYDOs in their interventions. Activities that involve a large number of objectives may impact youth participation. One suggestion is that participants who feel an activity is attempting to achieve too much may get discouraged or disinterested. For example, a typical community service learning project may subjectively include several PYDOs, such as (1) promoting bonding; (2) promoting social competencies; (3) fostering self-determination; (4) fostering self-efficacy; (5) fostering a clear and positive identity; (6) fostering beliefs in the future; (7) providing opportunities for prosocial involvement; and (8) fostering prosocial norms. In other words, the community service learning project may not only want young people to help in their community, but the project may also want the participants to exhibit leadership by taking on various positions in their community in order to achieve some of the objectives mentioned.

The low level of parent or family involvement is reportedly due to cultural or language barriers, or inflexible hours of operation. Solutions offered by some of these programs include increasing contact with parents to raise awareness or comfort in participating, or becoming more flexible by operating during the weekends. Nonetheless, many of the programs facing low parent or family participation ensure that parent-child bonding is an element included in their activities. For example, family involvement activities may include evening workshops for parents to discuss 
techniques for communicating with their child, review the abstinence education curriculum, and receive updates on their child's progress in the program.

Problems with Project Staffing. Twelve AFL programs noted difficulties with project staff, including high job turnover rate, inadequate number of staff, and training. There was turnover in the schools where many of the programs housed their activities. Consequently, programs had to rehire and retrain new staff, often delaying the project.

Training for project staff (e.g., peer educators, teachers, and counselors) was reported to be a major challenge. A few programs mentioned a need for bilingual staff and the resources (e.g., funds, bilingual instructors) to train in a different language. Other programs simply could not get all staff to be on the same page during the training sessions-another cause for project delays. The level of training for project staff is unclear based on the year-end reports.

Missing information on the intensity of these programs' interventions makes it difficult to determine if there is a relationship between staffing difficulties and the intensity of the activities.

Obstacles in Establishing School and Community Partnerships. Difficulties in establishing partnerships with area schools and community-based organizations were experienced by eight AFL programs. Programs reported that problems associated primarily with project staffing prevented potential partner organizations to be on board. For instance, schools in one district had such high turnover that it became difficult for school personnel to agree to the program.

Another example is the program that experienced severe hiring difficulties and therefore, the activities could not get started until after the school year had begun. Consequently, the program was met with some resistance, and the intensity of the activities had to be reduced to accommodate other ongoing school activities.

Challenges in Conducting Their Evaluation. Of the 39 AFL programs that reported facing any challenge, the largest number (13) encountered difficulties with the evaluation process. Problems include the evaluation staff, experimental design, data collection, and the management information systems. Of these 13 programs, 7 intended to have a quasiexperimental design in which a matched comparison group was involved, and 3 programs conducted follow-up beyond the immediate posttest. However, in some cases the difficulty of recruiting participants hindered the ability to form a comparison group. These programs had trouble getting youth to join the program, let alone having an adequate number of youth participate in a comparison group for the program. Also, retention of youth participants was such a challenge that follow-up became an even bigger obstacle.

Seven programs designed their survey instruments to allow for measurement of knowledge, attitudes, and behavioral intentions, and two programs measured only knowledge and attitudes. Given the relatively short duration of these programs, an even bigger challenge is accurately measuring actual behavior, which is often measured through follow-up to get a sense of a program's longer-term impact. Four programs reported being able to design an evaluation that 
measured actual behavior, yet only two of the se programs conducted a follow-up beyond the immediate posttest.

\section{IMPLICATIONS OF THE DOCUMENT REVIEW FOR AFL PROGRAM EVALUABILITY}

Overall, evaluation is the most frequently reported challenge faced by the AFL abstinence education programs. Grantees reported difficulties with evaluation staff, the process of collecting and analyzing the data, inadequate tools of measurement, and/or the experimental design. Because each program differs in its evaluation, it is a challenge to conduct an evaluability assessment of the youth development components in these programs and their contributions to program outcomes. To overcome this challenge, one strong recommendation to OAPP is to mandate that future demonstration projects have an evaluation plan based on rigorous scientifically based research methods. To accomplish this OAPP may have to adjust the monetary allocation for the evaluation of program services. With respect to this recommendation, programs should consider:

- an evaluation design that consists of random assignment to an experimental and a control group;

- a quasi-experimental design with matched comparison groups if a random assignment experiment is not possible; or

- a design that allows for multiple baseline or longitudinal results (i.e., follow-up) if adequate numbers of participants are not available to support random assignment or comparison group designs, and even if such groups are available.

\section{Understanding How the AFL Grantees ARE Evaluated}

In this section we discuss findings from a review of the evaluation documents available from the AFL grantees in our sample. We briefly discuss the major components of a strong evaluation design to provide a benchmark by which to assess the AFL evaluation documents. Then, we discuss the strengths and weaknesses of the AFL grantee evaluations across each of these components. It is important to note at the outset that, given the limited funds that AFL grantees have to evaluate their programs, we did not expect them to excel in each component. Instead, this analysis is designed to assess where the grantees are in the evolution of their evaluation strategies, and to provide recommendations concerning how these evaluations could be strengthened within the context of limited resources.

\section{Dimensions of a Strong Evaluation Design}

A number of different characteristics define a strong evaluation design. First, evaluations must be clear about the theoretical linkages between a program's intervention components and specific outcomes. Simply, if no clear theoretical reason exists to believe that a certain program activity will change an outcome, the impact of the activity on that outcome should not be assessed. Conversely, all outcomes that a program expects its activities to affect should be assessed. Understandably, this latter issue may be particularly relevant for the AFL grantees, because 
many grantees may not be as concerned about examining outcomes related to their PYD activities as they are with outcomes related to sexual risk-taking behavior.

Second, evaluations should attempt to employ strong evaluation designs, and the interpretation of the evaluation results should be in line with the limits of the design. For example, programs that employ a process study to evaluate a program should limit conclusions to those that deal with the administration of the program components, and should not attempt to make causal statements about outcomes. Finally, evaluations should employ instruments that accurately measure both the level of participation in the program and the desired outcomes.

Given these characteristics of strong evaluation designs, four different aspects of AFL grantee evaluations were reviewed:

- the evaluation's understanding of intervention components and how these may impact specific outcomes (logic model);

- the evaluation's design and methods;

- the evaluation's ability to measure the extent to which the participants are exposed to the intervention components (dosage); and

- the evaluation's ability to measure and collect data on specific youth outcomes.

The findings from our review of these aspects of the AFL grantee evaluation documents are discussed below.

Do the evaluations demonstrate a strong understanding of the intervention components and how they may affect outcomes?

For the most part, the evaluation reports appear to have a solid grasp of the intervention components. Most contained at least a modest description of the program design and discussed the major activities that take place within the program, although in some cases these descriptions appeared to be copied verbatim from the year-end reports. In addition, several program evaluations reported findings from process studies, which allowed the evaluator a more in-depth look at the implementation of program components.

Most of the program evaluations, however, did not contain an explicit logic model that outlined the theoretical link between the activities offered in the program and outcomes that are measured in the outcome evaluation. Evaluation documents most often contained a "measurable objectives" section, which discussed the objectives of the grantees and how the evaluation measures them, but did not attempt to provide a theoretical justification for the link between a specific intervention component and a specific outcome. We believe that this is an important piece of evaluation methodology; in some cases where it was missing, it was not clear how a program intervention could achieve the specified objective. For example, while program documents might state that a program component such having a parent watch a theatrical performance will lead to better parent-child communication, it is unclear why this would be the case. 
In some cases, however, the link between program activity and measured outcomes is drawn quite clearly. One notable example comes from the evaluation of the Catholic Charities of Onondaga County evaluation. The evaluation discusses the peer mentoring component of the program and how this component will help build specific developmental assets. The evaluation cites research by the Search Institute on how opportunities to develop useful roles and to be of service to others are important empowerment assets. The evaluation of the DARE To Be You program conducted by Colorado State University also does an exceptional job of relating program components to specific outcomes and is one of the few evaluations to contain a logic model.

\section{How do the evaluations vary in their design?}

The AFL grantees used a variety of designs to evaluate their programs. The most common evaluation design was a quasi-experimental design with a comparison group (17 programs). Ten other programs used a pre-test/post-test design with no comparison group, while 8 other programs employed other, less rigorous evaluation methodologies like process studies or focus groups of program participants. It is also important to note that three evaluations, those of Catholic Charities of Onondaga, Colorado State University Cooperative Extension, and Switchboard of Miami Inc, used a random assignment design to measure program impact. Eight other programs either did not have a evaluation document or their evaluation documents did not specify an evaluation design.

Another important component of the evaluation design is the extent to which program participants are followed over time. Given that one potential issue with the AFL program interventions is that the effects may fade over time, it is important to understand whether the positive effects of the abstinence intervention persist over time and for how long. Eleven program evaluations (for which we had an evaluation report) had some sort of follow-up. The follow-up periods varied across the program from as short as one month after the end of the program to as long as two years. In addition, at least 2 of the 11 programs also conducted followup at multiple points in time. For example, Colorado State University's evaluation planned to follow up with participants at 6, 12, and 18 months after the end of the program in an attempt to determine if the benefits of the program endured.

\section{Do the evaluations measure the extent to which the participants are exposed to the intervention components?}

Interestingly, while most evaluation documents discussed the duration and intensity of the program components, few discussed the actual participation rate of the students in the program. While recruitment and retention of program participants was cited as a challenge for a number of programs, few evaluations focused on understanding the specific dosage that participants across the programs received and how issues with low participation rates may affect outcomes. Currently, it is unclear whether these rates are simply not reported in the evaluation or whether the grantee or evaluator collected them.

Participation rates are an important aspect of program evaluations for a number of reasons. These rates measure program fidelity by giving an indication of the extent to which programs are 
actually serving the children they intend to serve. For example, the evaluation of JOVEN's TEJAS project is one of the few evaluations to report the overall participation rate of program participants. The evaluators find that 87 percent of program participants beginning the program completed the entire intervention cycle, indicating a high level of participation.

Equally important, participation rates helps to qualify outcome findings. If outcome measures are positive despite low participation in the program, this may indicate, all else being equal, that the intervention is more powerful than the results imply. Conversely, it may be the case that interventions that do not show positive results may work, but participants have not had enough exposure to the intervention.

How well did the evaluations measure and collect data on specific child outcomes, particularly those related positive youth development objectives?

In general, program evaluations did a relatively good job measuring the outcomes related to sexual behavior and intention. We found that 14 program evaluations measured sexual behavior, along with sexual intention, attitudes, and knowledge. Eighteen of the programs did not measure behavior, but only sexual intention, attitude, and knowledge, while five evaluations only measured attitudes and knowledge. Information on the remaining five programs was not available.

Many of the evaluations also did a good job measuring PYD outcomes when PYDOs were present in the program. For example, the "Healthy Choices" evaluation measured self-esteem, self-concept, attachment to school, and parent-child communication. The "Teens Taking Charge" project provided perhaps the most innovative measures of difficult concepts such as parent-child connectedness through the use of a "communication tally sheet," which documents the amount of time the child talks to an adult, the subject matter, and person with whom the child speaks. While these two programs represent some of the more comprehensive evaluations in terms of their measures, most programs at least attempted to measure parent-child communication and improved self-concept or self-esteem if these were addressed by the program activities.

\section{Overall assessment of program evaluations}

The evaluations varied quite substantially from grantee to grantee. Few evaluations excelled across all of the dimensions discussed above, but the evaluations of the "Healthy Choices" and the "DARE to Be You" programs stand out as two strong evaluations in terms of design and measurement of outcomes.

To better understand the extent to which PYD approaches improve the effectiveness of abstinence curricula, perhaps the best design would involve randomizing program participants into three groups: (1) students receiving abstinence education only; (2) students receiving abstinence education and engaging in positive youth development interventions; (3) and a control group. While this may be impossible for the AFL grantees to implement, OAPP should move to encourage that the designs of AFL grantee evaluations at least include a comparison group not receiving the grantee's services, whether randomly assigned or not. In addition, one of the most 
important weaknesses across the evaluations overall is their lack of participation data. Grantees and their evaluators should be encouraged to collect this information as well. 


\title{
CHAPTER 4
}

\section{SITE VISITS}

The final study activity involved selecting AFL-funded projects that were judged to have significant levels of YD activities and visiting them to forward our understanding of how abstinence and YD program content were being presented and integrated. An equally important site visit goal was assessing the evaluability of these programs and whether an evaluation would be able to peel apart the independent effects of the abstinence and YD components.

The document review summarized in chapter 3 was a starting place to identify site visit candidates. In addition to what we learned from these reviews, OAPP project officers rated projects under their supervision for the extensiveness of their YD activities and the degree to which abstinence program components benefited from the YD elements. Based on these two approaches we selected seven grantees for site visits. These are:

1. Peer Leader Program (PLP), People's Regional Opportunity Program—Portland, Maine

2. The Real Deal, Be'er Hagolah Institutes-Brooklyn, New York

3. Leadership 101, Crozer-Chester Medical Center-Chester, Pennsylvania

4. Teaching Responsible Actions in Life (TRAIL), Public Health Authority of Cabarrus County-Kannapolis, North Carolina

5. The Children's Council—Lancaster, South Carolina

6. Family Action Model for Empowerment (FAME), BETA Center-Orlando, Florida

7. Fifth Ward Enrichment Program—Houston, Texas

Our first task for the site visits was to understand the basic components of the AFL programs, program history, and current directions. This chapter presents summaries of basic program activities, which have been reviewed for accuracy by program representatives. During site visits we also paid close attention to the individual youth development components, the abstinence education and curricula being implemented, and the combination of the two. We also examined aspects of current evaluation activities and considered options for more extensive evaluations that OAPP might want to undertake. These latter two aspects of our site visit agenda are discussed in the next two chapters of this report.

\section{PEER LEADER PROGRAM, PEOPLE'S REGIONAL OPPORTUNITY PROGRAM- PORTLAND, ME}

\author{
AUSPICES \\ The Peer Leader Program (PLP) is a resiliency-based prevention program located in four \\ Portland, Maine public housing neighborhoods. PLP is one component of the PROP Youth \\ Resiliency Project, which is committed to enhancing the strengths of youth, their families, and \\ the communities they live in. These middle school age youth are primarily from immigrant \\ families from the regions of Somalia, Sudan, Laos, and Vietnam. The PLP gives youth access to
}


the kinds of relationships, experiences, and skills that support positive youth development, healthy decisions, and the ability to be resilient throughout their lives. By letting youth guide the process, plan activities, and evaluate the program and their own progress, PLP creates opportunities for youth to lead.

\section{PROGRAM History}

With funding from the Portland Housing Authority in 1987, PROP staff created PLP in two Housing Authority project neighborhoods as an independent youth project. Nineteen youth, ages 14-16, were identified by residents as natural leaders (not necessarily "positive," just leaders) and selected to participate in the first year of the project. These youth met once a week in a retreat format and contributed to their communities through different activities. The program was built on a peer leader structure where youth engaged and mentored one another.

Several years after its inception, PLP received federal money to continue, expand the program to four neighborhoods, and serve over 100 youth. From 1998-2003, the Office of Population Affairs supported the program with over $\$ 900,000$. Kids Clubs were created and served youth from 7-17 years old. In 2000, the Youth Resiliency Project was created and a major philosophical shift began. In 2002, the program underwent a comprehensive self-assessment and redesigned itself from a deficit/risk-based model to a resiliency-based model. The redesign allowed for youth staff and one manager to whom the youth staff reports. In 2004, the PLP received another grant from the AFL to continue enhancing the design.

As a program of the Youth Resiliency Project, PLP premises its approach on a belief that youth are naturally strong and rich with leadership, honesty, creativity, courage, curiosity, communication, insight, and determination. Staff work to be sure that this belief is reflected in how staff talk with and treat youth, and what expectations they have for youth behaviors.

\section{Program AND SERVice COMPONENTS}

Core Groups: Groups are offered to all children in a variety of capacities. Peer groups are for middle school-age kids, Teen Groups are for high school-age kids, Kids Club is for youths in 1st-3rd grades, Prep Groups are for 4th-6th graders and Special Interest Groups are for all ages depending on the interest. All sites have at least one Teen Group (with 3-12 teens in each group), all sites have at least one Peer Group, and Kids Club, Prep Groups and Special Interest Groups exist based on need and time available at each site. All groups are limited to 12 kids to allow for maximum participation. At the first group meeting, youth are asked to demonstrate why they want to be a part of the group and they are asked why they want to be in PLP. Examples of groups include Boston Celtics Basketball Game Group (several boys wanted to attend a Boston Celtics game and raised money for the transportation and tickets by hosting bake sales), the Hip-Hop Group (hosted a dance and a Halloween Party and raised money for refreshments and a DJ), and the Museum Group (a group of young kids who formed a relationship with the Portland Art Museum, used studio space at the museum to sketch artwork in the museum and create their own artwork, and then displayed their work for the Portland community). Some other projects include a community dance, Artists Without Borders (a fundraiser 
for Doctors Without Borders), young kids helping cater a YMCA function, and kids working on their demo tapes.

Touchstone Function: Each site provides a safe place for adolescents to check in, get help, connect with a site coordinator and other youths, and to feel safe and guarded. Activities include after-school or after-dinner drop-in times each week, street-based activities and outreach to youth at natural gathering places within the neighborhoods, and support for youth to take advantage of other resources PLP is connected to (such as the United Way Summer CHAMPS scholarship program, which pays for and sends innercity youths to summer camps).

$>$ Support for Older Teens : Each neighborhood works to engage and support youth 16-19 as mentors, volunteers, and activists within the program and the community. Often, these teens are linked up with a Kids Club Group or a Peer Group to help the younger kids achieve their group's goal.

Family/Community Outreach and Stakeholder Input: PLP hosts community forums in each neighborhood several times a year to hear from parents and other adults in the community. The forums are an opportunity for stakeholders to tell PLP staff what they think of the program, what they see happening in the neighborhood and how they want PLP to be involved. Also, there is a time each week when staff go out to talk with the parents and family of PLP youth. This time, called Family Outreach, is used for listening to parents' concerns, telling positive stories about the child's participation at PLP, and having parents fill out and sign participant records.

\section{Collaboration}

PLP has two strong collaborative relationships. The first is with United Way and the second is with the City of Portland Department of Public Health (contracted through the Office of Substance Abuse). United Way funds and runs a program called Summer CHAMPS that gives all children in the Portland public school system $\$ 1,000$ for summer camp. PLP helps link kids up with the Summer CHAMPS program and also takes the kids to camp, explains what camp is to refugee families who have a negative idea of "camp," and also translates anything necessary for families who do not speak English. Currently, there are six Somali camps in the state of Maine-an accomplishment that is largely due to Summer CHAMPS's efforts in getting city kids to camp. PROP is a subcontractor of the Office of Substance Abuse to run the Leadership and Resiliency Program (LRP) curriculum, an evidence-based model, with the peer leaders. This program is more culturally competent than the previous curriculum used-that is, it is easier to make its concepts sensible for the Somalis, Sudanese, Cambodians, and Vietnamese youth and families served by PLP because it has greater flexibility and adaptability. 


\section{Program PARTicipants}

\section{Key Characteristics}

of Participants

Percent

\begin{tabular}{|l|l|}
\hline Race / ethnicity & Black (African and African-American): 55 \\
& Asian: 25 \\
& White: 20 \\
& Hispanic: 1 \\
\hline Native English speakers & Mostly not \\
\hline Ages & 12 and under: 59 \\
& $13-14: 33$ \\
& $15-17: 7$ \\
& 18 and above: 2 \\
\hline Low-income (200\% of poverty) & 100 \\
\hline Sexually active & Mostly not \\
\hline
\end{tabular}

\section{STAFF}

Each of the four Peer Leader Program sites is staffed by a full-time site coordinator responsible for planning and facilitating youth activities, and a part-time youth staff between the ages of 1721 who cofacilitates groups. Volunteers of all ages also work at the sites when youth are present. Youth staff are a mix of former program participants and other older youth with a background of working with children. A program manager directs PLP and occasionally visits the sites.

\section{FUNDING}

The majority of PLP's funding comes from the AFL OAPP grant. Other money comes from the Office of Substance Abuse, the City of Portland Community Development, United Way, One Maine One Portland Coalition, and the Portland Housing Authority. The rest of the PLP operating budget is supported by in-kind volunteers and donated space.

\section{THE REAL DEAL (TRD), BE'ER HAGOLAH INSTITUTES-BROOKLYN, NY}

\section{AUSPICES}

Be'er Hagolah Institutes (BHI) is a community-based organization that provides comprehensive educational and social services to immigrant and second-generation immigrant youth from Western Russia and Eastern Asiatic Russia. BHI provides educational, recreational, psychological, socioeconomic, advocacy, ESL, and remedial interventions for this population. The Real Deal (TRD) is a comprehensive abstinence education program for the young females 
enrolled in the Be'er Hagolah Institutes high school. The AFL grant that supports TRD is the first one BHI has received.

\section{PROGRAM History}

BHI School has been in existence for 26 years and serves 1,000 students from kindergarten through 12th grade. The school serves both boys and girls from kindergarten through 12th grade, but they are kept separate during school hours. The Real Deal (TRD) serves 97 girls enrolled in 9th-12th grades (about 60 percent of all girls enrolled in the high school).

\section{Program AND Service CoMPonents}

Girls receive the intervention in 45-minute periods, three periods a day, four days a week, for a total of 540 minutes per week. The girls are split into four groups and participate in the interventions in their groups. The girls receive three of the following core interventions each day in different combinations. The core requirements of the program are:

- Abstinence education: This section follows a curriculum and also has additional components. Sex-in-advertising and media components are incorporated. (Examples: sides of buses, newspapers, radio, TV, etc.) This component addresses self-esteem.

- Communication skills: Communication is taught through writing. The teacher gives short writing assignments that may be personal in nature or may involve interviewing children. The purpose of this is to be inspirational and motivating.

- Health and nutrition: Many topics are covered in these sessions, including drugs, alcohol, nutrition, smoking cigarettes, and so on. The teacher always allows for a 15minute question-and-answer period in which the girls can ask anything they want about health and nutrition. They often talk about eating disorders or issues they see on TV.

- Peer mentoring workshops : A teacher acts as a facilitator until a peer mentor is chosen from the group, who is then responsible for leading the group in discussions. The teacher manages the selection process and the peer mentor gets training from the teacher. Issues raised in conversations are taken from a curriculum designed by BHI. Some peer mentors use role-playing, diagrams, and other devices to help lead the conversations.

- Production activity: Drama, choir, dance, costumes, props, and scenery are used. This promotes self-esteem for students who have difficulties with more standard routes to academic success. (They reference Gardener's multiple intelligences.) This activity, which happens every day, gives them another area of concentration and keeps them busy into the afternoon and into the evenings.

- Sports: Girls play organized sports with their teacher. Girls may opt not to participate and can, instead, run or walk laps around the gym. 
- Electives: Girls choose among art, job readiness, conflict resolution, technology, and home economics. This time is also sometimes used for tutoring for their school exams.

The staff of TRD attempts to include parents in the program in a variety of ways. Unfortunately, the nature of the culture of Russian and East Russian immigrants and recent immigrants does not allow for easy collaboration between the parents and the students. Many parents are ashamed that their daughter participates in the program because of the cultural taboo of sex as a topic and, therefore, do not want to attend parent meetings or workshops. Many parents fear their neighbors or peers will see them attending a TRD event. TRD staff have begun offering parent workshops after PTA meetings so parents can simply stay after, instead of being seen at the school out of context. In addition, TRD staff tries to dispel some of the negative connotations associated with the program. Unfortunately, parent involvement is still relatively low considering the number of girls that participate. This is a major focus of TRD staff. Parental workshops cover topics such as nutrition, sexual education, communication, and updates on the program's progress.

\section{CURriculum}

TRD implements "Sex Can Wait" as well as "Pick and Choose" for their abstinence education components but modify them in many ways. "Pick and Choose" is an abstinence workbook that the program remains mostly loyal to. The art classes follow a fairly strict curriculum to teach drawing, painting and other art skills. Also, the peer-mentoring component follows a curriculum designed by BHI for its children's specific needs.

\section{COLlaboration}

BHI collaborates with three social work agencies (Interboro Agency, Comprehensive, and the Jewish Board of Children and Family Services) on an as-needed basis. In addition, BHI has strong collaborations with the Board of Education, Head Start, and social workers and therapists from various agencies. For the job readiness component of the program, BHI has made strong alliances with several local companies (a local district attorney's office and Queens Law Associates) and health-related organizations.

\section{PROGRAM PARTICIPANTS}

\section{Key Characteristics} of Participants

\section{Percent}

\begin{tabular}{|l|l|}
\hline Race / ethnicity & Russian, East Russian: 100 \\
\hline Native English speakers & Mostly not \\
\hline Ages & $14-18: 100$ \\
\hline Low-income (200\% of poverty) & Not Ascertained \\
\hline Sexually active & Not Ascertained \\
\hline
\end{tabular}




\title{
STAFF
}

The Real Deal's staff is comprised of BHI School teachers and administrators. This includes the Director, Administrative Director, Assistant administrative director, sexual education teacher, program advisor, sports/nutrition/health teacher, two workshop leaders, communication skills teacher, remedial director and an arts teacher.

\section{FUNDING}

Sixty percent of The Real Deal's funding comes from the OAPP grant. The other 40 percent of funding comes from in-kind donations and the school budget. The school is taking on more and more of the operational costs TRD incurs. BHI just received a large grant to begin developing a similar program, curriculum, and interventions for its male youth.

\section{LEADERSHIP 101, CROZER-CHESTER MEDICAL CENTER-CHESTER, PA}

\begin{abstract}
AUSPICES
Leadership 101 (PL 101) operates out of the Wellness Center of the Department of Pediatrics at Crozer-Chester Medical Center, which in turn is one of five hospitals of the nonprofit CrozerKeystone Health System. The Wellness Center is engaged in many aspects of youth development, including several interrelated activities around promoting healthy lifestyles and preventing risky behavior, and strategic planning for youth development for the whole Chester community. The AFL grant that supports PL 101 is the third one the Wellness Center has received, and combines several program elements developed through earlier grants and activities.
\end{abstract}

\section{Program History}

In 1995, Crozer-Chester Medical Center responded to a request from the Chester Upland School District to create a schoolbased health center in order to address adolescent health issues that were impacting the ability of the student body to learn, such as interpersonal violence, chronic health issues, and teen parenting. Though physically located in Smedley Middle School, the Wellness Center serves the entire Chester Upland School District and Chester community. Medical care was made available not just for the students of that middle school, but also for all youth in the neighborhood. Professionals associated with the clinic wanted to incorporate a prevention focus, and took the time to do a study to understand how the youth preferred to learn and what messages they were most likely to listen to. The answer came down resoundingly on the side of learning from peers (as opposed to adults), so staff set out to develop a peer-based curriculum. Their first grant, CATCH, came from the American Society of Pediatrics and focused on 6th graders.

The prevention efforts of the Wellness Center have evolved over the years to reach their present status. The program began with peer leaders (high school students) doing presentations to 6th graders; 7th and 8th graders were added in response to assessments indicating that the effects of 
interventions with 6th graders wore off with time. Prevention work also spread from its original middle school to all middle schools in the school district. Program goals include increasing developmental assets, having a strong sense of self in the present and for the future, serving the community, and refraining from risky behaviors that could threaten the first three goals.

The nature of the peer leader involvement also evolved, incorporating increasing levels of community service, a focus on developmental assets, and developing ambitious but realistic plans for the future. Over the years the peer leader role has become "hot;" youth see it as a desirable thing to be, and the 40 or so peer leaders in each year become role models for middle school youth. They carry the responsibility for conveying the prevention curriculum's content in many venues (in-school, after-school, and community groups), and also for embodying prevention and youth development premises in their own behavior.

\section{Program ANd SERVICE COMPONENTS, AND PARTICIPANTS}

The PL 101 program has four components, the peer leaders themselves, after-school, in-school, and community.

- Youth Leadership Program (peer leaders): about 40 high school youth in grades 9 through 12 are selected for their leadership potential from among those who completed earlier parts of the program, self-referrals, and other referrals. They go through a sevenweek training every summer to learn the curriculum and practice delivering it in ways that will be heard by youth. They deliver the program throughout the school year, and also participate in weekly meetings to discuss issues in program delivery. They also participate extensively in community service.

- Leadership 101 (after-school program): Peer leaders deliver the program after school for about 30 youth in each of three middle schools, grades 6 through 8, who are now calling themselves "junior peer leaders." Every middle school student is eligible; participants are chosen from among those who self-refer or are referred. About twice as many youth (about 60 per school) sign up for the program as eventually participate consistently. In addition to "the curriculum," Leadership 101 provides youth with opportunities for training, service learning, and enrichment activities, but on a smaller scale than for the peer leaders. The program goes throughout the school year, approximately 36 weeks. Sessions last almost two hours with a 15-minute break.

- In-School: The PL 101 coordinator recruits teachers in the school district's four middle schools and one charter school who will make their classes available to the peer leaders. The peer leaders deliver the program to a particular class once a week for 8 weeks, then move on to other classes. The program thus reaches several hundred middle school students each year.

- Community: Peer leaders are invited to do presentations in many venues around the community, including youth-serving organizations and civic groups. 
Parents are involved in PL 101 in a number of ways. At a minimum, they must sign a contract allowing their child to attend the after-school and peer leader components. "Parent events" occur throughout the year; participation varies by school, with school characteristics such as ease of access and sense of community playing a large part in determining the level of participation (e.g., high for a small school where all the youth have gone through each grade together; low for a large school to which youth are bussed from many neighborhoods).

\section{Key Characteristics}

of Participants (Members of

Leadership 101 Only)

\section{Percent}

\begin{tabular}{|l|l|}
\hline Race / ethnicity & $\begin{array}{l}\text { Black: } 97.4 \\
\text { White: } 0 \\
\text { Hispanic: } 2.5\end{array}$ \\
\hline Native English speakers & Most \\
\hline Ages & $12-14: 76.4$ \\
& $15-18: 23.6$ \\
\hline Low-income (200\% of poverty) & Not Ascertained \\
\hline Sexually active & Not Ascertained \\
\hline
\end{tabular}

\section{Curricula}

Train the Trainer for Peer Educators. Currently, training for peer leaders consists of 55 hours of core training, which occurs during the summer. In order to become full peer leaders, participants must meet basic requirements, such as regular attendance and turning in report cards, and must pass certification tests in four areas: public speaking, HIV/AIDS, contraception, and sexually transmitted infections. Returning peer leaders take part in training each year, as a refresher course, often helping to lead the training workshops. Training modules include (1) Orientation, (2) Civic Engagement, (3) Adolescent Sexuality, (4) HIV/AIDS, (5) Contraception, (6) Sexually Transmitted Infections, (7) Alcohol, Tobacco, and Other Drugs, (8) Lesson Plans, (9) Public Speaking, (10) Effective Youth Leadership, (11) Practice Teaching at Local Summer Camp, and (12) Closing Celebration.

After-School Curriculum. The Leadership 101 curriculum is a compilation of activities from curricula on the market and those created by Wellness Center staff and peer leaders. Use of "compilation curricula" has produced in-school programs that are popular with middle school participants, and also effective in terms of evaluation outcomes. The Wellness Center has a curriculum committee of peer leaders that help to create and revise lessons to ensure that they are culturally appropriate, current, and "cool." The Leadership 101 curriculum, as is the case with all program materials, has been reviewed by OAPP to ensure consistency with grant terms and conditions. 
In-School Curricula. The basic 6th grade "Peer Leader Program" curriculum is a compilation from multiple curricula on the market and activities created by Wellness Center staff and peer leaders. The curriculum has been reviewed by OAPP staff to ensure consistency with grant terms and conditions. The Wellness Center has also developed new curricula for the 7th graders ("Think It Over") and 8th graders ("Rites of Passage"). Peer Leaders deliver the curriculum contents to the 6th graders, but not as "lessons" that would feel like school. Instead, the summer training sessions and weekly meetings during the school year he lp peer leaders to convert the major knowledge and attitude contents of the curriculum into games, skits, and other mechanisms that middle school youth will enjoy doing. When there is money to cover 7 th graders, the peer leaders also do that. The 8th grade in-class program is covered by two staff people, when funding allows. It was offered for five years, but is not being offered at this time.

Issues covered in the curriculum include a primary emphasis on building self-esteem through public speaking, civics and community participation, decisionmaking skills, role playing, conflict resolution, and taking leadership with respect to oneself, one's family, and one's community. Abstinence messages are broadly framed as "you can't get where you want to go if these things get in the way." "These things" include sexual behavior, STIs, violence, and substance use and abuse. The realities of local values and cultures are incorporated into the ways the teaching mechanisms incorporate the issues.

\section{Collaboration}

Major collaborative relationships exist between the Wellness Center, the Chester School District (the site of much program activity), and Widener University (for the evaluation), specifically for PL 101. The Wellness Center also connects to every organization in the community focused on youth development through its memberships in the Chester Youth Development Task Force and the Delaware County Pregnancy Prevention Coalition.

\section{STAFF}

PL 101 has one full-time coordinator, one part-time program manager, and part-time support from the Wellness Center director. Three additional staff members assist on a limited basis with large events and activities, or with special projects.

\section{FUNDING}

All of the youth development aspects of the Wellness Center are and have been funded by grants, with Crozer-Chester Medical Center and others providing in-kind services. In addition to OAPP, the center has support from the American Academy of Pediatrics, the March of Dimes, the United Way, the Independence Foundation, Lumina Foundation, and the William Penn Foundation. The Wellness Center is now in the middle of its third OAPP grant. Before receiving its first OAPP grant the center had a small grant from the American Academy of Pediatrics; other grants have followed, but OAPP funding has always comprised the majority of center funding. All PL 101 services and activities are free to youth, and the peer leaders themselves are paid a stipend for the time they contribute during summers and school years. 


\title{
TEACHING RESPONSIBLE ACTIONS IN LIFE (TRAIL), PUBLIC HEALTH AUTHORITY OF CABARRUS COUNTY- KANNAPOLIS, NC
}

\author{
AUSPICES \\ The Teaching Responsible Actions in Life (TRAIL) program operates out of the Public Health \\ Authority of Cabarrus County, North Carolina, which belongs to the umbrella organization \\ Cabarrus Health Alliance (CHA). CHA was created in 1977 and has served the public health \\ needs of the Cabarrus community since its inception. The AFL grant that supports TRAIL is the \\ biggest one CHA has received. Other funding support is either in-kind or in the form of \\ scholarships for participants.
}

\section{PROGRAM History}

The TRAIL program provides comprehensive pregnancy prevention services to interested students attending Kannapolis Middle School (mostly 7th graders). The Cabarrus Health Alliance (CHA) in partnership with the Kannapolis City School District administers TRAIL. The program offers a holistic approach to instill healthier attitudes and encourage abstinence from sexual activity. It provides an abstinence education (AE) curriculum and free enrichment activities designed to empower students toward abstinence.

Upon receipt of the AFL grant in 2002, CHA examined data to decide which school to target in the county and conducted focus groups with parents to get feedback on program design. CHA also conducted in-service programs to school faculty and counselors to get buy-in for the program and to train them on how to identify youth for the program. They implemented TRAIL in Kannapolis Middle School in September 2002 because it is the feeder school to the high school with the highest pregnancy rate in the county-five times the rate of other high schools. The first cohort of students was enrolled for year 1 of the grant.

Youth voluntarily participate in TRAIL's program components. It is a graduated process, starting with the AE sessions and then enrichment activities (which include community service) and tutoring sessions (if necessary). There is also a Summer Advantage Program, which consists of enrichment activities. Approximately 140 students participate each year, with about 5 dropping out of the program annually. Not all students participate in all programming.

The primary goal of TRAIL is increasing youth's intention to remain abstinent. Other goals include (a) increasing knowledge related to sexuality and reproductive health; (b) increasing effective communication and negotiation skills; (c) achieving at least one personal life goal; and (d) increasing self-esteem and self-awareness. TRAIL is guided by its philosophy that participants and peer mentors could build on their assets to improve outcomes. These assets include positive reproductive health, morals and values, presentation and communication, team building, resistance and decisionmaking, and goal setting. 


\section{Program ANd Service Components, AND Participants}

The school counselor and other faculty recruit students into the TRAIL program. Parents and students can also refer youth into the program. TRAIL is not meant to be a program for only "high-risk" or vulnerable youth. Thus, students are recruited across a variety of peer groups and school activities (e.g., youth who participate in athletics or the music program.). The team makes an effort to include a diverse group of youth - based on ethnicity/race, socioeconomic status, peer groups, and school activities-during each set of AE sessions. The philosophy is that this approach will increase the "normality" of going to the TRAIL program and increase the communication across groups in the school. No eighth graders are recruited during the spring semester because they will not have time to participate for a full year before they graduate and move on to the high school. Sixth and seventh grade participants can continue to participate in the program for an entire school year. Recruiters and program staff do not mix sixth grade students with eighth grade students.

The TRAIL program has six components (all of which are voluntary): AE curriculum, enrichment activities, tutoring, the Summer Advantage Program, peer mentorship, and parent involvement.

- AE curriculum (after-school): TRAIL's AE sessions are a modified, shortened version of the widely used Sex Can Wait curriculum. The sessions are delivered after school and are repeated every five weeks for a new cohort of participants. They are designed to empower students with the skills and knowledge to remain abstinent. The sessions are implemented separately for boys and girls, and high school peer mentors participate in conducting the programs along with staff. Students who attend 9 out of 10 sessions receive a $\$ 25$ gift card and those who attend 8 sessions are eligible to attend an End of the Year Social Party. All students receive a certificate of completion and TRAIL t-shirt.

- Enrichment activities (after-school): Once students complete the AE sessions, they advance to the enrichment activities, which are offered throughout the school year. Activities include guest speakers (on topics such as HIV/AIDS and other sexually transmitted infections), community service, and interactive group discussions. As an incentive, food and drinks are provided at every enrichment activity and drawings for gift card prizes are also offered. These activities are designed to allow students to discuss goals and various social pressure issues with their own peers and with high school peer mentors.

- Tutoring (after-school): TRAIL students are also able to participate in the program's free tutoring sessions offered once a week after school. A middle school teacher oversees the tutoring session and high school peer mentors are available to help youth one-on-one. Students are required to work on school assignments or read a book during these sessions.

- Summer Advantage Program: The Summer Advantage Program is also offered at no cost. CHA/TRAIL has partnered with local community-based organizations (CBOs) to offer activities to interested youth. CHA and the Boys and Girls Club of Cabarrus 
(B\&GCC) had a relationship prior to the AFL grant. For this grant, B\&GCC offers TRAIL students scholarships to participate in Camp Spencer, which is a summer day camp that provides activities such as swimming, canoeing, arts and crafts, fishing, and sports. It is intended that the most well-behaved students in the program during the school year will participate in Camp Spencer. Year 3 (Summer 2005) will be the first time that Camp Spencer is coed. Unlike other sponsored activities, buses to and from the camp are provided. The American Red Cross also collaborates with CHA by providing HIV/AIDS counseling/information sessions to TRAIL students, as well as an eight-hour "Safe Sitter" Course, a babysitter training to provide youth with the knowledge and skills of safety, responsibility, and leadership. Arts Experience is another local CBO providing summer scholarships to TRAIL students to participate in their summer art programming.

- Peer mentorship: The high school peer-mentoring component consists of about 17 trained juniors and seniors who become the liaisons for the middle school aged youth. These peer mentors are recruited countywide, not just from the high school that TRAIL students will ultimately attend. TRAIL program staff train the peer mentors for five weeks on HIV, general health education, asset building, and presentation techniques. Peer mentors participate in all TRAIL program components, based on their availability and interest in the activities. Peer mentors receive points for every activity they attend to encourage ongoing and frequent participation in the program. The points allow them to earn prizes, including an annual trip (e.g., this year the trip is to the Outer Banks).

- Parent involvement: The parent component was not a major focus of the program, however, the new project manager has strengthened this portion of the program. Parents are encouraged to actively participate in TRAIL. They must sign a parental consent form for their child to participate in the program. The AE sessions include take-home activities for families and participants to engage in together. Other components include periodic parent workshops and student/parent monthly newsletters. Parents have also been encouraged to attend appropriate enrichment activities and occasionally a parent or parents will do so, particularly programs in the community.

\section{*TRAIL did not have the data necessary to fill in the Key Characteristics of Program Participants table.}

\section{CURriculum}

TRAIL's AE sessions are a modified, shortened version of the widely used, state approved Sex Can Wait curriculum. The original curriculum is not used in its entirety because the program has found that it is not effective with males. Also, the original curriculum required too much student writing and not enough interactive discussions. The original curriculum consists of 24 sessions, but TRAIL condensed its curriculum to 10 two-hour twice-weekly sessions while still covering all topics. The students are required to complete weekly a 10-minute take-home activity with their parent(s). Topics covered include self-esteem, values, dating, peer pressure, media influence, puberty, communication, healthy relationships, goal setting, and sexual decisionmaking. These educational sessions are designed to help students establish realistic goals 
and attain them. These sessions are delivered after school and are repeated for a different cohort of participants every five weeks. The classrooms are single sex and are led by a certified health educator. One or two peer mentors are also present in each classroom to supplement and colead the sessions. The method of teaching varies by topics-role-plays, small group discussions, presentations, and guest speakers.

\section{Collaboration}

The primary collaborative relationship exists between the Cabarrus Health Alliance and the Kannapolis City School District. School staff members recruit TRAIL participants, activities are implemented on school grounds (including the AE curriculum, tutoring, and some enrichment activities), and a teacher supervises the tutoring activity. Other collaborations exist with the Boys \& Girls Club of Cabarrus, the American Red Cross, and Arts Experience for the Summer Advantage Program. Staff members from UNC-Charlotte are the evaluators on the project.

Healthy Cabarrus (HC) has also had a long-standing relationship with CHA before the AFL grant. This CBO formed a teen task force of 20 high school students from each local area high school. Each group focuses on one topic per year and attempts to resolve the issue. HC also provides student volunteers to schools where needed.

\section{STAFF}

TRAIL has two health educators (one full-time and one part-time) and part-time support from the CHA director. About 17 peer mentors volunteer for the program. One peer mentor is paid throughout the summer to continue participation. The evaluation team receives a small amount of funding for its work.

\section{FUNDING}

TRAIL is primarily funded by the AFL grant. In addition to OAPP, CHA has support from the Boys \& Girls Club of Cabarrus, Red Cross, and Arts Experience in the form of scholarships to the Summer Advantage Program. All TRAIL services, activities, and incentives are free to youth, and the peer mentors are given incentives throughout the year. Once the AFL grant ends, the sustainability of TRAIL is not promising. With the closing of Pillowtex (a local manufacturer), many community resources are going toward those who have been unemployed as a result of this industry shutdown. Thus, funding for public health needs is very limited.

\section{THE CHILDREN'S COUNCIL-LANCASTER, SC}

\section{AUSPICES}

The Children's Council is a private nonprofit organization that started as a coalition in 1987 and incorporated in 1989. The Children's Council was originally named Healthy Mothers, Healthy 
Babies. Its operations began as a result of a dramatic increase in teenage pregnancy in this small and religious town.

\section{PROGRAM History}

The local governor's council ${ }^{10}$ conducted a "Communities that Care" youth survey process and prioritized the top needs of the community. They found that when youth get pregnant, they drop out of school, so efforts target keeping youth in school. The Children's Council began focusing its teenage pregnancy prevention efforts in one Lancaster County high school using state money (for five years). With the AFL grant from OAPP, it has been able to expand its efforts to all four county high schools.

Upon receipt of the AFL grant in 2002, the Children's Council (and all subcontractors) implemented its program in the middle and high schools throughout Lancaster County. The stated primary goal of the program is to reduce the teenage pregnancy rate in the county via six components. Many of their efforts stem from the long history of research done by Doug Kirby, J. David Hawkins, Richard Catalano, and Lynn McDonald.

\section{Program ANd Service Components, AND PARTiCiPANTS}

According to program statistics, during the period of July 1, 2003 through June 30, 2004 (year 2), 546 female and 588 male clients participated across all components of the program. The client pool was predominantly Caucasian and African-American youth and high school-aged. Almost 100 parents (mainly mothers) attended the program in some capacity.

The Children's Council has six components that interconnect abstinence education with youth development strategies.

- Peer Refusal-Skills Training (in-school): Led by the Children's Council, all 9th grade students in Lancaster County receive an eight-week school-based health curriculum derived from the nationally recognized Sex Can Wait curriculum. Approximately 16 sessions are held in each high school with an average of 35 students attending per session. The classrooms are coed. The primary objectives of these educational sessions are to increase the knowledge of healthy reproductive behavior among the students and to arm them with the skills (i.e., peer refusal) to make appropriate decisions regarding abstinence. The Children's Council staff conducts eight sessions, while the health teacher conducts the rest. The Children's Council staff cannot entertain discussions about birth control or abortion. However, teachers are able to answer questions about birth control. One part of the curriculum is the Baby-Think-It-Over technology that realistically demonstrates the impact of teenage pregnancy through the use of an infant simulator. This component also increases parents' awareness of the impact of teenage pregnancy. A parent consent form is required for participation in Baby-Think-It-Over.

\footnotetext{
${ }^{10}$ Governor's councils are local organizations started during a previous administration. Localities across the state started governor's councils to keep youth out of the juvenile justice system and social services. Lancaster's council includes a prevention arm.
} 
- Families and Schools Together (FAST) (evening): Led by Communities in Schools, FAST is a nationally recognized research-based family-strengthening program designed to promote family communication, reduce conflict, and increase support to isolated rural families. Middle school students in all four schools and their parents are eligible to participate. Home visits are also done as part of recruitment. Incentives are given to get the parent consent forms back. About 15-20 families participate per site, totaling 70 families per school year. Families and their middle school child come weekly for 10 weeks, and activities are conducted in the school cafeteria. The participating children meet first for four weeks prior to the family sessions. The Lancaster program has graduated at least 10 families at each of its four sites. An important factor in the success of this program, in the view of project staff, is the consistency of the staff and their home visits. Project staff is racially diverse to reflect participating families.

- Academic Assistance Lab (after-school): This component occurs Monday through Thursday in one-hour sessions after-school in all four county high schools, with approximately 3,300 participants. It is reported that a large portion of the students come regularly. The lab's objective is to reduce school failure and promote attachment to school. A gift card incentive exists to increase participation. Bus transportation is provided from the assistance lab for students attending Lancaster High School (the county's largest high school).

- LearnTV (in-school): This component is a semester-long class-based production done by students in Lancaster High School and led by the Lancaster County School District. It targets the entire community through cable TV and strives to increase favorable attitudes toward teenage pregnancy prevention, as well as other pressing community issues (such as substance use during pregnancy and postpartum). The class produces programs on topics of interest and public service announcements. These classes meet five times a week and are 1.5 hours long. Two sessions are held each day. Attendance in the classes is dependent on youth's school attendance since this is an in-school course.

- Can We Talk (evening): Led by the Children's Council, this is an interactive parent workshop that helps parents enhance their role in their children's health and sex education while respecting the values of the family. These workshops take place in the evenings in churches, businesses, and schools.

- Brother to Brother (after-school): Led by the Lancaster Fatherhood Project, this component is a curriculum-based youth mentorship program employing college and high school students to facilitate male leadership and responsibility, peer refusal skills, and sound decisionmaking skills. The target population is African-American middle and high school boys, and the program is held in a community center four times a week after school. 
Key Characteristics

of Participants Percent

\begin{tabular}{|l|l|}
\hline Race / ethnicity & Black: 42 \\
& White: 56 \\
& Hispanic: 1 \\
\hline Native English speakers & All \\
\hline Ages & 12 and under: 6.3 \\
& $13-14: 74.8$ \\
& $15-17: 11$ \\
& 18 and above: 0 \\
\hline Low-income (200\% of poverty) & Not Ascertained \\
\hline Sexually active & Not Ascertained \\
\hline
\end{tabular}

\section{CURriculum}

As described above, the Children's Council, along with school health staff, implements the Peer Refusal Skills Training educational sessions. This group is dedicated to implementing programs that have been evaluated and are considered best practices. It also focuses on the fidelity of the implementation of the program to ensure similar outcomes to previous studies.

\section{Collaboration}

The community-wide collaboration is extensive and all youth-serving agencies are represented on the local Governor's Council. As a council, the agencies identify problems of focus, create requests for proposals that will provide money to address the problems, and identify the most appropriate agency to conduct the program. The council works on the proposal as a collaborative group. Multiple responders noted the spirit of collaboration existing in Lancaster County and very little turfism.

For this grant, the major collaborative relationships exist between the Children's Council (which takes the lead in delivering the Peer Refusal-Skills Training educational sessions-Sex Can Wait and Baby-Think-It-Over-and Can We Talk), Communities in Schools (FAST), the Lancaster County School District (access to health classes for the Peer Refusal-Skills Training; Academic Assistance Lab; Learn-TV), and the Lancaster Fatherhood Project (Brother to Brother). Communities in Schools is located in the same building as the Children's Council.

\section{STAFF}

There are three certified FAST trainers employed by the Children's Council and two certified trainers employed by Communities in Schools. FAST also hires a parent partner-a former parent participant from the community-to colead the groups. Two educators conduct the Peer 
Refusal-Skills Training educational sessions along with the health teachers who are employed by the Lancaster County School District. Children's Council staff also implement the Can We Talk parent workshops. High school teachers staff the Academic Assistance Labs, the Lancaster School District supplies the instructor for LearnTV, and the Lancaster Fatherhood Project provides staff for the Brother to Brother component.

\title{
FUNDING
}

Prior to the OAPP grant, the Children's Council had been focusing its teenage pregnancy prevention efforts in one Lancaster County high school using state money. This grant has enabled them to expand their efforts in all four count y high schools. The FAST component also receives funding from state and local agencies.

In the future, the Children's Council is likely to seek funding from the Lancaster County School District and local foundations as a result of its growing reputation in the community. This will be particularly helpful for sustaining the FAST and Peer Refusal-Skills Training components, which are their most successful components of the overall project, according to the pre- and posttests conducted during these programs.

\section{FAME (FAMILY ACTION MODEL FOR EMPOWERMENT), BETA CENTER- ORLANDO, FL}

\author{
AUSPICES \\ BETA Center is a private, nonprofit agency offering comprehensive support services that address \\ the special needs of youth, parents, and families. BETA Center, founded in 1976, offers a \\ residential facility, counseling, an alternative public school for pregnant and parenting teens, on- \\ site child development center, emergency food and baby pantry, neighborhood based parenting \\ education classes, youth development services, and other educational programs targeted to \\ families in the central Florida area. The Family Action Model for Empowerment (FAME) \\ prevention project operates out of BETA Center's Neighborhood Services.
}

\section{Program History}

BETA Center began by opening a private shelter equipped to meet the needs of homeless pregnant teens, and quickly thereafter established a GED program onsite. BETA Center became a well-known and respected agency in the central Florida area and was soon approached to implement youth development programs in the local public schools and community settings. BETA began its youth development initiatives with conflict resolution, anger management, and self-esteem classes designed to help elementary school age youth and adolescents make more positive decisions and regain a positive outlook for their futures. It was a natural progression for BETA to add an abstinence component to their self- esteem program, so when it received OAPP funding BETA revised its existing curriculum to include abstinence messages and goals. The 
new program was named the Family Action Model for Empowerment (FAME) and became known as the "prevention arm" of the BETA Center.

FAME is an integrated approach to working with families through providing community-based services that bring support, education, and resources to parents and children in high-risk and economically disadvantaged neighborhoods. FAME is the prevention component of the BETA Center, providing services to promote sexual abstinence and positive choices among at-risk adolescents. The primary service area of FAME includes two zip codes in Osceola County that are documented as having the highest rates of teen births in that county. FAME's primary goal is to help children improve and increase their level of communication with their parents. FAME also hopes to influence adolescents in a positive way by teaching them to remain abstinent from sexual activity until marriage. FAME attempts to achieve these goals through interventions aimed at both children and their parents, working with adolescents and parents separately and together to build trust and communication.

When FAME began, it worked in about two schools a year, and now is up to 5-6 locations a year (elementary, middle, and high schools) and various apartment communities, although these are not always the same schools from year to year. In 2004, FAME provided services to 761 youth and parents through Prevention and Abstinence Projects and to an additional 371 youth and parents through other Family Fun events. Some children are referred to the FAME program by their schools while other children opt into the program voluntarily.

\section{Program and Service Components, ANd Participants}

- Youth component: FAME offers self-esteem and abstinence education interventions to youth in 5th through 9th grades at community centers, churches, schools, apartment complexes, and other locations in the target area. Led by BETA Center staff, children participating at a given location are organized into groups of 15-25 students and receive 50-60 minute self-esteem and abstinence education sessions once a week for 10-12 weeks. Larger groups may have two facilitators (they try to keep the ratio to about 15 children per facilitator). The skills developed in these sessions include problem solving, decisionmaking, creative self-expression, goal setting, building healthy relationships, critical thinking, value definition, self-development and identity formation, and resistance skills. These skills are developed through the use of self-esteem curriculum, conflict resolution discussion and workshops, music classes and performances, and performing arts instruction and events. Abstinence education messages are intertwined in every session, whether a curriculum-based class or a music lesson.

- Parental component: Parents participate in workshops designed to address parent-child communication, prevention of risk behaviors, and other parenting topics. In one year, up to five 9-week parenting cycles may occur with some parents participating in more than one cycle. Childcare and food are provided at all parent workshops and often the youth participate in an abstinence event while the parents receive their training. In 2004, 127 parents attended the FAMEous Parents' series. 
- Child care and tutoring: Childcare is provided at all the Parents' series and older teens are trained and used as tutors. While younger children are in childcare, schoolage children are matched with teens for tutoring focused on reading, literacy, and homework assistance. The tutoring component is provided as the teens are available.

- Other aspects: FAME reaches out to families in a number of ways in addition to its formal youth and parent interventions. FAME plans family and community events to help strengthen relationships, provides information and referrals to families with specific concerns, provides after school activities including tutoring, mentoring, and arts, and the program maintains an advisory council comprised of youth, parents, social agencies, and other community groups to address the needs of the community.

\section{CURriculum}

FAME implements a 12-week youth development curriculum focused on self-esteem and abstinence. The curriculum includes topics such as self-esteem, values clarification, the benefits to abstinence, healthy relationships, making positive choices, feelings and refusal skills, critical thinking and positive conflict resolution skills, self-development, and looking towards the future. FAME's curriculum was developed by BETA Center based upon the past experiences with other, formal curricula. BETA found itself constantly tweaking other curricula and decided to develop its own based upon the needs of the youth they were serving. The current curriculum does have an "It's Great to Wait" component. The parenting curriculum uses some components of the "Can We Talk" program. Both curricula have been approved to use in this project by OAPP.

\section{Key Characteristics}

of Participants

\section{Percent}

\begin{tabular}{|l|l|}
\hline Race / ethnicity & Black: 14.5 \\
& Hispanic: 58 \\
& White: 14 \\
& Asian: 1.8 \\
& Other: 11.4 \\
\hline Native English speakers & Not Ascertained \\
\hline Ages & Under $12: 15$ \\
& $12-14: 62$ \\
& $15-17: 7.75$ \\
& $18-34: 6.7$ \\
& 35 and older: 8.6 \\
\hline Low-income (200\% of poverty) & Not Ascertained \\
\hline Sexually active & Not Ascertained \\
\hline
\end{tabular}




\title{
STAFF
}

FAME supports 3.5 full-time equivalent staff members including a project coordinator/parent educator, two youth specialists, a director of integrated art, and several program support staff members. Support staff and program facilitators, funded by the BETA Center budget, also work on the project.

\section{FUNDING}

In 2004-2005, FAME received 60 percent of its funding from the OAPP grant and BETA Center absorbed the remaining 40 percent of costs for the program. (The program also received some money from United Way and community donors, although this money will not be readily available in 2005-2006.) In the upcoming year, BETA Center will be covering 50 percent of the costs to run FAME and the OAPP grant will cover the remainder.

\section{FIFTH WARD ENRICHMENT PROGRAM-HOUSTON, TX}

\begin{abstract}
AUSPICES
The Fifth Ward Enrichment Program Incorporated (FWEP) is a youth leadership and development program for at-risk males, ages 12-19, located in one of Houston's most economically depressed neighborhoods. FWEP is a local private nonprofit agency that is also a United Way Affiliate organization located within Houston's Fifth Ward, the geographical area for which it provides services. FWEP is designed to empower boys to become responsible men and productive members of their families and the Fifth Ward community.
\end{abstract}

\section{PROGRAM History}

Rudy Wagner and current chief executive officer Ernest McMillan founded FWEP in early 1984 in response to the community's skyrocketing rates of teenage pregnancy, crime, school dropout, drug use, and a lack of responsible African-American fathers.

FWEP grew out of the Urban Affairs Corporation (UAC), which was founded in 1979. This organization attempted to free women of childcare responsibilities so they could be more engaged in society and participate more in the world through education and employment. The main concern of UAC was women becoming parents at too young of an age, which led the organization to develop a school-based clinic (one of the first in the country) in one of the Fifth Ward's high schools. The missing element in the focus on reducing teen pregnancy was the role of young men. Ernest McMillan was brought on board to figure out how to get men involved in the UAC's mission and projects. His involvement began with a concept paper that eventually led to the creation of the FWEP. 
The Fifth Ward is one of Houston's more economically distressed neighborhoods. The FWEP attempts to help the neighborhood's young men redirect their energies into positive goals for the future rather than falling into the destructive path taken by many youth. Males acquire social and leadership skills through male role models, mentoring techniques, academic support, and therapeutic activities. In addition, they learn the importance of respect and communication in their relationships with others, specifically women.

The FWEP began with a pilot program offering int erventions to 356 th and 7 th grade boys who were the most problematic and difficult cases in the community. The program first ran 5 days a week for 8 weeks during the summer of 1984, and offered life skills, education, field trips, and so on. The young men came to the program relatively enthusiastically because some of the activities were fun and recreational (like fishing and swimming).

The young men appeared to accept the program, and that appearance carried over into the school year. Teachers in the local school from which the young men were recruited filled out surveys to explain their needs. FWEP met with the principal from the source school and continued working with the school during the school year. FWEP began offering after school activities, weekly education workshops with parents, community activities, in-school counseling, and other activities.

\section{Program AND Service COMPONENTS}

FWEP places special emphasis on social and therapeutic enrichment. FWEP focuses on creating strong ties between the young men and "caring people to whom they can go with their problems." A large component of FWEP is reshaping self-images through positive identity formation. In addition, community service and service learning are used as development tools.

The program is located in three local high schools and two local middle schools, each of which has a project manager. Every student gets life skills twice a week (approximately). This intervention usually happens somewhere away from school so the kids are more relaxed and willing to talk freely.

- In school: During homeroom and lunch periods, the project manager in each school is available to talk with FWEP participants from his school about life skills or abstinence issues, through small groups or individual counseling sessions. Some of these are scheduled but many are spontaneous.

- After school: The project manager meets with FWEP participants when classes end in assigned classrooms or prearranged school sites. After signing in, the project manager either delivers the day's planned indoor activity (life skills, guest speakers, current events, snacks, etc.) at the school site, or takes the students by agency van to another approved location for multiple activities (indoor and outdoor.) The after school activities might include field trips, outings to museums or other cultural events in Houston, recreational activities like basketball or sports, and a variety of life skills, interpersonal 
development, and abstinence education events. Often, the boys discuss abstinence and other youth development issues with their project manager while in the van on the way to the after school activity site. Other times, the project manager might take small groups out for counseling, rap sessions, snacks or small meals.

\section{- Examples of activities:}

- Peer Health Educators-6 young men who commit for the whole school year to present information through skits about abstinence, health, and other topics kids don't talk to their parents about. The boys watch the skits and then participate in conversations.

- Teen Enterprise Center-This center attempts to teach the young men about business by having them work on real projects. Some of the projects include tshirt printing, computer repair, film production and editing, mural arts, and photography.

- Youth are taken to local Houston universities to work with current college students. This activity is used to generate interest in attending college and to show the young men that attending college is an attainable goal.

- Cultural Arts Events-The young men go to museums, (Shrine of the Black Madonna Cultural Center, the Buffalo Soldiers Museum, the Children's Museum, the Museum of Fine Arts), music, art, and other cultural events. FWEP wants the kids to connect with their heritage and to look at accomplishments of other black individuals.

- Career Shadowing Day_FWEP collaborates with many companies and agencies to expose the youth to the "real workplace". Youth are matched with male volunteers, working adults (professional, blue collar, doctors, law enforcement, etc.) and spend a day with that individual at his job or worksite. When individual, one-on-one matches can not be met, FWEP will arrange for small groups of youth (3-6) to be transported to a host company that will chaperone and engage the group regarding the variety of jobs, company history, family relations, and the like. Many of these adult volunteers are former FWEP staff members, past and current mentors, board members, and FWEP alumni. Few are residents of the Fifth Ward area and many are non-Hispanic or African-American.

- Former residents of the Fifth Ward come to the schools to talk about how they got to where they are in their careers. They often talk about their educational background and about what the students want to do in the future. These guests talk about the adversities and barriers they have overcome in their careers, what they like about their jobs, how much money one can earn, and so on.

- All Males Day-This day involved an assembly in the school for the boys. The young men listen to their teachers and project managers talk about their experiences and backgrounds. This allows the boys to see their teachers and project managers in a different light and to ask questions about the transitions of "boys to men" and school-to-work 
- Summer: Historically, FWEP provides each youth in the seven week summer program with a stipend of $\$ 10$ per day, $\$ 350$ total, not including fines or bonuses. FWEP pays $\$ 10$ per day of completed work at an internship.

Parents are involved in FWEP mostly through consent forms and informal conversations. The project managers communicate with the parents over the phone and in person at least on a monthly-basis. FWEP staff express concern about the difficulties of involving parents. FWEP has a designated staff member who is responsible for parent-program communication (Ms. Manning, the parent liaison). Not many parents attend FWEP events but project managers do see parents on a fairly regular basis when dropping off the young men in the evenings. Parent participation increases when FWEP events offer food or raffle opportunities. Middle school parents are much more involved in FWEP than high school parents.

\section{CURriculum}

FWEP uses Sex Can Wait, the abstinence-only education adopted in response to OAPP's requirements. FWEP also uses a violence prevention curriculum to teach the young men not to participate in domestic and community violence. The curricula are delivered to all participants of the FWEP in informal ways, through conversations after school, so the intervention does not feel like school. Abstinence messages are not limited to sex but also include alcohol, drugs, violence, bad language, bad clothing, and the like.

FWEP's attitude towards its participants is: "a student is not doomed because he had sex, but he can be a better person and recommit to a life without negative behaviors. We will help him do so." FWEP strongly believes that everyone makes mistakes but that those mistakes can be corrected. FWEP does this by helping to create strong bonds between the young men and caring people in the community who can help the boys with the challenges they face. FWEP focuses on personal development, safe choices for healthy living, interpersonal development, "our heritage", and exploring life careers. A central aspect to the curricula is goal setting and goal attainment.

\section{Collaboration}

FWEP has created many partnerships and collaborations with many different organizations in the Houston area:

- Houston Independent School District: the HISD is FWEP's most far-reaching collaborative partner. FWEP has a contractual agreement with the Houston Independent School District. HISD allows FWEP to establish project sites at any of its northeast district schools. Currently, FWEP has sites at five schools in the district. Each year, FWEP briefs the HISD on the upcoming year's events and activities and the HISD shares its expectations for the program. HISD allows FWEP to maintain offices in four schools in the Fifth Ward.

- Communities in Schools (CIS): FWEP partners with CIS and, at some sites, shares office space with the CIS rep and does extensive cross-referrals with CIS. CIS tends to handle the school and employment-oriented needs while FWEP serves the mentoring and enrichment needs of youth. In addition, FWEP organizes joint activities with CIS that include enrichment activities. 
- Junior Achievement: Junior Achievement is an organization that promotes entrepreneurship, economic literacy, and so on. This organization links up with FWEP during the summer program to help teach economic and business skills to the young men. Exchange City is a program in which kids learn how to run their own city. It operates like a simulation where the young men elect their mayor, police chief, and the like. and they have their own newspaper, cafeteria, stores, and radio station. The young men learn enterprise skills in a fun and experiential environment.

- The City Health Department, which has clinics in the Fifth Ward area, assists FWEP by providing health screening and other health services.

- FWEP has also developed a close relationship with the University of Texas School of Public Health because this school does the program evaluation.

- Urban Scouts: FWEP has placed some of its students in Urban Scouts, a program that acts like the Boy Scouts for lower-income, urban youth and has fewer restrictions than boy scouts (no uniform is required and it has less financial burden).

- People in Partnerships: A community builders program. Through "Youth Cadres" People in Partnerships' youth help train FWEP youth to build stronger communities.

- Voices of the Future: This organization is planning a youth retreat in May. Adolescents speak and adults listen. Adults in the community are trying to be tolerant of youth by learning to respect the rap music, for example, and Voices of the Future has collaborated with FWEP to work together on this issue.

\section{PROGRAM PARTICIPANTS}

Currently, FWEP engages about 1,000 12-19 year old young men per year through all of its interventions. Of these, 225-250 young men are served on an individual caseload basis. The project managers, along with their peer mentors, work with their assigned caseloads of students daily. The rest of the 1,000 kids receive some sort of outreach service or intervention. About 200 individual caseload participants are African-American and the rest are Hispanic.

Key Characteristics of Participants

Percent

\begin{tabular}{|l|l|}
\hline Race / Ethnicity & Black: 80 \\
& Hispanic: 20 \\
\hline \multirow{2}{*}{ Ages } & $11-12: 21$ \\
& $13-18: 78$ \\
& Above $18: 1$ \\
\hline Low-income (200\% of poverty) & Under $\$ 5,000: 11$ \\
& $\$ 5,001-\$ 10,000: 57$ \\
& $\$ 10,001-\$ 25,000: 26$ \\
& $\$ 25,001-\$ 50,000: 6$ \\
\hline Sexually active & Not Ascertained \\
\hline
\end{tabular}




\section{STAFF}

The front-line staff includes a lead manager, three project managers who are located at the school sites, a Teen Enterprise Center manager, five peer mentors (college students), several trained peer health educators (high school students), and a parent liaison.

\section{FUNDING}

FWEP receives funding from a variety of sources. About 35 percent of its funding comes from foundations, 35 percent comes from grants, most of which are federal, 15 percent comes from corporate and individual donors, and the rest comes from other assorted donations. FWEP's funding has decreased significantly in the past several years and the program has had to eliminate one middle school from its operations as well as its Young Fathers and Family program, which had been federally funded. The Teen Enterprise Center has also lost foundation funding and has had to cut back on services. All FWEP services are free. The peer health educators and peer mentors are contracted and paid minimum wage during the schoolbased project year. Youth attending the summer program and participating in the Teen Enterprise Center business activities are paid stipends related to their assignments.

\section{INTEGRATION OF ABSTINENCE AND YD COMPONENTS}

The seven AFL-funded programs we visited were chosen deliberately for the extensiveness of their youth development approaches. Our choices proved "truer than we knew," in the sense that all of them completely integrate their abstinence and YD components, making it impossible to achieve one of this project's original goals of determining how to evaluate "the added effects of YD on abstinence messages."

One reason that abstinence education and YD were inseparable in the programs we visited is that most of the programs were doing YD long before they received an AFL grant-either their present one or previous ones. Four programs_-PLP/Portland, Maine; The Real Deal/Brooklyn; FAME/Orlando, and FWEP/Houston—had significant histories of youth development work before they applied for AFL funding. These programs fit the abstinence message into their overall message of "set goals, develop assets, look to the future, you can do it." In these contexts the abstinence message joins other messages about behaviors best avoided because they will make it harder or impossible to achieve longer-term goals. The fifth program-Children's Council/Lancaster County, South Carolina—had a greater focus specifically on sexuality than the other four, having already been working on teen pregnancy reduction with state funding for five years before receiving its AFL grant. However, it was doing this in the context of a future orientation and life planning. Only two of the programs visited-Leadership 101/Chester, Pennsylvania and TRAIL/Kannapolis, North Carolina-began with an AFL grant, and even they deliver their sexual abstinence messages in a broader context of future orientation.

Another reason we are unable to separate the effects of abstinence education and YD had to do with how abstinence messages were delivered. Only three of the programs visited-The Real Deal/Brooklyn; TRAIL/Kannapolis, North Carolina; and Children's Council/Lancaster County 
South Carolina-had program components that could be identified as the primary times and places they did "abstinence education." That is, they had classes or time periods devoted to sexuality education specifically, during which they used common abstinence curricula and generally "stuck to the topic." Even these programs, however, blended the abstinence materials in these sessions with more general YD messages, used YD-type activities to help sell the abstinence messages, and modified standard curricula to incorporate both additional messages and a variety of techniques and activities to make the sessions more "fun" and less "like school." Their major emphasis is on helping kids realize they can establish and achieve realistic and obtainable goals and have a decent future. A variety of experiences succeeding at goals are incorporated into these programs.

The remaining programs visited were even less likely to label anything — an activity, a message, a time period-as "abstinence," or to use common abstinence curricula in anything remotely resembling a school-like manner. Mostly these programs relied on their presenters, whether staff or peers, to memorize the messages, work on fun ways to put the messages over, and be prepared to turn every incident into an opportunity to reflect on implications. For example, staff in more than one program said "van time is the best time," while traveling to and from events with a small group of youth, to take chance remarks and draw youth into a general discussion of what they mean and what they imply for their own preferred behavior. Even the peer leaders in Chester's Leadership 101, who rely most on a particular curriculum for the content they convey, spend their summers learning the curriculum and developing interesting ways to convey it so they can leave the actual curriculum volume behind when they work with children in the afterschool program.

Further, although all of the programs visited identified one or more specific abstinence education curricula that they used, every one of them had also modified these curricula in major ways, as already noted. They used different components with youth of different ages, or they created their own curricula for youth who had been through "the program" one year and were back for subsequent years. Or else they simply made it a point to discuss curriculum points when opportunities arose, and they bent their efforts to making sure the opportunities did occur in the course of enrichment activities.

\section{IMPLICATIONS FOR EVALUATION}

The programs we visited were able to combine YD and abstinence content and approaches in ways that parallel the integrated programs for which evaluations have documented effectiveness (see chapter 2). Even the programs that had identifiable time periods during which abstinence education was the major activity had extensively modified, augmented, and adapted standard curricula with YD approaches to develop the approaches they had found to be most effective in conveying abstinence messages to youth. The messages remained consistent with messages in standard curricula, but most programs made great efforts not to feel like school, with the consequence that books, readings, handouts, "homework," and tests were little in evidence.

We cannot answer the question of whether these programs are more effective than those that do not incorporate YD components or goals, because we do not have the information needed from both types of programs to make this comparison (we recommend in chapter 5 that OAPP develop 
this type of cross-program capability). Program evaluations for the seven programs visited do show improvements in knowledge, attitudes, and behavioral intentions, and in the few instances in which actual behavior is measured, in behavior. But some of these evaluations do not include control or comparison groups, so no strong conclusions can be drawn. We can say that the programs "felt" effective, and that their own strongest evidence is that youth return year after year to participate and do not, in general, "get into trouble" of a sexual or other nature.

Based on site visit findings, we must conclude that for the seven programs we visited, it would be impossible for an evaluation to be designed that was capable of separating the effects on desired outcomes of YD and abstinence components. We have any number of suggestions for improving the evaluations currently under way in these programs, and offer them in the next chapter after presenting an overview of the programs' evaluation designs. But OAPP will have to look elsewhere to be able to assess the value added of YD for abstinence programs. We reserve discussion of these options for chapter 5. 


\section{CHAPTER 5}

\section{EVALUATING ABSTINENCE AND YD COMPONENTS OF AFL-FUNDED PROGRAMS}

The primary goals of this project all revolved around strengthening OAPP's ability to improve, through evaluation, the effectiveness of the programs it supports. We began the project with a review of literature evaluating YD programs, to identify characteristics of YD programs that were most likely to affect abstinence itself or the personal assets that might promote abstinence. We examined evaluation reports submitted by current AFL grantees to learn what they are doing by way of evaluation, analyzing the strengths and weaknesses of their evaluation efforts. We also reviewed year-end reports submitted to OAPP, and assessed what these reports might tell OAPP about program effectiveness. Finally, we conducted site visits to examine how youth development approaches are being incorporated into abstinence education, and whether evaluations might be designed that could detect the separate effects of YD approaches and abstinence education.

This chapter pulls together our findings with respect to OAPP's ability to learn "what works" from evaluations applied to AFL grantee activities, and recommendations for improving that ability. It starts with very simple suggestions for improving the utility to OAPP of the basic information that every project submits every year in the form of year-end and evaluation reports. Then it turns to specific program evaluations, making suggestions for improving the evaluations of the programs site visited and generalizing from these to ways to strengthen evaluations across all AFL programs. It concludes with several recommendations for cross-program evaluation designs that could help OAPP identify its most effective programs.

\section{STRENGTHENING THE USEFULNESS OF YEAR-END REPORTS}

Several changes in AFL grantee reporting requirements could give OAPP much better information from the reports it routinely receives from grantees. We phrase these changes in the form of recommendations.

\section{Revise the reporting requirements and format of the AFL grantees' year-end report to require all grantees to use a similar detailed structure and report certain basic information in a similar fashion.}

The breadth, depth, and overall quality of the year-end reports varied significantly across the AFL grantees. OAPP could be learning significantly more from these documents if they had a consistent format and reliably contained a minimum set of consistent information. Better structure and reporting requirements would make it easier for OAPP to extract program information and compare across grantees. At the same time, these new reporting requirements should not place additional burden on the grantees. 
Appendix E provides a suggested template for the year-end reports. The template is intended to ensure that all of the necessary program information, including that related to positive youth development strategies, is collected, and that it is organized in the same way.

\section{Require programs and evaluators to record and report information about program intensity and average "dosage" received by participants.}

One of the more striking omissions from most year-end and evaluation reports was the lack of information on participation levels. Few reports provided the participation rates in the form of average number of sessions attended, the median number of sessions attended, and the low and high ends of the participation range. Sixty percent of reports provided enough information about how long the program was expected to last and how many sessions were being offered each week, so we could develop a measure of program intensity for these programs. But 40 percent did not provide even this basic information, and far fewer indicated how much program participants were actually exposed to the components of the program. Such participation rates and dosage information are important in understanding whether the program is actually serving the children it intends to serve at the intensity for which the program was designed. This information and can help to assess and qualify any evaluation findings that may emerge.

In addition to requiring programs to record and report participation rates, OAPP should consider the participation statistic or statistics that would be most useful for its purposes. Among those evaluations that reported participation statistics, some reported the number of youth enrolled, others reported the average number of days attended by participants, and still others reported the percentage of participants attending the program every day. Creating a uniform participation measure or series of measures will give OAPP another standardized way of monitoring the progress of its grantees.

\section{Routinely summarize the year-end reports for internal use in assessing which programs incorporate abstinence education and youth development practices for which evidence of effectiveness exists.}

Once reports contain relevant information in a consistent format, OAPP's evaluation staff and project officers should conduct their own annual summary of grantee activities. The common report structure and contents will make it easier for OAPP programs officers and evaluation staff to summarize what each program is actually delivering to youth; classify program activities by program intensity, dosage, and content; identify use of evidence-based practices; and begin to set up a structure for comparing across programs. The result should be a chart (see appendix D) similar to the summary of the document review performed for this evaluation. This review would help OAPP make decisions about how to invest its resources in programs that offer activities already known through evaluation to be effective at delaying onset of first sex. It is also an essential first step in structuring future evaluations to learn more about how youth development activities could enhance the effectiveness of abstinence education programs, which we discuss further below. 


\section{Develop a core set of outcome measures that each program is required to collect.}

If OAPP wants to be able to assess the relative effectiveness of different AFL projects, it is essential that each project measure certain outcomes and that they measure these outcomes in consistent ways. Basic outcomes of importance to AFL grantees are knowledge pertinent to sexual behavior and decisionmaking, attitudes pertinent to sexual behavior and decisionmaking, and behavioral intentions about remaining abstinent. It is perhaps most important to measure actual behavior - did youth remain abstinent or delay onset of first sex, or did they not.. Programs in school settings sometimes have trouble getting permission to ask about actual behaviors, but OAPP should be encouraging collection of this information and should provide a common set of questions to use in obtaining the answers. We understand that OAPP is well advanced in developing such a core set of measures and gaining clearance to use them. We think this will be an important step forward in helping OAPP learn the most from the AFL demonstration programs.

Concepts and behaviors in addition to sexual abstinence are also important to OAPP and many of its grantees. As chapter 2 detailed, much is known about interventions that can strengthen youth assets known to be associated with delay of first sex, as well as many other positive outcomes for youth. There is also some evidence for direct effects on delay of first sex. It would be in OAPP's interest to be able to offer standardized measures for these additional concepts, including the various personal, family, and community assets and development objectives pertinent to youth development program components; abstinence from alcohol and drug abuse and other high risk behaviors; and school performance.

\section{STRENGTHENING INDIVIDUAL PROGRAM EVALUATIONS}

One of the primary purposes of conducting site visits to programs with highly developed YD components was to determine whether it would be possible to separate the effects of abstinence education from the effects of YD-oriented activities. If such separation were possible, it would then be possible to assess how much "adding YD to abstinence" augmented the effectiveness of the abstinence components. This section describes the evaluations currently under way in the seven programs, and makes suggestions for how those evaluations could be strengthened. The findings have obvious implications for overall evaluation design for AFL programs, which we discuss after presenting the findings, which are summarized in table 5.1.

\section{TyPE OF EVALUATION}

Throughout this section we will talk about "single-difference" and "double-difference" evaluation designs. A "single-difference" design involves one group of people who are assessed at two different time points (before and after receiving an intervention). Their "before" results are compared to their "after" results and the differences are examined to see whether they are big enough to claim that the intervention caused the change. This is "single-difference" because there is only one group, and only one change (before to after) that is being examined. 
When a control or comparison group is added the same testing procedures are followed, but now there are two groups for which comparisons can be made between their scores before the 


\begin{tabular}{|c|c|c|c|c|c|c|c|c|c|c|c|c|}
\hline \multicolumn{13}{|c|}{ TABLE 5.1: SUMMARY OF EVALUATION ISSUES IN SEVEN PROGRAMS VISITED } \\
\hline $\begin{array}{l}\text { Program } \\
\text { Mane }\end{array}$ & $\begin{array}{l}\text { Organization } \\
\text { Matne }\end{array}$ & Lacation & $\begin{array}{c}\text { Type of } \\
\text { Evaluation }\end{array}$ & $\begin{array}{c}\text { Comparison } \\
\text { Group? }\end{array}$ & $\begin{array}{l}\text { Control } \\
\text { Group? }\end{array}$ & $\begin{array}{l}\text { Jypes of } \\
\text { Questions }\end{array}$ & $\begin{array}{l}\text { What is } \\
\text { tested? }\end{array}$ & Assets Covered in Evaluation & $\begin{array}{l}\text { Able to } \\
\text { Measure } \\
\text { Dosage? }\end{array}$ & $\begin{array}{l}\text { Other Longterm } \\
\text { Indicators } \\
\text { Currently Tested }\end{array}$ & $\begin{array}{l}\text { Other Long-tenn } \\
\quad \text { Indicatara } \\
\text { Poosible to Tnst }\end{array}$ & $\begin{array}{l}\text { Recominendationa for } \\
\text { improved Evaluation }\end{array}$ \\
\hline $\begin{array}{l}\text { Peer Leader } \\
\text { Program (P.P1 }\end{array}$ & $\begin{array}{l}\text { Peoples Regional } \\
\text { Oppartinity } \\
\text { Program }\end{array}$ & $\begin{array}{l}\text { Patimol: } \\
\text { Mainis: } \\
\end{array}$ & $\begin{array}{c}\text { Preppost } \\
\text { Test }\end{array}$ & No. & No & $\begin{array}{c}\text { Aktitudes and } \\
\text { Gangriar }\end{array}$ & $\begin{array}{c}\text { Sexeual } \\
\text { Behasuor and } \\
\text { Piste-Taking } \\
\text { Eehavars } \\
\end{array}$ & $\begin{array}{c}\text { Youth Risk Behaviona Surviliance: } \\
\text { tems, ncluding resiliency, decision- } \\
\text { makng skills gual setiing, prosocial } \\
\text { roms, and cutura. prodf }\end{array}$ & \begin{tabular}{|} 
Yes-Hours \\
of Programi \\
Partacioarion \\
\end{tabular} & None & Hone & 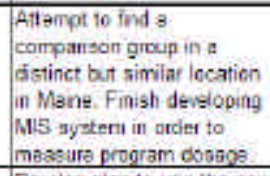 \\
\hline $\begin{array}{l}\text { The Red Deal } \\
\text { (TRO) }\end{array}$ & $\begin{array}{l}\text { Beee Hagalath } \\
\text { lenstiturese }\end{array}$ & $\begin{array}{l}\text { Erookltyn } \\
\text { Now Yoik }\end{array}$ & $\begin{array}{c}\text { Preipest } \\
\text { Tost }\end{array}$ & Yes-Matched & No & $\begin{array}{c}\text { Konawiodge } \\
\text { Attitudes } \\
\text { Some } \\
\text { Bohaviar } \\
\text { intertiens sid } \\
\text { Actial } \\
\text { Behariar } \\
\end{array}$ & $\begin{array}{c}\text { Bexual } \\
\text { Behariar } \\
\end{array}$ & 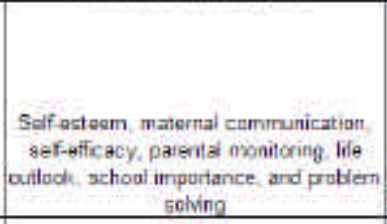 & \begin{tabular}{|} 
Yes-Heurs \\
of Pragram \\
Participation \\
\end{tabular} & Nane & \begin{tabular}{|c|} 
School \\
complation, \\
achiesemert and \\
progioss and \\
Collegs \\
atlendance and \\
porfuminase \\
\end{tabular} & 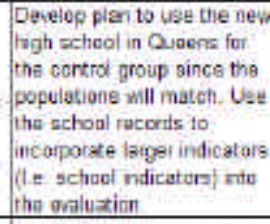 \\
\hline $\begin{array}{l}\text { Peer } \\
\text { Leaderahio } 101 \\
\end{array}$ & $\begin{array}{l}\text { The Weliness } \\
\text { Certer, Croxar } \\
\text { Chester } \\
\text { Cominumity } \\
\text { Hospingl }\end{array}$ & \begin{tabular}{l|} 
Chester; \\
Pennsybaris
\end{tabular} & $\begin{array}{c}\text { Preff }_{\text {ont }} \\
\text { Test }\end{array}$ & $\begin{array}{l}\text { Yea-Nat } \\
\text { Matched }\end{array}$ & No & $\begin{array}{c}\text { Knoudedge } \\
\text { Attitudes. } \\
\text { Behawiar } \\
\text { htertions } \\
\end{array}$ & $\begin{array}{c}\text { Sexual } \\
\text { Behanor and } \\
\text { Substance } \\
\text { UsB }\end{array}$ & 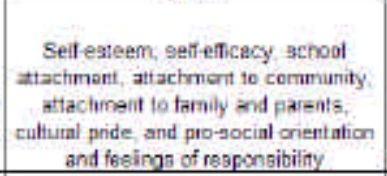 & $\begin{array}{l}\text { Yes. Heuns } \\
\text { of Pragram } \\
\text { Participation } \\
\end{array}$ & None & $\begin{array}{c}\text { School } \\
\text { completion and } \\
\text { achiaremant. }\end{array}$ & 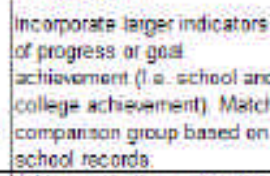 \\
\hline $\begin{array}{l}\text { Taraching } \\
\text { Responsibla } \\
\text { Actoona in Lift } \\
\text { (TRAAlL) }\end{array}$ & $\begin{array}{l}\text { Pubic Heath } \\
\text { Authorty of } \\
\text { Cabarrus }\end{array}$ & $\begin{array}{l}\text { Kannapois } \\
\text { horth } \\
\text { Caruins }\end{array}$ & $\begin{array}{c}\text { Prepost } \\
\text { Test }\end{array}$ & $Y_{25}$ & No & $\begin{array}{l}\text { Kinuuledge } \\
\text { Attitudes; } \\
\text { Behowior } \\
\text { htertiars } \\
\end{array}$ & $\begin{array}{c}\text { Bawual } \\
\begin{array}{c}\text { Banavior and } \\
\text { Substance } \\
\text { Usa }\end{array}\end{array}$ & $\begin{array}{l}\text { Gusi setuing, cormiurication, attiuded } \\
\text { tuarand toan paramtimg and zeffeficary }\end{array}$ & $\begin{array}{l}\text { Yes-Hours } \\
\text { or Program } \\
\text { Participatian }\end{array}$ & Nane: & \begin{tabular}{|c|} 
\\
Schuol \\
complation, \\
achienemeis and \\
proploss
\end{tabular} & 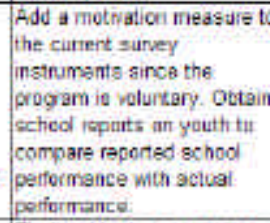 \\
\hline $\begin{array}{l}\text { The Childeri's } \\
\text { Council }\end{array}$ & $\begin{array}{l}\text { The Chideris } \\
\text { Counal }\end{array}$ & $\begin{array}{l}\text { Lancaster. } \\
\text { South } \\
\text { Caroina }\end{array}$ & $\begin{array}{c}\text { Prepest } \\
\text { Test }\end{array}$ & $\mathrm{Na}$ & No & & $\begin{array}{l}\text { Sexual } \\
\text { Batheror }\end{array}$ & 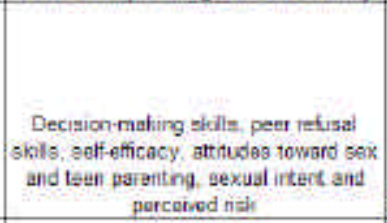 & $\begin{array}{l}\text { Yes. Hours } \\
\text { of Program } \\
\text { Participation }\end{array}$ & Nana & \begin{tabular}{|c|} 
School \\
complation. \\
achisemert and \\
progross
\end{tabular} & 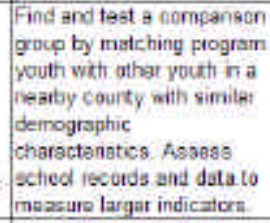 \\
\hline $\begin{array}{l}\text { Fanily Action } \\
\text { Wedel for } \\
\text { Empowoirmant } \\
\text { FASAE] }\end{array}$ & BETR Center & $\begin{array}{l}\text { Orianda } \\
\text { Flonda }\end{array}$ & $\begin{array}{l}\text { Pratphet } \\
\text { Test }\end{array}$ & No & No & 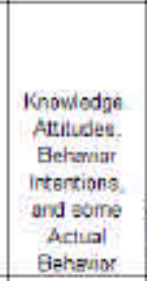 & $\begin{array}{c}\text { Sexcual } \\
\text { Bohautor and } \\
\text { Riek Taking } \\
\text { Gehasuars }\end{array}$ & 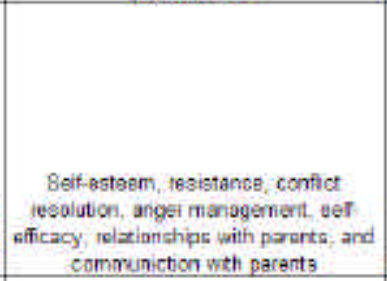 & $\begin{array}{l}\text { Yes Houre } \\
\text { of Program } \\
\text { Participation }\end{array}$ & Nane & \begin{tabular}{|c|} 
Hone \\
\end{tabular} & 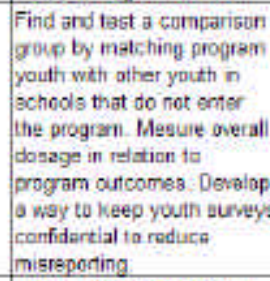 \\
\hline $\begin{array}{l}\text { Fith Wart } \\
\text { Eninchenent } \\
\text { Program } \\
\text { FWVEP }\end{array}$ & $\begin{array}{l}\text { Firtu Ward } \\
\text { Enrichmant } \\
\text { Program }\end{array}$ & $\begin{array}{l}\text { Houston, } \\
\text { Texas }\end{array}$ & $\begin{array}{c}\text { Preiphest } \\
\text { Test }\end{array}$ & $\gamma_{\text {es- } \text { - Matched }}$ & No & $\begin{array}{l}\text { Knowiedge } \\
\text { Autilidee. } \\
\text { Bahaviar. } \\
\text { htertions } \\
\end{array}$ & $\begin{array}{c}\text { Sexcual } \\
\text { Bohautor and } \\
\text { Riek Taking } \\
\text { Gehasars } \\
\end{array}$ & 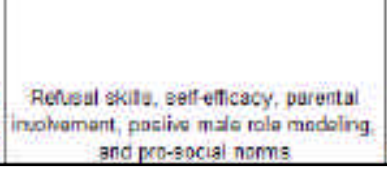 & $\begin{array}{l}\text { Yes. Houre } \\
\text { d Program } \\
\text { Particigation }\end{array}$ & $\begin{array}{c}\text { Sehool } \\
\text { campletion and } \\
\text { pethrmance. } \\
\text { Colege } \\
\text { athendarce and } \\
\text { performance }\end{array}$ & $\begin{array}{l}\text { Schoel } \\
\text { completion and } \\
\text { perforaance, } \\
\text { College } \\
\text { attandance and } \\
\text { pertomance }\end{array}$ & 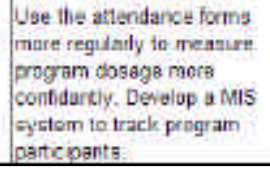 \\
\hline
\end{tabular}


intervention and their scores after the intervention-hence "double-difference." Ideally, the scores of the intervention group change and the scores of the control or comparison group do not, lending more weight to the idea that the intervention caused the change rather than the mere passage of time. If the two groups are created by randomly assigning people to one or the other, the nonintervention group may properly be termed a "control" group. With a true control group, the randomized assignment process is assumed to control for many things in addition to time that might alter the interpretation that the intervention caused the observed changes - things such as differences in the characteristics or propensities of the group members to do the things that the intervention is trying to alter. With random assignment an evaluator may feel confident that as much as possible has been done to make the two groups as equivalent as possible at the start of the intervention. This most scientifically rigorous evaluation design allows more confidence that any differences observed are true differences attributable to the intervention. Control or comparison groups are particularly important for interventions aimed at preventing a negative outcome from occurring, because they are the only way to show that without the intervention there would have been more of the negative outcome. ${ }^{11}$

Each of the seven programs we visited has designed and implemented a fairly straightforward pre-/post-test evaluation process. None has a true control group, but some have comparison groups. Thus we have both single-difference and double-difference designs among the seven programs.

Generally, the adolescents who participate in the youth program take a pre-test during the first two weeks of the program and the results of these pre-tests are generally entered into a database by the program staff or the evaluator. During the last several weeks of the program, (whether it be eight weeks after the initial session or a full school year later), the adolescents answer a posttest that covers much of the same information as the pre-test. The responses of these two tests are compared to see the "effectiveness" of the program in imparting new knowledge or in changing youth attitudes, behavior intentions, or actual behaviors.

\section{CONTROL AND COMPARISON GROUPS}

Currently, none of the seven programs use a control group of adolescents to track the success of the program in influencing youth over time. Four programs (Peer Leadership 101, Fifth Ward Enrichment Program, The Real Deal, and Teaching Responsible Actions in Life) do use comparison groups, giving them "double-difference" designs that have some greater claim to scientific rigor than the single-difference designs in the remaining three programs visited. The adolescents in the comparison groups do not receive the youth development and abstinence education programming but do participate in the pre-/posttests of the evaluation. Comparison groups are obtained through connections to the schools in which parts of the programs occur. Partner schools and school districts allow the AFL programs to conduct their surveys in whole

\footnotetext{
${ }^{11}$ Some may remember the old joke about the man who covered a street with newspapers "to keep the elephants away." When onlookers protested that there were no elephants, his response was "see, it's working." But most people would recognize that the street would not be full of elephants even it if were not covered with newspapers. A prevention intervention without a control-group evaluation design is in the same position as the newspaperspreading man-it cannot show that the intervention was needed to bring about the result.
} 
classrooms not receiving the interventions, in schools not receiving the interventions, or by calling youth not receiving the interventions to cafeterias or auditoriums to administer the preand postintervention surveys.

Notable differences exist among the four programs using comparison groups in the ways these groups are constructed. Two of the evaluation designs use matched comparison groups, for which students are selected for the comparison group based on common characteristics they share with program participants. The two evaluations that do not use matched comparison groups may be comparing apples to oranges because the two groups of adolescents may have totally different demographic characteristics, knowledge about and attitudes towards sexual behaviors, and distinct life experiences.

Two programs are thinking about or doing variations on comparison groups that might be possible for the evaluators in other AFL-funded programs. For its most recent program year (results not yet known), one program developed a variation on the technique of calling comparison group students to the auditorium. The evaluator worked with participating schools to match students not in the program to those who do participate, using a number of individual characteristics known at the beginning of the program year. This matching procedure is a good way to improve the similarities between intervention and comparison groups when true random assignment is not possible. Another program randomized students into treatment and nontreatment groups each year, but was also committed to having each student participate in the program for at least one high school year. The consequence was that everyone in the school got at least some level of the intervention and no true control or comparison group existed. However, analyzing the results by "dosage,"- the number of months of participation, which ranges from 12 to 48 - was possible and will probably be done for the next evaluation report.

\section{TYPES OF QUESTIONS AND TOPICS TESTED}

We received detailed evaluation information from six programs visited about the tests they use to assess knowledge of and attitudes toward sexual behaviors (and some other risk-taking behaviors). Five programs test behavior intentions related to sexual behavior and three programs test actual behavior-including if the participants have engaged in sexual intercourse (the People's Regional Opportunity Program in Portland, Maine does not test behavior intentions but does test actual behavior; the evaluation instrument asks if the child has engaged in sexual behaviors but does not ask if the child intends to do so in the future.).

In addition to gathering data with respect to sexual behavior, five of the seven programs visited inquire about other risk-taking behaviors such as substance use. These programs are trying to promote safe and prosperous futures for their participants, which means healthy decisionmaking and positive behavior choices with respect to a broad array of risky behaviors and asset development. Sexual abstinence is an important, but not the only, focus for abstinence. At least one program, FWEP in Houston, Texas, goes so far as to work with youth to abstain from bad language, "gangsta" dress, and so on, reasoning with youth that they should be looking and behaving as they want to look and behave as adults. 


\section{OTHER LONG-TERM INDICATORS CURRENTLY B EING TESTED}

Only Fifth Ward Enrichment Program in Houston, Texas tests indicators related to school completion and performance and college attendance and performance, although several other programs visited could do so with relative ease. FWEP believes that an adolescent's performance in school can be related to the assets he has built in other aspects of his life. FWEP also views attending college as an indicator of positive youth development and sound decisionmaking skills. It did a stand-alone assessment one year, when it had the money for the study, to see whether participation improved school attendance, performance, and completion. (It did.) The program also makes a concerted effort to maintain contact with participants for years after the students complete the program and leave for higher education. Many FWEP alumni return to the program to visit and even participate in advisory roles. Alumni often participate in career activities program components to show what a FWEP participant can achieve.

The other six programs do not currently examine their participants' school attendance and performance, although most affirm that improving school performance is part of their larger program purpose. Four programs (Peer Leadership 101, The Real Deal, Teaching Responsible Actions in Life, and The Children's Council) have the infrastructure in place to observe and track their participants' academic experiences in middle and high school. Several of these programs work closely with the school districts on a consistent basis and could use those connections to receive students' grades and reports as well as attendance records. The Real Deal program in New York could even track students as they enter college because of their close academic and personal relationships with students. Further, as the only program visited for which AFL funding is relatively recent (the others have had previous AFL grants), The Real Deal could go back to school records three years ago and assess whether the changes in confidence, academic success, and life trajectories that staff perceive to be so changed really can be documented by comparing to the previous high school cohort of girls.

\section{ANALYSES REPORTED AND OTHER OPPORTUNITIES FOR UNDERSTANDING PROGRAM EFFECTS}

Evaluation reports for these seven programs present very simple tabulations. They usually show pretest scores and posttest scores as percentages, revealing "single-differences" that have occurred. If they have information for a comparison as well as an intervention group, they show these data also, and note the variables on which the intervention group changed, or changed more, than the comparison group (a double-difference design).

None of the programs visited use any information about levels of program participation (how many hours, weeks, or months each student participated) to help explain results, although such analyses would add greatly to the value of the single-difference evaluations and also help understand any differences (or lack of differences) found in the double-difference designs. All the programs we visited collect dosage information (they keep attendance at each event or class), although some do it more consistently and completely than others. Most of the programs also automate this information, and others are working toward having the information automated. We strongly suggest that dosage information be routinely reported to OAPP as part of year-end reports, and that it be used in evaluation analyses to help understand whether more participation leads to better outcomes. This would also help differentiate among programs in which youth 
could not participate for lengthy periods (because the program length is short) from those in which youth may participate for years, and reveal whether the longer programs have stronger effects, as they do in much of the youth development literature.

None of the programs visited conduct any type of long-term follow-up to see how long the effects of intervention last. Even those programs in which youth can participate for several years (5 of the 7 programs visited) do not compare the same children from year to year. That is, they conduct a pretest at the beginning of each program year and a posttest at the end of each program year, but do not compare posttests from year 1 to posttests from year 2, to see if effects have held or strengthened. Such analyses would be relatively simple to do, since the programs already have the relevant data, but they do not do them. They would also be most informative, to OAPP and also to the programs themselves. Any program that does not see year-to-year progress, especially with respect to behavioral intentions and actual behavior, should examine its interventions to assess what could make it more effective.

\section{PROGRAM-SECIFIC RECOMMENDATIONS FOR IMPROVING EVALUATIONS}

Evaluation designs for each program visited might benefit from some small changes to make them more informative and therefore valuable. We offer recommendations to each program to improve its evaluation, all of which were discussed with program staff and evaluators during site visits. ${ }^{12}$ Other OAPP programs could also implement some of these evaluation improvements.

Peer Leader Program (PLP), Maine: PLP should finish developing its management information system (MIS) so it can increase the consistency with which it measures program dosage. PLP is currently working on this task and we think it will help them better determine program effectiveness in helping participants achieve program goals. We also suggest, although we realize it might be difficult for PLP to accomplish given the unique nature of its youth (refugees and immigrants), that the evaluator attempt to find a comparison group in a distinct but similar location in Maine. While we do not know the feasibility of this, we recommend PLP find a similar refugee/immigrant population not receiving PLP programming so it can compare the two groups of youth using pre-/posttests.

The Real Deal, New York: The Real Deal program has the potential for an excellent evaluation design since the program is run by the school the participants attend. The Real Deal program staff and evaluator could develop a plan to use the new Be'er Hagolah High School in Queens (beginning with the 2005-2006 school year) to create a comparison group of girls who do not receive the youth development and abstinence education programming offered in the Brooklyn school. This seems possible given that The Real Deal will not be implemented in the Queens school for some time so the female students will not be deprived of this component. This is an excellent opportunity for Be'er Hagolah to attempt a true control group evaluation design. In addition, we recommend that (1) The Real Deal use school records to incorporate school

\footnotetext{
${ }^{12}$ The program-specific recommendations are offered as feasible next steps to build on the current evaluation design of each program. As some programs currently have more sophisticated designs than others, the "next steps" for the programs with the less sophisticated designs may already be a part of evaluation designs in other programs. The "next steps" for the programs with more sophisticated current designs would also be appropriate for the remaining programs, but are not likely to be possible until the programs take their own "next steps."
} 
indicators into the evaluation and relate them to the number of months or years that girls have participated; (2) as it has ready access to the information, the program should also include posthigh school educational successes, as well as participation in prosocial activities in college; (3) the evaluator should go back and use girls' academic performance, college attendance, and community participation for the high school cohort that graduated just before The Real Deal started, and compare to the present. This third addition to the evaluation design will help the program answer its own question of whether The Real Deal has made a significant difference over and above the already nurturing and supportive orientation of the whole school.

Peer Leadership 101, Pennsylvania: While this program has already changed, in its most recent evaluation year, to use a matched comparison group, the program might be able to find a more useful and realistic method than its present practice for matching its students and the comparison group. We suggest trying to match the students based on school records since the program has strong ties to the school system and local teachers. In addition, we recommend that Peer Leadership 101 incorporate indicators of progress in several domains of goal achievement into its evaluation design. Some examples might be school performance and college attendance and performance. We also recommend that it compare student results from year to year to track persistence or dilution of effects.

Teaching Responsible Actions in Life (TRAIL), North Carolina: Currently, participants in this program report their school performance (grades and reports) on the evaluation instruments. We suggest TRAIL obtain school reports on youth to compare the reported school performance with actual performance. Also, we recommend that TRAIL add a motivation measure to the current interview instrument to determine the youth's reasons for joining the program, since participation in TRAIL is voluntary. Lastly, TRAIL should attempt to measure school completion, achievement, and progress while the students are still in secondary school since the program has strong ties with the school system.

The Children's Council, South Carolina: The Children's Council does not currently employ a comparison group evaluation design, and rectifying this gap should be its main focus for improving its evaluation. The program should find and test a comparison group by matching program youth with other youth in a nearby county with similar demographic characteristics. In addition, the evaluator should access school records to measure the broader indicators of school completion, achievement, and progress that the program also espouses. If possible, the evaluation instruments should incorporate questions to measure youth attitudes, knowledge, and intentions regarding other risk-taking behaviors in addition to sexual behavior.

Family Action Model for Empowerment (FAME), Florida: The main focus of FAME's evaluation team should be to develop, find, and test a comparison group. The program could match program youth with other youth in neighboring schools that do not enter the program (since many students in the geographic area do not participate). The difficulty in this situation might be obtaining school records since FAME does not consistently work with the same schools year after year. In addition, we suggest that FAME measure overall program dosage in relation to program outcomes and goals. Currently, FAME collects information about how much programming each student receives, but does not analyze it in relation to his/her outcomes. Finally, we suggest that FAME develop a way to keep youth surveys confidential in order to 
reduce misreporting. FAME might consider using sealed envelopes and not checking the surveys for complete answers, especially in front of the students.

Fifth Ward Enrichment Program (FWEP), Texas: FWEP already incorporates indicators of goal achievement in its evaluation design and uses a matched comparison group. While these two aspects of its evaluation are strong, we would suggest placing some importance on incorporating program dosage as an explanatory variable in analysis of results. By using the attendance forms more regularly, the evaluator could measure program dosage more confidently to see if a higher program dosage means more positive outcomes for its participants. In addition, we would like to see FWEP develop a strong MIS to track program participants based on the attendance forms the program managers already use. Assessing school performance and year-toyear tracking would also be possible and fruitful, if adequate evaluation resources were available.

\section{IMPLICATIONS FOR IMPROVING OTHER INDIVIDUAL AFL GRANTEE EVALUATIONS}

The evaluation designs pursued by the seven programs we visited tend to be among the stronger evaluations being done by AFL grantees, as a relatively strong evaluation component was one of the criteria for selecting the sites to visit. Even these evaluations could be improved, as just noted. We can generalize from aspects of the designs as currently being conducted and suggested improvements to some recommendations to OAPP to strengthen the evaluation information it receives from individual program evaluations (as described in chapter 3).

\section{Encourage enhancements to grantee evaluations.}

While some of the evaluations of the AFL grantees were quite strong, two issues arose with a number of evaluations. The first was the lack of a clear logic model. While most evaluations were clear about how they measured program objectives, what they did not discuss was the theoretical link between the intervention components and the stated objectives. Articulating these linkages better and designing evaluations to test them will assist the grantees and OAPP staff in understanding why certain program components are effective or not.

Another issue with the evaluation was the relative small number of programs using comparison groups in their design. Fewer than half of the 45 grantees whose programs were reviewed in chapter 3 used a comparison group design — a ratio that only improved slightly among the programs site visited ( 4 of 7 programs, or 57 percent, had comparison groups). The importance of having a comparison group was discussed above.

AFL grantees should also be encouraged to measure outcomes beyond sexual behavior and to use OAPP's standardized measures to do so (see recommendation 4, above).

It is also important to note that these recommendations may be difficult to achieve given AFL grantees' limited resources for program evaluation. OAPP may want to enhance the evaluation resources for programs that commit to conducting more sophisticated designs, and that offer interventions using evidence-based practices known to reduce risky sexual and other behaviors and to achieve youth development objectives. 


\section{Provide technical assistance to grantees wishing to enhance the positive youth development strategies they employ and/or the strength of their evaluations.}

The AFL grantees whose year-end reports we reviewed (see chapter 3) varied significantly in the extent to which they emphasized positive youth development strategies and how these strategies were evaluated. The programs site visited, which we selected for their highly developed YD approaches, could potentially be used as model programs from which OAPP could draw best practices. Their evaluation approaches, coupled with the recommendations offered above for strengthening individual program evaluations, could also be used as examples of useful evaluation strategies. OAPP staff could then provide technical assistance and lessons learned to those grantees wishing to enhance their positive youth development strategies and their evaluations.

\section{STRENGTHENING OAPP'S ABILITY TO ASSESS EFFECTIVENESS ACROSS GRANTEES}

This section presents a number of options for OAPP to pursue in its efforts to improve knowledge of the effectiveness of its abstinence education programs and the role that youth development strategies may play in augmenting their impact. Each option could be pursued separately, but the strongest approach would be to do the first, then use all the rest in a systematic cross-program evaluation conducted in addition to individual grantee efforts. The options are: a meta-analysis on existing program evaluations, cross-program evaluation design, follow-up data collection and year-to-year analyses to determine the persistence of effects, incorporating client difficulty information into analyses, and assessment of cost-effectiveness against desirable program outcomes, not just participants served.

\section{Perform a Meta-Analysis of Existing Evaluations}

This project reviewed available evaluation reports to identify different designs, outcome measures, and other evaluation elements. But we did not have the resources to conduct a metaanalysis of the actual outcomes described in these reports. A meta-analysis of findings from existing AFL grantee evaluations will be difficult but not impossible to do, as both programs and evaluations vary so much and OAPP files do not always contain needed information. One would need two types of information: (1) program descriptions and (2) evaluation outcomes.

For program descriptions, one could use the program characteristics that our review identified (i.e., duration, frequency, presence of YD components, all as indicated by year-end or evaluation reports) as a starting point. As only 60 percent of program reports provided adequate information on these characteristics, a first step would be to obtain them for all programs, either by requiring full information in the next program reports or by calling programs for the missing information.

Extracting evaluation outcomes will be somewhat more challenging. The first step, as with program descriptions, would be to obtain all available outcome information, aga in calling programs to complete files where this information is currently missing. The second step would be to array all the outcomes measured against all the measures used and then look for 
commonalities across programs. Most programs will have assessed knowledge of sexual behaviors before and after program participation. Almost as many programs will have assessed attitudes toward sexual behaviors. They will of course have used different measures and different questions, and may have reported results in different ways. Beyond knowledge and attitudes with respect to sexual behavior, commonalities will drop considerably, but as behavioral intentions and actual behaviors with respect to sexual and other risk taking and positive youth outcomes are what OAPP is really interested in, it will still be most important to examine what AFL grantees are actually doing and reporting on these variables.

The meta-analyst will have to identify the variables for which sufficient data exist to perform cross-program analyses, and for which measures are sufficiently similar to make such analyses defensible. The cross-program results will give OAPP a useful handle on some of its most commonly measured outcomes, and also identify the major gaps in information that a good cross-site evaluation should seek to fill.

\section{Conduct a Major Cross-Program Evaluation}

Given that the AFL grantees with the most YD content have program approaches that do not lend themselves to separating the effects of abstinence education and YD strategies, we sought alternative evaluation designs OAPP might pursue with its existing and potential future grantees. We think the best alternative is to compare AFL grantee programs to each other, first stratifying the programs by their intensity and YD content/approach. Grouping by intensity alone might also be an option. However, this would not assure OAPP that it also had the amount of variation in YD content and approach needed to examine its primary question of whether incorporating YD enhances the effects of abstinence education. To be sure of sufficient YD variation, groups should be formed with an eye to both intensity and YD content/approach. We suggest several possible categories, but the final ones will have to depend on the actual configurations of grantee programs.

5. No YD, low intensity_Grantees with very little or no YD component, whose interventions are classroom only and of relatively low intensity (i.e., one semester or less);

6. No YD, medium intensity-Grantees with very little or no YD component, but whose interventions include some component that goes beyond school classrooms and also lasts at least a whole school year;

7. Some YD, medium intensity-Grantees that combine YD and abstinence, but are short, or don't meet very often, or are classroom only, or some combination of these traits;

8. Lots of YD and high intensity-Grantees such as many of those we visited, with major YD components and high intensity.

Select some programs from each group and develop a consistent evaluation design across sites. One might think about omitting group 1, as most evidence suggests these short school-based abstinence-only programs affect, at most, knowledge and attitudes, and that their effects decay over time (see chapter 2). Alternatively one could combine groups 1 and 2 and use some measures of their intensity and classroom/other approaches as a way to characterize their interventions and compare them to programs in groups 3 and 4 . Whatever the groupings, one 
would want to set up a fair test. One would not want to use the group 1 programs just as a foil against which to show the (expected) greater effects of the more intensive programs. On the other hand, if group 1 programs really are significantly less effective than programs in other groups, one would want to take that information into account in deciding what to fund.

The design developed should follow many of the suggestions given earlier in this chapter for strengthening individual program evaluations, including reasonable comparison groups, measures of dosage by the variety of ways that content is conveyed and by the specific types of content offered, and inclusion of a broader array of outcome variables (e.g., school performance, abstinence from many types of risky behaviors, relationships with parents and teachers).

The evaluation should take particular pains to measure both the content and mechanisms that programs use to deliver abstinence messages, and do the same for activities promoting YD objectives. Without really good ways to describe what these programs actually do with youth, one would not gain the most knowledge from a cross-site evaluation such as we are describing.

Analyses should include multivariate methods to help sort out the effects of programs with a complex array of components on a range of dependent variables. Finally, as new grants are awarded, OAPP might consider how to help them incorporate data collection and evaluation that will provide good data for decisionmaking.

\section{The Cross-Program Evaluation Should Include Assessments of How Dosage and Client Difficulty Affect Results}

It will be very important to include in any cross-program evaluation design the collection and analysis of variables describing the youth participants of AFL programs. We have already discussed at some length the importance of including measures of client participation levels ("dosage") in analyses, to see if attending more sessions, over more weeks, months, or years, affects the level and persistence of desirable outcomes. All available evidence (summarized in chapter 2) indicates that longer, more intensive, and more comprehensive programs have more long-lasting effects. OAPP would want to be able to compare its results to those of previous studies to show how its programs fit into the full array of youth-serving programs.

"Client difficulty" is another aspect of youth participants that it is most important to measure and include in analyses. Programs that take only very "easy" youth-those from relatively problemfree neighborhoods and with relatively supportive families-are likely to show better results with less effort than programs that take youth with more problems, or coming from more troubled neighborhoods that expose youth to more opportunities to engage in risky behavior. A crossprogram evaluation should collect information about client, family, and neighborhood characteristics that could affect the ease or difficulty of obtaining desirable program outcomes. Some subset of these variables will be important to include in impact analyses.

\section{Include Follow-Up Assessments of Impact}


The strongest programs should impact not only the short-term sexual behaviors and intentions of adolescents, but also should send them on a trajectory that minimizes risk-taking behavior in the long term. Given the need for programs that affect children for the long term and potential for "fade-out" effects, OAPP should encourage evaluators to conduct follow- up studies of program participants.

- For the least intense programs (e.g., one semester), these follow-ups should occur at least one full semester after participation ends, and one full year would be better. If the effects cannot endure that long, perhaps the programs are not worth funding.

- For programs in which youth participate for more than one year, analyses should be conducted to see whether effects persist and intensify from year to year, as already suggested earlier in this chapter.

- For the recommended cross-program evaluation, both longer-term follow-up and year-toyear analyses should be conducted.

\section{Conduct Cost-Effectiveness Analyses Against Desirable Program Outcomes}

More and more, government-funded programs are being asked to justify their costs by showing what their grantees are producing for the funds being invested in them. This is a very reasonable expectation, but too often it is demanded without thinking of what will provide the most meaningful indication of "value for money." To our minds, the only appropriate costeffectiveness indicator is "cost per desired outcome achieved." All too often, however, the only cost measures available, or calculated, are "cost per participant."

The problem with "cost per participant" measures is that a program can be very cheap per participant, but accomplish nothing. In that case all the money invested in the program is wasted from the perspective of achieving program goals. The goals, after all, are not just to serve people, but to change them, to move them in the direction the program sponsors want them to go.

It is a truism in evaluation research and cost analyses that investments made in the very hardestto-serve clients are the most cost-effective, even though they are also the most expensive per participant. This is so because the very hardest-to-serve clients would almost never achieve the same desirable outcomes without the program, whereas relatively large proportions of easier-toserve clients would do so even without program intervention. From a cost-effectiveness point of view, the best investments are those made in people who would not have achieved desirable outcomes without the program.

The need to defend program expenditures by showing that the investment is producing good outcomes means that OAPP should build a "cost per desirable outcome" cost-effectiveness analysis into the cross-program evaluation that is the major recommendation of this report. 


\section{REFERENCES}

Allen, J. P., G. Kuperminc, S. Philliber, \& K. Herre. 1994. "Programmatic Prevention of Adolescent Problem Behaviors: The Role of Autonomy, Relatedness, and Volunteer Service in the Teen Outreach Program." American Journal of Community Psychology 22: 617-38.

Allen, J. P., S. Philliber, S. Herrling, \& G. P. Kuperminc. 1997. "Preventing Adolescent Pregnancy and Academic Failure: Experimental Evaluation of a Developmentally Based Approach." Child Development 64(4): 729-42.

Andrews, D. W., L. H. Soberman, \& T. J. Dishion. 1995. "The Adolescent Transitions Program for High-Risk Teens and Their Parents: Toward a School-Based Intervention." Education and Treatment of Children 18: 478-98.

Bearman, P., \& H. Brückner. 1999. "Peer Effects on Adolescent Sexual Debut and Pregnancy: An Analysis of a National Survey of Adolescent Girls." In Peer Potential: Making the Most of How Teens Influence Each Other. Washington, DC: National Campaign to Prevent Adolescent Pregnancy.

- 2001. "Promising the Future: Virginity Pledges and First Intercourse." American Journal of Sociology 106: 859-912.

Billy, J. O. G., K. L. Brewster, \& W. R. Grady. 1994. "Contextual Effects on the Sexual Behavior of Adolescent Women." Journal of Marriage and the Family 56: 387-404.

Blum, R. W., T. Beuhring, \& P. M. Rinehart. 2000. Protecting Teens: Beyond Race, Income and Family Structure. Minneapolis, MN: Center for Adolescent Health, University of Minnesota.

Blum, R. W., \& P. M. Rinehart. 2002. Mothers' Influence on Teen Sex: Connections that Promote Postponing Sexual Intercourse. Minneapolis, MN: Center for Adolescent Health and Development, University of Minnesota.

Brewster, K. L., J. O. G. Billy, \& W. R. Grady. 1993. "Social Context and Adolescent Behavior: The Impact of Community on the Transition to Sexual Activity." Social Forces 71: 713-740.

Brown, B. B., \& W. Theobald. 1999. "Peers Matter: A Research Synthesis of Peer Influences on Adolescent Pregnancy." Washington DC: National Campaign to Prevent Adolescent Pregnancy.

Cardenas, J. A., M. R. Montecel, J. D. Supik, \& R. J. Harris. 1992. "The Coca-Cola Valued Youth Program: Dropout Prevention Strategies for At-Risk Students." Texas Researcher 3:11130 .

Catalano, R. F., M. L. Berglund, J. A. M. Ryan, H. S. Lonczak, \& J. D. Hawkins. 2002. "Positive Youth Development in the United States: Research Findings on Evaluations of Positive Youth 
Development Programs." Prevention and Treatment 5, article 15.

http://journals.apa.org/prevention/volume5/pre0050015a.html, accessed August 29, 2005, posted

June 24, 2002.

—. 2004. "Positive Youth Development in the United States: Research Findings on

Evaluations of Positive Youth Development Programs." Annals of the American Academy 591:

98-124.

Development Studies Center. 2002. "Summary of Evaluation Findings on the Child Development Project." http://www.devstu.org/cdp/pdfs/cdp_eval_summary.pdf, accessed January 15, 2005.

DiCenso, A., G. Guyatt, A. Willan, \& L. Griffith. 2002. "Interventions to Reduce Unintended Pregnancies Among Adolescents: A Systematic Review of Randomized Controlled Trials." BMJ 324: $1426-30$.

Dorius, G. \& B. Barber. 1998. Parental support and control and the onset of sexual intercourse. Unpublished manuscript, Brigham Young University, Provo, Utah [cited in Miller, 2002]

DuBois, D. L., B. E. Hollaway, J. C. Valentine, \& H. Cooper. 2002. "Effectiveness of Mentoring Programs for Youth: A Meta-Analytic Review." American Journal of Community Psychology 30: 157-97.

Hahn, A., T. Leavitt, \& P. Aaron. 1994. Evaluation of the Quantum Opportunities Program: Did the Program Work? Waltham, MA: Brandeis University, Heller Graduate School.

Halpern, C. T., K. Joyner, J. R. Udry, \& C. Suchindran. 2000. "Smart Teens Don't Have Sex (Or Kiss Much Either)." Journal of Adolescent Health 26: 213-25.

Hawkins, J. D., J. Guo, K. G. Hill, S. Battin-Pearson, \& R. D. Abbott. 2001. "Long-Term Effects of the Seattle Social Development Intervention on School Bonding Trajectories." Applied Developmental Science 5: 225-36.

Hofferth, S. L. 1987. "Factors Affecting the Initiation of Sexual Intercourse." In Risking the Future: Adolescent Sexuality, Pregnancy and Childbearing, edited by S. Hofferth \& C. D. Hayes (7-35). Washington DC: National Academy Press.

Johnson, K., T. Strader, M. Berbaum, D. Bryant, G. Bucholtz, D. Collins, \& T. Noe. 1996. "Reducing Alcohol and Other Drug Use by Strengthening Community, Family, and Youth Resiliency: An Evaluation of the Creating Lasting Connections Program." Journal of Adolescent Research 11: 36-67.

Kirby, D. 2001. Emerging Answers: Research Findings on Programs to Reduce Adolescent Pregnancy. Washington, DC: National Campaign to Prevent Adolescent Pregnancy. 
Maddox, S. J., \& R. J. Prinz. 2003. "School Bonding in Children and Adolescents:

Conceptualization, Assessment, and Associated Variables." Clinical Child and Family

Psychology Review 6: 31-49.

Manlove, J., K. Franzetta, K. McKinney, A. Romano-Papillo, \& E. Terry-Humen. 2004. A Good Time: After-School Programs to Reduce Adolescent Pregnancy. Washington, DC: National Campaign to Prevent Adolescent Pregnancy.

Manlove, J., A. Romano-Papillo, \& E. Ikramullah. 2004. Not Yet: Programs to Delay First Sex Among Teens. Washington, DC: National Campaign to Prevent Adolescent Pregnancy.

McBride, D., \& A. Gienapp. 2000. "Using Randomized Designs to Evaluate Client-Centered Programs to Prevent Adolescent Pregnancy." Family Planning Perspectives 32: 227-35.

Miller, B. C. 1998. Families Matter: A Research Synthesis of Family Influences on Adolescent Pregnancy. Washington, DC: National Campaign to Prevent Adolescent Pregnancy.

- 2002. "Family Influences on Adolescent Sexual and Contraceptive Behavior." Journal of Sex Research 39: 22-26.

Miller, B. C., M. C. Norton, T. Curtis, E. J. Hill, P. Schvaneveldt, \& M. H. Young. 1997. "The Timing of Sexual Intercourse among Adolescents: Family, Peer, and Other Influences." Youth and Society 29: 54-83.

National Research Council and Institute of Medicine. 2002. Community Programs to Promote Youth Development: Executive Summary. Washington DC:National Academy Press.

NRC/IOM. See National Research Council and Institute of Medicine.

O’Donnell, L. O., A. Stueve, A. San Doval, R. Duran, D. Haber, R. Atnafou, N. Johnson, U. Grant, H. Murray, G. Juhn, J. Tang, \& P. Piessens. 1999. "The Effectiveness of the Reach for Health Community Youth Service Learning Program in Reducing Early and Unprotected Sex Among Urban Middle School Students." American Journal of Public Health 89: 176-81.

Orr, D., M. Wilbrandt, C. Brack, C. Rauch, \& G. Ingersoil. 1989. "Reported Sexual Behaviors and Self-Esteem Among Young Adolescents." American Journal of Disabled Children 143: 8690 .

Pedro-Carroll, J. L., \& E. L. Cowen. 1985. "The Children of Divorce Intervention Program: An Investigation of the Efficacy of a School-Based Prevention Program." Journal of Consulting and Clinical Psychology 53: 603-11.

Philliber, S., J. W. Kaye, S. Herrling, \& E. West. 2002. "Preventing Pregnancy and Improving Health Care Access among Teenagers: An Evaluation of the Children's Aid Society-Carrera Program." Perspectives on Sexual and Reproductive Health 34: 244-51. 
Resnick, M. D., P. S. Bearman, R. W. Blum, K. E. Bauman, K. M. Harris, J. Jones, J. Tabor, T. Beuhring, R. E. Sieving, M. Shew, M. Ireland, L. H. Bearinger, \& J. R. Udry, 1997. "Protecting Adolescents from Harm: Findings from the National Longitudinal Study on Adolescent Health." Journal of the American Medical Association 278: 823-32.

Robinson, R. B., \& D. Frank. 1994. "The Relation between Self-Esteem, Sexual Activity, and Pregnancy." Adolescence 29: 27-35.

Salazar, L. f., R. A. Crosby, R. J. DiClemente, G. M. Wingood, C. M. Lescano, \& L. K. Brown. 2005. Self-esteem and theoretical mediators of safer sex among African-American female adolescents: Implications for sexual risk reduction interventions. Health Education and Behavior, 32(3): 413-427.

Schinke, S. P., G. J. Botvin, J. E. Trimble, M. A. Orlandi, L. D. Gilchrist, \& V. S. Locklear. 1988. "Preventing Substance Abuse Among American-Indian Adolescents: A Bicultural Competence Skills Approach.” Journal of Counseling Psychology 35: 87-90.

Sieving, R. E., C. S. McNeely, \& R. Blum. 2000. "Maternal Expectations, Mother-Child Connectedness, and Adolescent Sexual Debut." Archive of Pediatric Adolescent Medicine 154: 809-16.

Slavin, R. E., N. A. Madden, L. J. Dolan, B. A. Wasik, S. Ross, L. Smith, \& M. Dianda. 1996. "Success for All: A Summary of Research." Journal of Education for Students Placed At Risk 1: 41-76.

Sonenstein, F. L., S. Punja, and C. Scarcella. 2004. "Future Directions for Family Planning Research: A Framework for Title X Family Planning Service Delivery Improvement Research." Washington, DC: The Urban Institute.

Spencer, J. M., G. D. Zimet, M. C. Aalsma, \& D. P. Orr. 2002. "Self-Esteem as a Predictor of Initiation of Coitus in Early Adolescents.” Pediatrics 109: 581-84.

Tierney, J. P., \& J. B. Grossman. 2000. Making a Difference: An Impact Study of Big Brothers Big Sisters. Philadelphia, PA: Public/Private Ventures.

Walker, G., \& F. Vilella-Velez. 1992. The Summer Training and Education Program (STEP) from Pilot through Replication and Postprogram Impacts. Philadelphia, PA: Public/Private Ventures.

Zabin, L. S., M. B. Hirsch, E. A. Smith, R. Streett, \& J. B. Hardy. 1986. Evaluation of a pregnancy prevention program for urban teenagers. Family Planning Perspectives, 18(3): 119126. 


\section{APPENDIX A, PART 1: YOUTH DEVELOPMENT IMPACTS ON TEENS' SEXUAL BEHAVIOR}

Citation

\begin{tabular}{l}
\hline Joseph Allen, Gabe \\
Kuperminc, Susan \\
Philliber, \& Kathy Herre. \\
(1994). \\
"Programmatic Prevention \\
of Adolescent Problem \\
Behaviors: The Role of \\
Autonomy, Relatedness, \\
and Volunteer Service in \\
the Teen Outreach \\
Program."
\end{tabular}

Purpose of Study

Replicate nationally the teen outreach

program to prevent school failure,

suspension, and teenage pregnancy. The

program has been documented to be

effective in reducing adolescent problem

behaviors (based on seven years of data

involving more than 6,000 treatment and comparison students in more than 130

sites nationwide). The program is a

volunteer service progr am combined

with a classroom-based curriculum to ensure discussions on various youth

topics.

\section{Research/Evaluation Design}

Site-level data from 66 sites and more

than 2,000 treatment and comparison

students were included nationwide

from 1987 through 1992. Students

entered the teen outreach program

through a health class, elective, or

after-school program. Random

assignment was not used because it

limits the examination of

intraprogram differences. A pre- and

posttest were conducted on the

treatment and well-matched

comparison groups. Comparison

groups were selected in one of the

following ways: (1) treatment students

nominated other students who had

similar characteristics; (2) school

personnel suggested which students

should be in the comparison groups;

or (3) if the program was

overenrolled, other students were

assigned to the comparison group.
Community and Setting

The 66 sites nationwide that voluntarily participated in the study did not differ in race, gender, parents' educational background, program entry/exit level, or level of problem behavior. The average age of participants was 15.5 years, and students ranged in age from 11 to 19 and were in 7 th to 12 th grade. The majority of the participants were black and white, and almost threequarters were female.

\section{Summary of Findings}

Overall, students significantly decreased their levels of problem behavior from program entry to exit (relative to the comparison groups). They reported a lower likelihood of getting pregnant (girls) or causing pregnancy (boys), failing any courses during the previous school year and being suspended during the previous year. These findings are robust when demographic characteristics are controlled. The program was successful in preventing problem behaviors when strongly linked to autonomy promoting program

characteristics, such as independent volunteer work. Evidence was strongest for middle school students.

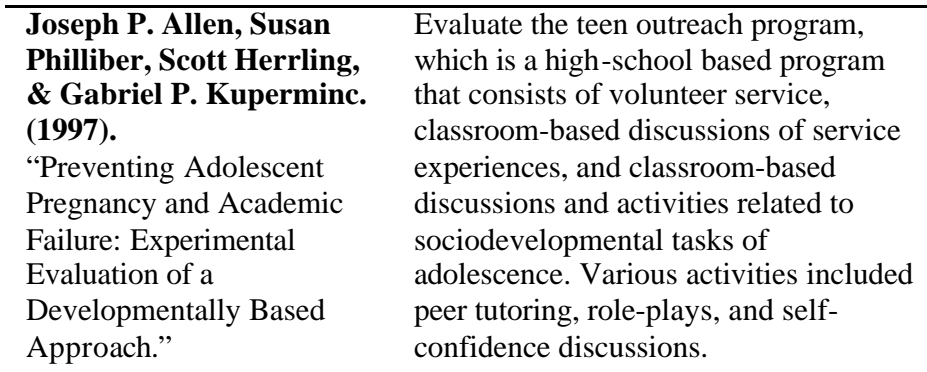

The evaluation involved 695 high

school students in 25 sites nationwide from 1991 through 1995. Random assignment to either a treatment or control group was done, and a preand posttest were conducted on both groups.
The 25 sites nationwide were selected via random assignment. Students ranged in grade level from 9th through 12th. The average grade level was 10 th grade.
Findings show that rates of pregnancy, school failure, and suspension were much lower in the treatment group than the control group when demographic characteristics were controlled. No significant effects were found related to program intensity, but there was a significant inverse effect of course failure on the number of volunteer hours worked (i.e., those who did more volunteer hours were less likely to fail a course). 


\section{APPENDIX A, PART 1: YOUTH DEVELOPMENT IMPACTS ON TEENS' SEXUAL BEHAVIOR}

(CONT'D)

Citation

Purpose of Study
Research/Evaluation Design

The study is part of a longitudinal study of

consenting 5th graders in 18 public schools in high crime areas of Seattle, Washington. Thi particular study involved a full intervention group (grades 1-6), a late intervention group (grades 5-6), and a control group, totaling almost 600 students. Students in all three groups did not differ much in gender, ethnicity, or poverty level.

This was a matched school quasi-experimental design.

\author{
Community and Setting Summary of Findings
}

This study involves an equal proportion of boys and girls in the 5th grade who are primarily from low-income households in Seattle, Washington. More than half of the participants were on the national school lunch and school breakfast program. The majority of the participants behaviors. The components include teaching, and a curriculum for both were white. were significantly more bonded to from ages 13 to 18 for the late problem behaviors) in the full intervention group. These findings

Students in the full intervention group school than the control group at ages 13 and 18 . School bonding declined intervention and control groups. School bonding was significantly related to reduced sexual activity (among other suggest that social development interventions through the elementary school years have a positive long-term effect on school bonding and therefore show promise for preventing problem behaviors at age 18 .

\section{Susan Philliber,} Jacqueline Williams

Kaye, Scott Herrling, \& Emily West.

(2002).

"Preventing Pregnancy and Improving Health Care Access among Teenagers: an Evaluation of the Children's Aid Society-Carrera Program."
The objective of this study is to evaluate the Carrera program, which is a yearround after-school program started in 1984 with a comprehensive youth development orientation. The program consists of seven components: (1) job club, (2) academic performance assistance, (3) comprehensive family life and sexuality education, (4) art activities, (5) sport activities, (6) mental health care, and (7) medical care.

\section{Six agencies in NYC randomly assigned a} total of 600 adolescents to the Carrera program $(\sim 300$ students in each the treatment and control groups). The two groups were tracked for three years-more than threequarters of participants stayed in the program during all these three years. Students were eligible if they were not already involved in an after-school program, were aged 13 through

15 , and were not pregnant and were not parents. Recruitment strategies included school outreach, flyers, and contacting families. Baseline data were collected from February through April 1997.

\author{
Each of the six NYC sites \\ included 100 students aged 13 \\ through 15 (totaling 600 \\ students across all sites) \\ Program activities ran every \\ weekday for three hours per \\ day. The majority of the \\ students were black and \\ Hispanic, many of whom came \\ from economically \\ disadvantaged families

\section{Findings suggest that female} \\ participants were much more likely to \\ lower their chance of being sexually \\ active as a result of the Carrera \\ program. Longterm connections to the \\ program prevent teenage pregnancy by \\ improving the girls' sexual literacy and \\ increasing the use of effective \\ contraceptives. However, there were no \\ significant effects on male participants \\ with respect to sexual and reproductive \\ behavior outcomes. Overall, both male \\ and female participants had a higher \\ chance of receiving good primary \\ health care.
}




\section{APPENDIX A, PART 1: YOUTH DEVELOPMENT IMPACTS ON TEENS' SEXUAL BEHAVIOR (CONT'D)}

Citation

Purpose of Study approach to reducing pregnancy among high-risk youth. The program's

intervention components include (1) education and skills building; (2) mentorship; and (3) advocacy. Each of the seven sites varied in objectives, target populations, setting, and types of project staff.

Client-Centered

Programs to Preven
Evaluate the effects of a client-centered
Research/Evaluation Design through 13) were served by four projects, and three projects served 690 older adolescents (ages 14 through 17). Participants were randomized to treatment and control groups. Data were collected during the pre- and posttesting periods, and were collected only in group settings.
A total of 1,042 early adolescents (ages 9

Community and Setting Summary of Findings

The targeted population was adolescents ages 9 through 17 in seven communities in

Washington state. The majority of the participants were white and female. Each of the seven

Adolescent Pregnancy." sites varied in target population age and setting.
Results varied across the seven communities. One site found that engage in risky sexual behavior.

students who received more hours of services per year were less likely to intend to have sex, and another site reported that the participants in the treatment group were less likely to Overall, this client-centered program showed no effects on factors that place children and adolescents at risk of becoming pregnant, such as parentchild communication and sexua values.

Lydia O'Donnell, Ann Stueve, Alexi San Doval, Richard Duran, Deborah Haber,

\section{Rebecca Atnafou,}

Norma Johnson, Uda

Grant, Helen Murray, Greg Juhn, Julia Tang,

\section{\& Patricia Piessens.}

(1999).

The Effectiveness of the

Reach for Health

Community Youth

Service Learning

Program in Reducing

Early and Unprotected

Sex among Urban Middle

School Students.
Evaluate the effectiveness of a

community youth service program called

Reach for Health in reducing sexual risk behaviors urban youth. Activities were designed to promote or improve social skills and behaviors, though each assignment is grade- or age-appropriate. The classroom-based curriculum was based on one of the most widely used comprehensive health curricula in the U.S. The curriculum focused on (1) drug and alcohol use, (2) violence, and (3) risky sexual behavior. Participants spent three hours per week in a community placement such as nursing homes, fullservice health clinics, and child care centers.
A total of 1,061 students at two large public urban middle schools were surveyed at baseline and six months after completing the program. Students at one school were randomly assigned by classroom to receive either the reach for health community youth service program plus curriculum or the reach for health classroom curriculum only. Students at the second middle school served as the control group. The school sites were very similar in that they have a large and almost exclusively minority student body.

\section{The targeted population wa} black and Hispanic urban adolescents.
Participants in the treatment group who received the curriculum plus engaged in community youth service activities showed significant improvement in using contraceptives during most recent sex at the six-month follow-up (among those who reported recently engaging in sex). Participants who received the curriculum only also showed improvements in these areas at the follow-up interview. The greatest effect was among 8th graders who received the most intensive service program. Special-education students appeared to benefit the most from the curriculum-only intervention. 


\section{APPENDIX A, PART 1: YOUTH DEVELOPMENT IMPACTS ON TEENS' SEXUAL BEHAVIOR \\ (CONT'D)}

\begin{tabular}{|c|c|c|c|c|}
\hline Citation & Purpose of Study & Research/Evaluation Design & Community and Setting & Summary of Findings \\
\hline $\begin{array}{l}\text { Gary Walker \& } \\
\text { Frances Vilella-Velez. } \\
\text { (1992). } \\
\text { The Summer Training } \\
\text { and Education Program } \\
\text { (STEP) from Pilot } \\
\text { through Replication and } \\
\text { Postprogram Impacts. }\end{array}$ & $\begin{array}{l}\text { Evaluate the summer training and } \\
\text { education (STEP) program. The } \\
\text { program was designed to stem summer } \\
\text { learning loss among at-risk youth by } \\
\text { providing paid summer work and } \\
\text { remedial education. Key components } \\
\text { include remediation ( } 90 \text { hours), life } \\
\text { skills and opportunities (one half-day } \\
\text { per week), work experience (five half- } \\
\text { days per week), and school-year } \\
\text { support. }\end{array}$ & $\begin{array}{l}\text { Step was implemented in five cities in the } \\
\text { summer of } 1985 \text { (Boston, Fresno, San Diego, } \\
\text { Portland, and Seattle). The evaluation was a } \\
\text { random assignment in which the treatment } \\
\text { group received all key components, and the } \\
\text { control group was offered a summer job only. } \\
\text { Eligible students (ages } 14-15 \text { who are } \\
\text { economically and educationally disadvantaged) } \\
\text { received two summers and an intervening } \\
\text { school year of services. Program outcomes } \\
\text { from postprogram interviews with both groups } \\
\text { and school transcripts were analyzed. }\end{array}$ & $\begin{array}{l}\text { The program targeted low-income } \\
\text { youth performing below grade level. } \\
\text { Most were in the 8th or 9th grade } \\
\text { but had reading and math levels far } \\
\text { below their grade level. The } \\
\text { majority of the participants were } \\
\text { black. Almost one-third of the } \\
\text { participants had been held back at } \\
\text { least one grade level, half of them } \\
\text { lived in single-parent households, } \\
\text { and almost half of them were } \\
\text { sexually active. Of those who } \\
\text { reported being sexually active, } 75 \\
\text { percent of them did not use } \\
\text { contraceptives. }\end{array}$ & $\begin{array}{l}\text { Findings varied considerably across the } \\
\text { five sites. Compared with the control } \\
\text { group, the treatment group performed } \\
\text { significantly better in math and } \\
\text { reading, consumed less alcohol, and } \\
\text { increased their knowledge of birth } \\
\text { control methods. About } 3.5 \text { years after } \\
\text { enrollment into the program, the } \\
\text { treatment and control groups were } \\
\text { comparable in educational measures, } \\
\text { employment, drug use, and pregnancy. } \\
\text { There did not seem to be any } \\
\text { significant improvement in reducing } \\
\text { teenage pregnancies. }\end{array}$ \\
\hline $\begin{array}{l}\text { Andrew Hahn, Tom } \\
\text { Leavitt, \& Paul Aaron. } \\
\text { (1994). } \\
\text { "Evaluation of the } \\
\text { Quantum Opportunities } \\
\text { Program: Did the } \\
\text { Program Work?" }\end{array}$ & $\begin{array}{l}\text { Evaluate the effectiveness of the } \\
\text { Quantum Opportunities program on } \\
\text { school graduation, teenage pregnancy, } \\
\text { and violent behavior. The program is } \\
\text { an intensive, multicomponent } \\
\text { intervention program for } \\
\text { disadvantaged teenagers during their } \\
\text { four years of high school. Program } \\
\text { components include academic help, } \\
\text { community service, and financial } \\
\text { incentives. }\end{array}$ & $\begin{array}{l}\text { Fifty students in five sites (Philadelphia, San } \\
\text { Antonio, Saginaw, Oklahoma City, and } \\
\text { Milwaukee) were recruited for this study } \\
\text { (totaling more than } 600 \text { high school students). } \\
\text { The program provides approximately } 250 \text { hours } \\
\text { per year of intensive educational assistance, } \\
\text { community service, and developmental } \\
\text { activities. Some activities are offered year- } \\
\text { round. Service is delivered via a counselor who } \\
\text { is assigned to a specific group for the four years } \\
\text { throughout high school. About } 85 \text { percent of the } \\
\text { original group were available for the post-high } \\
\text { school follow-up. }\end{array}$ & $\begin{array}{l}\text { The target population includes 9th } \\
\text { graders from primarily low-income } \\
\text { families. The setting of these } \\
\text { activities varies from community } \\
\text { agencies to schools to homes. }\end{array}$ & $\begin{array}{l}\text { After two years of inclusion in the } \\
\text { program, the treatment group averaged } \\
\text { much higher than the control group on } \\
\text { all } 11 \text { academic and functional skills. } \\
\text { Participation decreased the chances of } \\
\text { teenage pregnancy, dropout, and } \\
\text { fatherhood among boys. The } \\
\text { Philadelphia site was the most } \\
\text { successful-it is the only site with } \\
\text { statistically significant results. This } \\
\text { could be due to the larger sample of } \\
\text { children available at the Philadelphia } \\
\text { site. }\end{array}$ \\
\hline
\end{tabular}




\section{APPENDIX A, PART 2: YOUTH DEVELOPMENT IMPACTS ON THE ANTECEDENTS OF FIRST SEX}

\begin{tabular}{|c|c|c|c|c|}
\hline Citation & Purpose of Study & Research Design & Community and Setting & Summary of Findings \\
\hline $\begin{array}{l}\text { Development Studies } \\
\text { Center (2002). } \\
\text { Summary of Evaluation } \\
\text { Findings on the Child } \\
\text { Development Project. }\end{array}$ & $\begin{array}{l}\text { Assess the impact of the Child } \\
\text { Development Project, a three-year } \\
\text { school-based intervention with } \\
\text { community, school, and home-based } \\
\text { components for families with children in } \\
\text { 3rd through 6th grade. YD components } \\
\text { included parent/child bonding activities, } \\
\text { community-building projects, peer } \\
\text { mentoring, and teacher training. }\end{array}$ & $\begin{array}{l}\text { Quasi-experimental research design } \\
\text { using two demonstration schools and } \\
\text { two comparison schools in six school } \\
\text { districts across the United States. } \\
\text { Annual spring assessments of drug use } \\
\text { and delinquency collected through } \\
\text { group-administered questionnaires to a } \\
\text { sample of } 1,645 \text { students. School } \\
\text { attachment, mental health, and social } \\
\text { skills were also measured annually for } \\
\text { the three years of the intervention. No } \\
\text { postintervention follow-up. }\end{array}$ & $\begin{array}{l}\text { School setting. Study schools were } \\
\text { located in two large urban districts, } \\
\text { one small urban district, one suburban } \\
\text { district, and one rural district. Student } \\
\text { populations varied greatly across these } \\
\text { schools in ethnic composition, school- } \\
\text { lunch receipt, average achievement, } \\
\text { and limited English proficiency. }\end{array}$ & $\begin{array}{l}\text { After three years of program } \\
\text { implementation, program participants } \\
\text { in five high-implementing schools } \\
\text { experienced greater school attachment, } \\
\text { stronger academic motivation, and } \\
\text { other improvements in academic- } \\
\text { related measures than students in the } \\
\text { control schools. Participants also } \\
\text { exhibited better conflict resolution } \\
\text { skills, reduced delinquency and use of } \\
\text { alcohol, and more frequent altruistic } \\
\text { behavior. }\end{array}$ \\
\hline $\begin{array}{l}\text { Joseph P. Tierney \& } \\
\text { Jean Baldwin Grossman. } \\
(\mathbf{2 0 0 0 )} \text {. } \\
\text { "Making a Difference: An } \\
\text { Impact Study of Big } \\
\text { Brothers Big Sisters" }\end{array}$ & $\begin{array}{l}\text { Assess the impact of the Big Brothers } \\
\text { Big Sisters (BBBS) program, a carefully } \\
\text { structured national mentoring program } \\
\text { designed to create supportive one-to-one } \\
\text { relationships among adult volunteers and } \\
\text { youth living in single-parent households. } \\
\text { Mentors and youth agree to meet two to } \\
\text { four times per month for at least one year. } \\
\text { The program requires numerous } \\
\text { mentoring standards including mentor } \\
\text { screening and training, matching } \\
\text { requirements, and monitoring. }\end{array}$ & $\begin{array}{l}\text { Experimental evaluation of } 1,138 \\
\text { youth between the ages of } 10 \text { and } 16 \\
\text { who applied to the BBBS program in } \\
1992 \text { and } 1993 \text {. Approximately half } \\
\text { were randomized into the program and } \\
\text { half were put on a waiting list. The } \\
1,138 \text { were interviewed before being } \\
\text { randomly assigned. After } 18 \text { months, } \\
\text { the remaining } 959 \text { study participants } \\
\text { (after attrition) were reinterviewed. }\end{array}$ & $\begin{array}{l}\text { Nationwide community-based } \\
\text { program. Eight of the } 500 \text { BBBS local } \\
\text { agencies were selected for their } \\
\text { geographic diversity and large active } \\
\text { caseload and waiting list. }\end{array}$ & $\begin{array}{l}\text { After } 18 \text { months in the program } \\
\text { number of protective factors were } \\
\text { strengthened. These included improved } \\
\text { school attendance, performance and } \\
\text { attitudes toward completing } \\
\text { schoolwork, and improved peer and } \\
\text { family relationships. }\end{array}$ \\
\hline
\end{tabular}




\section{APPENDIX A: PART 2: YOUTH DEVELOPMENT IMPACTS ON THE ANTECEDENTS OF FIRST SEX (CONT'D)}

Citation

Knowlton Johnson, Ted

Strader, Michael

Berbaum, Denise Bryant,

Greg Bucholtz, David

Collins, \& Tim Noe

(1996).

Reducing Alcohol and

Other Drug Use by

Strengthening Community,

Family and Youth

Resiliency: An Evaluation

of the Creating Lasting

Connections Program,

summarized in Catalano et

al. (2002).

Stephen P. Schinke,

Gilbert J. Botvin, J. E.

Trimble, Mario A.

Orlandi, Lewayne D.

Gilchrist, \& V.S.

Locklear. (1988).

"Preventing Substance

Abuse Among American-

Indian Adolescents: A

Bicultural Competence

Skills Approach."

\section{W. Andrews, L. H.}

Soberman, and T. J.

Dishion. (1995). "The

Adolescent Transitions

Program for High-Risk

Teens and Their Parents:

Toward a School-Based

Intervention."
Purpose of Study

The purpose of this study was to

evaluate the Creating Lasting

Connections program, a five year

demonstration project designed to

reduce the frequency of drug use and

abuse among $12-14$ year-olds. YD

components included community

building and the development of family

management and communication skills.

Family skills focused more on the

parents than youth (parents received 15

hours of training)
Research Design

Experimental design where recruited

amilies were randomly assigned to the

program or control conditions. Ninety-

seven parents and 120 youth were in the

sample. Data were collected at baseline, six

to seven months after parent/youth training,

and one year after initiation. Study subjects

were asked questions from the Personal

Experience Inventory (including alcohol

and substance abuse questions) and a series

of communication skill items.
Community and Setting Summary of Findings

Church-based program set in five Participants in the program were

"church communities" in

Louisville, Kentucky.

more likely to use community

services as needed when family problems arose and to perceive an action accomplished something

helpful. While there was no

significant effect on the reduction of substance abuse (SA) overall, SA

was reduced among children whose parents increased their knowledge of SA and where parent/youth conflict decreased.

The purpose of this study was to evaluate the Bicultural Competence Skills program, designed to help Native American children develop a positive identity and become socially competent in two cultures. YD components included peer-based cultural strategies and communication and coping strategies associated with bicultural efficacy.

The purpose of this study was to evaluate the Adolescent Transitions Program, a parent and youth training program for substance abuse and problem behavior prevention. Parents were taught family management skill including prosocial fostering, limit setting, and problem solving. Youth 10 14 were assisted with self-regulation skills including goal setting and problem solving.
Experimental pretest/posttest with followrandomly divided into prevention and control group conditions. Study participants were assessed at baseline, immediately following the program, and six months after the program. up. Subject were voluntarily recruited and
Two Native American reservations in western Washington state.
Program participants had significantly higher ratings of selfcontrol, assertiveness, and the ability to generate alternative suggestions to peer pressure to use drugs. Actual alcohol and drug use also declined and this persisted through six-month follow-up.
Experimental pretest/posttest design. Very poor parents (most European-American) were recruited, and if their child was determined to be at-risk, the parent was assigned to one of four intervention conditions (parent focus, teen focus, selfdirected change and control). Initial sample size was 158 at baseline and 143 at assessment, and each treatment group consisted of only seven to eight families. Post-participation assessments consisted of behavior inventories, audiotaped problem
Program was implemented in two settings - one school setting and one community mental health setting.
Both the parent and teen treatments were shown to be effective at reducing parent/child conflict and negative family events. The parent component, but not the teen component positively affected student p roblem behavior, at least in the short term. 
solving scenarios and videotaped problem

solving sessions.

\section{APPENDIX A: PART 2: YOUTH DEVELOPMENT IMPACTS ON THE ANTECEDENTS OF FIRST SEX (CONT'D)}

\section{Jose A. Cardenas, Maria Robledo Montecel, Josie \\ D. Supik, \& Richard J. \\ Harris. (1992). "The Coca- \\ Cola Valued Youth \\ Program: Dropout \\ Prevention Activities for \\ At-Risk Students." \\ The purpose of this study was to evaluate the Valued Youth Partnership Program, a comprehensive youth development program designed to improve self-concept among Hispanic English-language learners (predominately foreign born or the children of foreign-born parents). Other goals of the program included a reduction in dropout rates and truancy, and disciplinary referrals. YD activities included increased recognition for accomplishments, tutoring (30 sessions per year), and parental involvement in student activities.}

\section{JoAnne L. Pedro-Carroll \& Emory L. Cowen \\ (1985). "The Children of \\ Divorce Intervention \\ Program: An Investigation \\ of the Efficacy of a School- \\ Based Prevention \\ Program."}

Evaluate the Children of Divorce Intervention Program, a 10-session program to assist children of divorced or separated parents in grades 3-6. YD components include group cohesion and trust exercises, activities to help manage anger and anxiety, and to help with decisionmaking and problem solving.

Robert E. Slavin, Nancy A. Madden, Lawrence J. Dolan, Barbara A. Wasik, Steven Ross, Lana Smith, and Marcella Dianda. (1996). "Success for All: A Summary of Research."
Evaluate the Success for All program, which focuses on academic success through developing a child's selfrespect and self-efficacy. YD components include tutoring and parenting skills workshops, in addition to frequent assessments and medical services.
Quasi-experimental design. Sample consisted of 101 treatment students and 93 comparison students with an average age of 12. Postprogram data were collected after one year and two years in the program on self-concept, and quality of school life. grades, disciplinary action, absenteeism,

School-based program administered in two public

schools in San Antonio, Texas.

\section{Experimental design with children} randomly assigned to experimental and control conditions within schools. Posttests were conducted two weeks after the session had ended. No follow-up was conducted. Study participants were assessed using teacher ratings.

Four suburban schools.
Program was shown to reduce dropout and improve grades. Selfconcept and attitudes toward school also improved after the first year, but eveled off in the second year (and were no different that comparison group)
Quasi-experimental using a matched comparison group.

School-based program

Program participants showed significant results for reading scores at all grade levels. The impact of the program was twice as large for students in the lowest 25 percent of the grade.
Teacher ratings for program participants indicated that they had ower anxiety, fewer learning programs [[problems?]], and were better adjusted in the classroom that nonstudy participants

services.


Appendix B: Document Review Protocol

Name

Program Name

Address

\section{INFORMATION THAT WAS REVIEWED:}

\section{UI reviewer:}

\section{CRITIQUE OF INFORMATION:}

OAPP project officer's ranking of program:

\section{OAPP project officer:}

Year of report:

\section{ORGANIZATIONAL CHARACTERISTICS}

Orga nization mission:

Organization type:

Funding status:

Amount of funding:

Project director:

Personnel information:

\section{DEMOGRAPHICS OF TARGET AREA AND POPULATION}

- Target area

- Target population

\section{Program characteristics: Project [name]}

- Mission:

- Program intervention(s) and setting(s):

- Length of intervention:

- Presence of positive YD goals (Catalano et al. 2002; 2004) for definitions of constructs, see p. 2 of Codebook) [Yes/No]:

- Promotes bonding

- Parent-child communication

- Fosters resilience

- Promotes social competence

- Promotes emotional competence

- Promotes cognitive competence

- Promotes behavioral competence

- Promotes moral competence

○ Fosters self-determination 
○ Fosters spirituality

- Fosters self-efficacy

- Fosters clear and positive identity

- Fosters belief in the future

- Provides recognition for positive behavior

- Provides opportunities for prosocial involvement

- Fosters prosocial norms

- YD intervention components:

○ Youth intervention:

- Curriculum for youth intervention:

-

○ Parent intervention:

- Curriculum for parent intervention:

$\bullet$

- Abstinence education intervention components:

○ Youth intervention:

- Curriculum for youth intervention:

$\bullet$

○ Parent intervention:

- Curriculum for parent intervention:

- Moderating factors that may impact intervention outcomes [as interpreted by UI reviewer

- Goals and progress: [as stated in documents available] [indicate whether long- or shortterm goals, if stated in documents]

○ Goals and objectives

- Progress

Challenges and proposed solutions [as stated in documents available]

Evaluation methods

- Evaluator:

- Evaluation research questions:

O

- Evaluation model:

○ Type of evaluation

- Comparison/control group

- Pre- and posttesting

- Follow-up [other than posttesting]

- Longitudinal

- Evaluability assessment [YES/NO]

- Knowledge

- Attitude

- Behavior intention

- Behavior

- MIS: [what type of system, process] 
- Evaluation instruments:

○ Type of data being collected: [surveys, questionnaires, focus groups observations, etc.]

- Who is responsible for collecting data:

- Findings for program year: [as stated in documents available]

Ranking of program

- Dimension \#1: Intensity of each intervention

○ Duration (score):

- Frequency of meetings (score):

- Training of project and evaluation staff (score):

- Dimension \#2: Presence of constructs

○ Presence and achievement of constructs (score):

- Dimension \#3: Evaluability assessment

○ Design (score):

- Ability to measure outcomes (score):

- Follow-up tests beyond immediate posttesting (score): 


\section{APPENDIX C}

\begin{tabular}{|c|c|}
\hline $\begin{array}{l}\text { Positive Youth } \\
\text { Development } \\
\text { Objective }\end{array}$ & Enrichment Activities \\
\hline Promotes Bonding & $\begin{array}{l}\text { Mentoring with teachers, community members, high school or } \\
\text { college students, etc. } \\
>\text { Peer-mentoring program in which teens are selected to act as role } \\
\text { models for other students } \\
>\text { Gender-specific support groups are offered twice a month to } \\
\text { provide youth with the opportunity to discuss events, team-building } \\
\text { activities, and preadolescent developmental changes }\end{array}$ \\
\hline $\begin{array}{l}\text { Promotes Parent- } \\
\text { Child } \\
\text { Communication }\end{array}$ & $\begin{array}{l}>\text { Parents are encouraged to chaperone field trips and special events } \\
\text { Annual or special events are scheduled for parental participation } \\
\text { (i.e. Family Fishing Day, Family Fun Day, Family Dinner Night) } \\
>\text { Parent nights are hosted once a month to provide parents with } \\
\text { information how to communicate with their youth, review the } \\
\text { curriculum, and provide update and feedback on their youth's } \\
\text { progress } \\
>\text { Classroom-based activities for children and their parents to } \\
\text { encourage better communication and reduce conflict } \\
>\text { Participants are given exercises to take home to do with parents } \\
>\text { Monthly telephone calls are made to parents in the evenings to } \\
\text { update them } \\
\text { "Padres at the Park" is a male involvement event designed for men } \\
\text { and their children to participate in communication-building } \\
\text { activities }\end{array}$ \\
\hline Fosters Resilience & $\begin{array}{l}\text { Dances, movie socials, and game nights held in a controlled and } \\
\text { safe environment, allow students to test their resiliency skills with } \\
\text { positive role models present } \\
\text { Theater production and workshops work on resisting peer pressure }\end{array}$ \\
\hline $\begin{array}{l}\text { Promotes Social } \\
\text { Competence }\end{array}$ & $\begin{array}{l}>\text { Students participate in a brief "checking-in" meeting before every } \\
\text { activity } \\
>\text { Small gender segregated group sessions to build healthy friendships } \\
\text { and relationships } \\
>\text { Gender-specific support groups are offered twice a month to } \\
\text { provide youth with the opportunity to discuss events, team-building } \\
\text { activities, and preadolescent developmental changes. } \\
>\text { Students participate in the Advisory Board Committee, where they } \\
\text { help make program decisions }\end{array}$ \\
\hline $\begin{array}{l}\text { Promotes } \\
\text { Emotional } \\
\text { Competence }\end{array}$ & $\begin{array}{l}\text { Sports activities are used to foster self-esteem and confidence as } \\
\text { well as healthy living } \\
\text { Discussion groups and other activities designed to promote self- } \\
\text { confidence and self- growth }\end{array}$ \\
\hline $\begin{array}{l}\text { Promotes } \\
\text { Cognitive }\end{array}$ & $\begin{array}{l}\text { Movie and video presentations featuring life skills messages } \\
>\text { Students participate in the Advisory Board Committee, where they }\end{array}$ \\
\hline
\end{tabular}




\begin{tabular}{|c|c|}
\hline Competence & help make program decisions \\
\hline $\begin{array}{l}\text { Promotes } \\
\text { Behavioral } \\
\text { Competence }\end{array}$ & $\begin{array}{l}\text { Mentoring with adults, teachers, or geographically close } \\
\text { college/high school students to help make better behavioral choices }\end{array}$ \\
\hline $\begin{array}{l}\text { Promotes Moral } \\
\text { Competence }\end{array}$ & $\begin{array}{l}\text { Several programs use community service or service learning to help } \\
\text { improve respect for society } \\
\text { Cultural events: Coordinated arts and cultural programs with } \\
\text { members of the community (music, dance, dance theater, and visual } \\
\text { expressions) } \\
>\text { Traditional language and/or cultural activities (i.e., a Native } \\
\text { American program teaches about the native language, costume, } \\
\text { rituals, etc.) } \\
\text { Service learning summer camp that incorporates recreation and } \\
\text { service-learning activities }\end{array}$ \\
\hline $\begin{array}{l}\text { Fosters Self- } \\
\text { Determination }\end{array}$ & $\begin{array}{l}\text { Focus on financial literacy to help promote self-determination } \\
\text { through an eight-hour financial literacy workshop }\end{array}$ \\
\hline Fosters Spirituality & $\begin{array}{l}\text { Students participate in rite of passage ceremonies and learn about } \\
\text { faith heritage }\end{array}$ \\
\hline $\begin{array}{l}\text { Fosters Self- } \\
\text { Efficacy }\end{array}$ & $\begin{array}{l}\text { Dating program that teaches students to discern character based on } \\
\text { observation of behaviors and stated values } \\
\text { Theater production and workshops work on personal } \\
\text { decisionmaking skills } \\
\text { Dances, movie socials, and game nights, held in a controlled and } \\
\text { safe environment, allow students to make decision with positive } \\
\text { role models present }\end{array}$ \\
\hline $\begin{array}{l}\text { Fosters Clear and } \\
\text { Positive Identity }\end{array}$ & $\begin{array}{l}\text { Small gender-based group sessions where students talk about } \\
\text { development and age-relevant issues }\end{array}$ \\
\hline $\begin{array}{l}\text { Fosters Belief in } \\
\text { the Future }\end{array}$ & $\begin{array}{l}\text { College prepatory program in which participants visit college } \\
\text { campuses and college fairs } \\
\text { Project in which students create, direct, and produce a public access } \\
\text { cable TV show } \\
\text { Academic tutoring offered after school or on weekends to help } \\
\text { improve students' performance in school and improve the outlook } \\
\text { of the future } \\
>\text { Summer internships for participants in which they receive training } \\
\text { in communication, presentations, organizational skills, and the like } \\
\text { and are then required to volunteer at an organization or company } \\
\text { Service- learning activities allow youth to gain skills necessary to } \\
\text { improve their job marketability, while providing services to the } \\
\text { community }\end{array}$ \\
\hline $\begin{array}{l}\text { Provides } \\
\text { Recognition for } \\
\text { Positive Behavior }\end{array}$ & $\begin{array}{l}\text { Students win awards and prizes for above-average participation in } \\
\text { programs and special achievements or accomplishments } \\
>\text { Special events celebrating those students who have taken a pledge } \\
\text { to reduce risk factors in their lives } \\
>\text { At the completion of the curriculum, a community celebration is } \\
\text { held to recognize the students as young adults capable of }\end{array}$ \\
\hline
\end{tabular}




\begin{tabular}{|l|l|}
\hline & \multicolumn{1}{|c|}{ contributing to their families and community } \\
\hline Provides & $>$ Dances \\
Opportunities for & $>$ Movie socials \\
Prosocial & $>$ Game nights \\
Involvement & $>$ Special events \\
& $>$ Advisory Board Committee \\
\hline $\begin{array}{l}\text { Fosters Prosocial } \\
\text { Norms }\end{array}$ & $>$ Community service project reinforced reproductive health (students \\
& $>\begin{array}{l}\text { decorated gift boxes for HIV patients) } \\
\text { provide youth with the opportunity to discuss events, team-building } \\
\end{array}$ \\
& activities, and preadolescent developmental changes \\
\hline
\end{tabular}




\section{APPENDIX D, PART 1: SUMMARY OF DOCUMENT REVIEWS}

\begin{tabular}{|c|c|c|c|c|c|c|c|c|c|c|c|c|c|c|}
\hline \multicolumn{15}{|c|}{ APPENDIX D, PART 1: SUMMARY OF DOCUMENT REVIEW, PROGRAM CHARACTERISTICS } \\
\hline Organization & State & Region & $\begin{array}{l}\text { Grade } \\
\text { Served by } \\
\text { Program }\end{array}$ & $\begin{array}{l}\text { Gender } \\
\text { Served }\end{array}$ & Race & Setting & Duration & Frequency & Training & Design & Outcomes & Followup & Chal1 & Chal2 \\
\hline Be'er Hagolah Institutes & NY & East & $3-12$ & Girls & Russian & Summer & Medium & Missing & Missing & \begin{tabular}{|c|} 
Random \\
Experimental \\
Design
\end{tabular} & $\begin{array}{l}\text { Actual } \\
\text { Behavior }\end{array}$ & No & $\begin{array}{c}\text { LowiNo } \\
\text { Parental } \\
\text { Involvement }\end{array}$ & Missing \\
\hline Boston Medical Center & MA & East & $7-8$ & $\begin{array}{l}\text { Boys and } \\
\text { Girls }\end{array}$ & $\begin{array}{l}\text { Mostly } \\
\text { Black }\end{array}$ & Mixed & Medium & Medium & Yes & $\begin{array}{c}\text { Quasi- } \\
\text { Experimental } \\
\text { Design }\end{array}$ & $\begin{array}{l}\text { Behavioral } \\
\text { Intentions }\end{array}$ & No & $\begin{array}{l}\text { Recruitment/ } \\
\text { Retertion of } \\
\text { Youth } \\
\text { Participants } \\
\end{array}$ & Evaluation Design \\
\hline Builders for the Farmily \& Youth & NY & East & $6-8$ & $\begin{array}{c}\text { Boys and } \\
\text { Girls }\end{array}$ & $\begin{array}{l}\text { Mostly } \\
\text { Latino }\end{array}$ & After School & Medium & Medium & Yes. & \begin{tabular}{|c|} 
All Other \\
Types of \\
Design
\end{tabular} & $\begin{array}{l}\text { Actual } \\
\text { Behavior }\end{array}$ & No & Missing & Missing \\
\hline Catholic Charities of Onondaga $\mathrm{C}_{0}$ & NY & East & 7 & $\begin{array}{c}\text { Boys and } \\
\text { Girls }\end{array}$ & $\begin{array}{l}\text { Mostly } \\
\text { Black }\end{array}$ & After School & Medium & Medium & Yes & $\begin{array}{c}\text { Random } \\
\text { Experimertai } \\
\text { Design }\end{array}$ & $\begin{array}{c}\text { Actual } \\
\text { Behavior }\end{array}$ & Yes & $\begin{array}{l}\text { Recruitment/ } \\
\text { Retention of } \\
\text { Youth } \\
\text { Participants } \\
\end{array}$ & Evaluation Design \\
\hline $\begin{array}{l}\text { Crozer-Chester Medical Center } \\
\text { (Leadership 101) }\end{array}$ & $P A$ & East & $6-8$ & $\begin{array}{l}\text { Boys and } \\
\text { Girls }\end{array}$ & $\begin{array}{l}\text { Mostly } \\
\text { Elack }\end{array}$ & After School & Medium & Missing & Yes & $\begin{array}{c}\text { Random } \\
\text { Experimental } \\
\text { Design }\end{array}$ & $\begin{array}{l}\text { Behavioral } \\
\text { Intentions }\end{array}$ & No & $\begin{array}{c}\text { High Staff } \\
\text { Turnover Ratel } \\
\text { Inadequate } \\
\text { Number of Staff }\end{array}$ & Evaluation Design \\
\hline $\begin{array}{l}\text { Crozer-Chester Medical Center } \\
\text { (Successtul Passages) }\end{array}$ & $\mathrm{PA}$ & East & 8 & $\begin{array}{l}\text { Boys and } \\
\text { Girls }\end{array}$ & $\begin{array}{l}\text { Mostly } \\
\text { Black }\end{array}$ & In School & Medium & Missing & Yes & \begin{tabular}{|c|} 
Random \\
Experimental \\
Design
\end{tabular} & $\begin{array}{l}\text { Behavioral } \\
\text { Intertions }\end{array}$ & No & Other & Missing \\
\hline Educ for Children Fanilies \& Youth & NY & East & $6-8$ & $\begin{array}{l}\text { Boys and } \\
\text { Girls }\end{array}$ & Missing & After School & Missing & Missing & Missing & Missing & Missing & Missing & $\begin{array}{c}\text { No Challenges } \\
\text { Reported }\end{array}$ & Missing \\
\hline Mercy Hospital of Pittsburgh & $\mathrm{PA}$ & East & $6-12$ & $\begin{array}{l}\text { Boys and } \\
\text { Girls }\end{array}$ & $\begin{array}{l}\text { Mostly } \\
\text { Elack }\end{array}$ & Mixed & Low & Low & Yes & $\begin{array}{l}\text { All Other } \\
\text { Types of } \\
\text { Design }\end{array}$ & $\begin{array}{l}\text { Behavioral } \\
\text { Intentions }\end{array}$ & No & $\begin{array}{c}\text { High Staff } \\
\text { Turnover Rater } \\
\text { Inadequate } \\
\text { Number of Staff }\end{array}$ & $\begin{array}{c}\text { Difficulty } \\
\text { Establishing Stable } \\
\text { School/Conmunity } \\
\text { Partnership }\end{array}$ \\
\hline St. Luke's Roosevett Hospital & NY & East & $5-6$ & $\begin{array}{l}\text { Boys and } \\
\text { Girls }\end{array}$ & Missing & Summer & Low & Low & Yes: & \begin{tabular}{|c|} 
Quasi- \\
Experimental \\
Design
\end{tabular} & $\begin{array}{l}\text { Actual } \\
\text { Behavior }\end{array}$ & No & $\begin{array}{c}\text { No Challenges } \\
\text { Reported }\end{array}$ & Missing \\
\hline To Our Children's Future with Health & $\mathrm{PA}$ & East & $6-8$ & $\begin{array}{l}\text { Boys and } \\
\text { Girls }\end{array}$ & $\begin{array}{l}\text { Mostly } \\
\text { Black }\end{array}$ & Summer & Low & Low & Yes. & $\begin{array}{c}\text { Random } \\
\text { Experimental } \\
\text { Design }\end{array}$ & $\begin{array}{l}\text { Actual } \\
\text { Behavior }\end{array}$ & No & $\begin{array}{l}\text { Recruitment/ } \\
\text { Retention of } \\
\text { Youth } \\
\text { Participants }\end{array}$ & $\begin{array}{l}\text { High Staff } \\
\text { Turnover Rate/ } \\
\text { Inadequate } \\
\text { Number of Staff }\end{array}$ \\
\hline
\end{tabular}




\begin{tabular}{|c|c|c|c|c|c|c|c|c|c|c|c|c|c|c|}
\hline \multicolumn{15}{|c|}{$\begin{array}{l}\text { APPENDIX D, PART 1: SUMMARY OF DOCUMENT REVIEW, PROGRAM CHARACTERISTICS } \\
\text { Continued }\end{array}$} \\
\hline Organization & State & Region & $\begin{array}{l}\text { Grade } \\
\text { Served by } \\
\text { Program }\end{array}$ & $\begin{array}{l}\text { Gender } \\
\text { Served }\end{array}$ & Race & Setting & Duration & Frequency & Training & Design & Outcomes & Followup & Chal1 & Chal2 \\
\hline University of Maryland Baltimore & MD & East & $3-9$ & $\begin{array}{c}\text { Boys and } \\
\text { Girls }\end{array}$ & Mixed & 9 & Medium & Medium & Yes & \begin{tabular}{|c|c|c|c|c|}
$\begin{array}{c}\text { Random } \\
\text { Experimental } \\
\text { Design }\end{array}$ \\
\end{tabular} & $\begin{array}{c}\text { Actual } \\
\text { Behavior }\end{array}$ & Yes & $\begin{array}{l}\text { Recruitment/ } \\
\text { Reterition of } \\
\text { Youth } \\
\text { Participants } \\
\end{array}$ & $\begin{array}{c}\text { Difficulty } \\
\text { Establishing Stable } \\
\text { Schoolicommunity } \\
\text { Partnership }\end{array}$ \\
\hline YMCA of Cumberland, MD & MD & East & $8-9$ & $\begin{array}{c}\text { Boys and } \\
\text { Girls }\end{array}$ & $\begin{array}{l}\text { Mostly } \\
\text { White }\end{array}$ & Other & Low & Low & Yes & \begin{tabular}{c|} 
All Other \\
Types of \\
Design
\end{tabular} & Attitudes & No & \begin{tabular}{|c|} 
High Staff \\
Turnover Ratei \\
Inadequate \\
Number of Staff \\
\end{tabular} & Evaluation Design \\
\hline Eowling Green State Univ & $\mathrm{OH}$ & Midwest & $5-8$ & $\begin{array}{c}\text { Eoys and } \\
\text { Girls }\end{array}$ & $\begin{array}{l}\text { Mostly } \\
\text { White }\end{array}$ & Mixed & Missing & Missing & Yes & \begin{tabular}{|c|} 
Random \\
Experimental \\
Design
\end{tabular} & $\begin{array}{c}\text { Actual } \\
\text { Behavior }\end{array}$ & Yes & $\begin{array}{c}\text { Evaluation } \\
\text { Design }\end{array}$ & Missing \\
\hline Catholic Social Sves of Miami Valley & $\mathrm{OH}$ & Midwest & $5-12$ & $\begin{array}{c}\text { Boys and } \\
\text { Girls }\end{array}$ & $\begin{array}{l}\text { Mostly } \\
\text { Black }\end{array}$ & After School & Medium & Low & Yes & $\begin{array}{c}\text { Quasi- } \\
\text { Experimental } \\
\text { Design }\end{array}$ & $\begin{array}{l}\text { Behavioral } \\
\text { Intentions }\end{array}$ & No & \begin{tabular}{|c|} 
High Staff \\
Turnover Ratel \\
Inadequate \\
Number of Staff \\
\end{tabular} & $\begin{array}{c}\text { Difficulty } \\
\text { Establishing Stable } \\
\text { Schoolicommunity } \\
\text { Partnership }\end{array}$ \\
\hline Lake Co Health Dept & IL & Midwest & $6-11$ & $\begin{array}{l}\text { Boys and } \\
\text { Girls }\end{array}$ & Latino Only & Mixed & Missing & Missing & Yes & $\begin{array}{c}\text { Random } \\
\text { Experimental } \\
\text { Design }\end{array}$ & $\begin{array}{c}\text { Actual } \\
\text { Behavior }\end{array}$ & No & \begin{tabular}{|l|} 
Recruitment/ \\
Retention of \\
Youth \\
Participants \\
\end{tabular} & $\begin{array}{c}\text { LowNo Parental } \\
\text { Involvement }\end{array}$ \\
\hline Pine Ridge Indian Reservation & $\mathrm{SD}$ & Midwest & $4-9$ & $\begin{array}{c}\text { Boys and } \\
\text { Girls }\end{array}$ & $\begin{array}{c}\text { Native } \\
\text { American }\end{array}$ & Mixed & Missing & Missing & Yes & $\begin{array}{c}\text { Random } \\
\text { Experimental } \\
\text { Design }\end{array}$ & $\begin{array}{l}\text { Behavioral } \\
\text { Intentions }\end{array}$ & No & $\begin{array}{c}\text { Evaluation } \\
\text { Design }\end{array}$ & Missing \\
\hline St. Vincent Mercy Medical Center & $\mathrm{OH}$ & Midwest & $7-12$ & $\begin{array}{c}\text { Boys and } \\
\text { Girls }\end{array}$ & Mixed & In School: & Low & Missing & Yes. & $\begin{array}{c}\text { All Other } \\
\text { Types of } \\
\text { Design }\end{array}$ & $\begin{array}{l}\text { Behavioral } \\
\text { Intentions }\end{array}$ & No & Other & Missing \\
\hline Tri County Right to Life Educ Fdn & $\mathrm{OH}$ & Midwest & $6-12$ & $\begin{array}{c}\text { Boys and } \\
\text { Girls }\end{array}$ & $\begin{array}{l}\text { Native } \\
\text { American }\end{array}$ & After School & Medium & Medium & Missing & $\begin{array}{c}\text { Quasi- } \\
\text { Experimental } \\
\text { Design }\end{array}$ & $\begin{array}{l}\text { Behavioral } \\
\text { Intertions }\end{array}$ & Yes & Other & Missing \\
\hline Wise Women Gathering Place & m & Midwest & $6-8$ & $\begin{array}{c}\text { Boys and } \\
\text { Girls }\end{array}$ & $\begin{array}{c}\text { Native } \\
\text { American }\end{array}$ & Mixed & Low & Medium & Yes & $\begin{array}{c}\text { All Other } \\
\text { Types of } \\
\text { Design }\end{array}$ & $\begin{array}{l}\text { Behavioral } \\
\text { Intentions } \\
\end{array}$ & No & $\begin{array}{c}\text { Evaluation } \\
\text { Design }\end{array}$ & Missing \\
\hline Youth \& Fanily Services & SD & Midwest & $4-9$ & Girls & $\begin{array}{l}\text { White and } \\
\text { Native } \\
\text { American }\end{array}$ & After School & Missing & Missing & No & Missing & Missing & Missing & $\begin{array}{l}\text { LowNo } \\
\text { Parental } \\
\text { Involvement }\end{array}$ & $\begin{array}{c}\text { High Staff } \\
\text { Turnover Ratel } \\
\text { Inadequate } \\
\text { Number of Staff }\end{array}$ \\
\hline Alabarna State University & A.L & South & $6-8$ & $\begin{array}{l}\text { Boys and } \\
\text { Girls }\end{array}$ & Mixed & After School & Low & Low & Yes & $\begin{array}{c}\text { Random } \\
\text { Experimental } \\
\text { Design }\end{array}$ & $\begin{array}{l}\text { Actual } \\
\text { Behavior }\end{array}$ & No & $\begin{array}{c}\text { No Challenges } \\
\text { Reported }\end{array}$ & Missing \\
\hline
\end{tabular}




\begin{tabular}{|c|c|c|c|c|c|c|c|c|c|c|c|c|c|c|}
\hline \multicolumn{15}{|c|}{$\begin{array}{l}\text { APPENDIX D, PART 1: SUMMARY OF DOCUMENT REVIEW, PROGRAM CHARACTERISTICS } \\
\text { Continued }\end{array}$} \\
\hline Organization & State & Region & $\begin{array}{l}\text { Grade } \\
\text { Served by } \\
\text { Program }\end{array}$ & $\begin{array}{l}\text { Gender } \\
\text { Served }\end{array}$ & Race & Setting & Duration & Frequency & Training & Design & Outcomes & Followup & Chal1 & Chal2 \\
\hline Baptist Children's Home Miristries & $T X$ & South & 4-12 & $\begin{array}{l}\text { Boys and } \\
\text { Girls }\end{array}$ & Latino Only & Mixed & High & Medium & Yes. & $\begin{array}{l}\text { All other } \\
\text { Types of } \\
\text { Design }\end{array}$ & $\begin{array}{l}\text { Behavioral } \\
\text { Intentions }\end{array}$ & No & $\begin{array}{l}\text { LowNo } \\
\text { Parental } \\
\text { Involvement }\end{array}$ & $\begin{array}{c}\text { High Staff } \\
\text { Turnover Rater } \\
\text { Inadequate } \\
\text { Number of Staff }\end{array}$ \\
\hline BETA. Center & $\mathrm{FL}$ & South & $4-9$ & $\begin{array}{l}\text { Boys and } \\
\text { Girls }\end{array}$ & Mixed & Mixed & Low & Low & Yes & Missing & $\begin{array}{l}\text { Behavioral } \\
\text { Intentions }\end{array}$ & No & $\begin{array}{l}\text { Recruittinentit } \\
\text { Retention of } \\
\text { Youth } \\
\text { Participants }\end{array}$ & $\begin{array}{l}\text { Loww No Parental } \\
\text { Involvement }\end{array}$ \\
\hline Boat People SOS & $\mathrm{VA}$ & South & $7-12$ & $\begin{array}{l}\text { Boys and } \\
\text { Girls }\end{array}$ & Vietnamese & After School & Low & Low & Missing & $\begin{array}{c}\text { Quasi- } \\
\text { Experimental } \\
\text { Design }\end{array}$ & $\begin{array}{c}\text { Actual } \\
\text { Behavior }\end{array}$ & No & \begin{tabular}{|c|} 
Difficulty \\
Establishing \\
Stable \\
School/Commun \\
ity Partnership
\end{tabular} & Missing \\
\hline Boys and Girls Club of Sarasota & $\mathrm{FL}$ & South & $1-12$ & $\begin{array}{l}\text { Boys and } \\
\text { Girls }\end{array}$ & $\begin{array}{l}\text { White and } \\
\text { Black }\end{array}$ & Mixed & Missing & Missing & Yes & $\begin{array}{l}\text { All Other } \\
\text { Types of } \\
\text { Design }\end{array}$ & Attitudes: & No & Other & Missing \\
\hline Clippers & NC & South & 3-10 & $\begin{array}{l}\text { Boys and } \\
\text { Girls }\end{array}$ & Missing & Summer & Low & High & Missing & Missing & Missing & Missing & Missing & Missing \\
\hline Dallas Independent School District & $T X$ & South & $6-12$ & $\begin{array}{c}\text { Boys and } \\
\text { Girls }\end{array}$ & $\begin{array}{l}\text { Black and } \\
\text { Latino }\end{array}$ & Summer & Missing & Medium & Yes. & $\begin{array}{c}\text { Quasi- } \\
\text { Experimental } \\
\text { Design }\end{array}$ & $\begin{array}{l}\text { Behavioral } \\
\text { Intentions }\end{array}$ & Missing & \begin{tabular}{|c|} 
Difficulty \\
Establishing \\
Stable \\
School/Commun \\
ity Partnership
\end{tabular} & Evaluation Design \\
\hline Dallas Independent School District 2 & $\mathrm{TX}$ & South & $6-12$ & $\begin{array}{l}\text { Boys and } \\
\text { Girls }\end{array}$ & $\begin{array}{l}\text { Elack and } \\
\text { Latino }\end{array}$ & Summer & Medium & Low & Yes & \begin{tabular}{c|} 
Guasi- \\
Experimental \\
Design
\end{tabular} & $\begin{array}{c}\text { Actual } \\
\text { Behavior }\end{array}$ & No & $\begin{array}{c}\text { Evaluation } \\
\text { Design }\end{array}$ & Missing \\
\hline Fitth Ward Enrichment Program & TX & South & $4-12$ & Boys: & $\begin{array}{c}\text { Elack and } \\
\text { Latino }\end{array}$ & Mixed & Medium & Medium & Yes & $\begin{array}{c}\text { Quasi- } \\
\begin{array}{c}\text { Experimental } \\
\text { Design }\end{array}\end{array}$ & $\begin{array}{l}\text { Behavioral } \\
\text { Intentions }\end{array}$ & Yes & $\begin{array}{l}\text { Recruitment/ } \\
\text { Retention of } \\
\text { Youth } \\
\text { Participants }\end{array}$ & $\begin{array}{c}\text { Lowino Parental } \\
\text { Inwolyement }\end{array}$ \\
\hline Healthy Mothers, Heatthy Babies & NC & South & $3-8$ & $\begin{array}{l}\text { Boys and } \\
\text { Girls }\end{array}$ & $\begin{array}{l}\text { Mostly } \\
\text { Black. }\end{array}$ & After School & High & High & Yes & $\begin{array}{c}\text { Random } \\
\text { Experimental } \\
\text { Design }\end{array}$ & $\begin{array}{c}\text { Actual } \\
\text { Behavior }\end{array}$ & No & $\begin{array}{l}\text { LowNNo } \\
\text { Parental } \\
\text { Involvement }\end{array}$ & $\begin{array}{c}\text { High Staff } \\
\text { Turnover Ratei } \\
\text { Inadequate } \\
\text { Number of Staff }\end{array}$ \\
\hline Jackson State University & MS & South & $6-8$ & $\begin{array}{c}\text { Boys and } \\
\text { Girls }\end{array}$ & Mixed & Surmmer & Low & Low & Yes & $\begin{array}{c}\text { Random } \\
\text { Experimental } \\
\text { Design }\end{array}$ & $\begin{array}{l}\text { Actual } \\
\text { Behavior }\end{array}$ & No & Missing & Missing \\
\hline JOVEN & TX & South & $6-10$ & $\begin{array}{l}\text { Boys and } \\
\text { Girls }\end{array}$ & $\begin{array}{l}\text { Mostly } \\
\text { Latino }\end{array}$ & In School & Low & Low & No & $\begin{array}{c}\text { All Other } \\
\text { Types of } \\
\text { Design }\end{array}$ & $\begin{array}{l}\text { Actual } \\
\text { Behavior }\end{array}$ & No & $\begin{array}{l}\text { Recruittrent/ } \\
\text { Retention of } \\
\text { Youth } \\
\text { Participants }\end{array}$ & $\begin{array}{l}\text { LowwNo Parental } \\
\text { Involvement }\end{array}$ \\
\hline Morehouse School of Medicine & AL & South & $3-8$ & $\begin{array}{l}\text { Boys and } \\
\text { Girls }\end{array}$ & $\begin{array}{c}\text { Mostly } \\
\text { Black }\end{array}$ & After School & Missing & Medium & Yes & \begin{tabular}{|c|} 
Random \\
Experimental \\
Design
\end{tabular} & $\begin{array}{l}\text { Actual } \\
\text { Behavior }\end{array}$ & Yes & Other & Missing \\
\hline Public Heatth Authority of Cabarrus & NC & South & $6-8$ & $\begin{array}{l}\text { Boys and } \\
\text { Girls }\end{array}$ & $\begin{array}{l}\text { White and } \\
\text { Black }\end{array}$ & After School & Medium & Medium & Yes & $\begin{array}{c}\text { Random } \\
\text { Experimental } \\
\text { Design }\end{array}$ & $\begin{array}{l}\text { Behavioral } \\
\text { Intentions }\end{array}$ & Yes & $\begin{array}{l}\text { Recruitment/ } \\
\text { Retention of } \\
\text { Youth } \\
\text { Participants }\end{array}$ & Evaluation Desig \\
\hline
\end{tabular}




\begin{tabular}{|c|c|c|c|c|c|c|c|c|c|c|c|c|c|c|}
\hline \multicolumn{15}{|c|}{$\begin{array}{l}\text { APPENDIX D, PART 1: SUMMARY OF DOCUMENT REVIEW, PROGRAM CHARACTERISTICS } \\
\text { Continued }\end{array}$} \\
\hline Organization & State & Region & $\begin{array}{l}\text { Grade } \\
\text { Served by } \\
\text { Program }\end{array}$ & $\begin{array}{l}\text { Gender } \\
\text { Served }\end{array}$ & Race & Setting & Duration & Frequency & Training & Design & Outcomes & Followup & Chal1 & Chal2 \\
\hline Sisterhood Agenda & NC & South & 3-11 & Girls & Elack Only & After School & Missing & Missing & Yes & Missing & Missing & Missing & $\begin{array}{l}\text { Recruitment/ } \\
\text { Reterition of } \\
\text { Youth } \\
\text { Participants } \\
\end{array}$ & Missing \\
\hline Switchboard of Miami & $\mathrm{FL}$ & South & 9 & $\begin{array}{c}\text { Boys and } \\
\text { Girls }\end{array}$ & $\begin{array}{c}\text { Elack and } \\
\text { Latino }\end{array}$ & In School & Medium & Medium & Yes. & $\begin{array}{c}\begin{array}{c}\text { Random } \\
\text { Experimental } \\
\text { Design }\end{array} \\
\end{array}$ & $\begin{array}{l}\text { Behavioral } \\
\text { Intentions }\end{array}$ & Yes & $\begin{array}{c}\text { No Challenges } \\
\text { Reported }\end{array}$ & Missing \\
\hline The Children's Council & SC & South & $9-12$ & $\begin{array}{c}\text { Boys and } \\
\text { Girls }\end{array}$ & $\begin{array}{c}\text { White and } \\
\text { Elack }\end{array}$ & sfter School & Medium & Medium & Yes & \begin{tabular}{|c|}
$\begin{array}{c}\text { All Other } \\
\text { Types of } \\
\text { Design }\end{array}$ \\
\end{tabular} & Atttudes & No & Other & Missing \\
\hline University of Alabarna & AL & South & Missing & $\begin{array}{c}\text { Boys and } \\
\text { Girls }\end{array}$ & $\begin{array}{c}\text { White and } \\
\text { Elack }\end{array}$ & After School & Low & Low & Yes & $\begin{array}{c}\text { Random } \\
\begin{array}{c}\text { Experimental } \\
\text { Design }\end{array}\end{array}$ & $\begin{array}{l}\text { Behavioral } \\
\text { Intentions }\end{array}$ & No & \begin{tabular}{|c|} 
Difficulty \\
Establishing \\
Stable \\
Schoolicommun \\
ity Partnership \\
\end{tabular} & Evaluation Design \\
\hline Urban League of Eroward $\mathrm{Co}_{0}$ & $\mathrm{FL}$ & South & $3-10$ & $\begin{array}{c}\text { Boys and } \\
\text { Girls }\end{array}$ & Elack Only & A.fter School & Missing & Medium & Missing & \begin{tabular}{|c|} 
All Other \\
Types of \\
Design
\end{tabular} & Attitudes & Yes & $\begin{array}{c}\text { No Challenges } \\
\text { Reported }\end{array}$ & Missing \\
\hline Wheeler County Board of Educ & GA & South & $6-12$ & $\begin{array}{c}\text { Boys and } \\
\text { Girls }\end{array}$ & $\begin{array}{c}\text { White and } \\
\text { Elack }\end{array}$ & After School & Medium & Medium & Yes & $\begin{array}{l}\text { All Other } \\
\text { Types of } \\
\text { Design }\end{array}$ & $\begin{array}{c}\text { Actual } \\
\text { Behavior }\end{array}$ & Yes & Other & Missing \\
\hline Catholic Charities of Sarta Rosa & $\mathrm{CA}$ & West & $6-12$ & $\begin{array}{c}\text { Boys and } \\
\text { Girls }\end{array}$ & $\begin{array}{l}\text { Mostly } \\
\text { White }\end{array}$ & Mixed & Missing & Missing & Yes & \begin{tabular}{|c|}
$\begin{array}{c}\text { Random } \\
\text { Experimental } \\
\text { Design }\end{array}$ \\
\end{tabular} & $\begin{array}{l}\text { Behavioral } \\
\text { Intentions }\end{array}$ & No & $\begin{array}{c}\text { High Staff } \\
\text { Turnover Ratei } \\
\text { Inadequate } \\
\text { Number of Staff }\end{array}$ & Missing \\
\hline Colorado State University & co & West & $5-7$ & $\begin{array}{c}\text { Boys and } \\
\text { Girls }\end{array}$ & Mixed & After School & Low & Medium & Yes & $\begin{array}{c}\text { Random } \\
\text { Experimental } \\
\text { Design }\end{array}$ & $\begin{array}{c}\text { Actual } \\
\text { Behavior }\end{array}$ & Yes & \begin{tabular}{|c|} 
High Staff \\
Turnover Rater \\
Inadequate \\
Number of Staff
\end{tabular} & Missing \\
\hline Northwest Family Services & $\mathrm{OR}$ & West & $7-12$ & $\begin{array}{c}\text { Boys and } \\
\text { Girls }\end{array}$ & Mixed & Mixed & Medium & Missing & Yes & $\begin{array}{c}\text { Quasi- } \\
\text { Experimental } \\
\text { Design }\end{array}$ & $\begin{array}{l}\text { Behavioral } \\
\text { Intentions }\end{array}$ & No & \begin{tabular}{|c|} 
Difficulty \\
Establishing \\
Stable \\
Schoolicommun \\
ity Partnership \\
\end{tabular} & Missing \\
\hline Vista Community Clinic & $\mathrm{CA}$ & West & $2-7$ & $\begin{array}{c}\text { Boys and } \\
\text { Girls }\end{array}$ & $\begin{array}{l}\text { Mostly } \\
\text { Latino }\end{array}$ & After School & Missing & Missing & Yes & \begin{tabular}{|c|} 
Quasi- \\
Experimental \\
Design
\end{tabular} & Attitudes & No & $\begin{array}{c}\text { LowNo } \\
\text { Parental } \\
\text { Involvernent }\end{array}$ & Evaluation Design \\
\hline YMCA of San Diego Co & $\mathrm{CA}$ & West & $7-12$ & $\begin{array}{c}\text { Boys and } \\
\text { Girls }\end{array}$ & Mixed & After School & Low & Missing & Yes & Missing & Missing & Missing & $\begin{array}{l}\text { Recruitment/ } \\
\text { Retention of } \\
\text { Youth } \\
\text { Participants }\end{array}$ & $\begin{array}{l}\text { High Staff } \\
\text { Turnover Ratel } \\
\text { Inadequate } \\
\text { Number of Staff }\end{array}$ \\
\hline
\end{tabular}




\begin{tabular}{|c|c|c|c|c|c|c|c|c|c|}
\hline Organization & State & $\begin{array}{l}\text { Promotes } \\
\text { Bonding }\end{array}$ & \begin{tabular}{|c|} 
Promotes \\
Parent \\
Child \\
Communi- \\
cation
\end{tabular} & \begin{tabular}{|c|} 
\\
Fosters \\
Resilience
\end{tabular} & $\begin{array}{l}\text { Promotes } \\
\text { Social } \\
\text { Competency }\end{array}$ & $\begin{array}{l}\text { Promotes } \\
\text { Emotional } \\
\text { Competency }\end{array}$ & $\begin{array}{c}\text { Promotes } \\
\text { Cognitive } \\
\text { Competency }\end{array}$ & $\begin{array}{c}\text { Promotes } \\
\text { Behavioral } \\
\text { Competency }\end{array}$ & $\begin{array}{c}\begin{array}{c}\text { Promotes } \\
\text { Moral }\end{array} \\
\text { Competency }\end{array}$ \\
\hline Be'er Hagolah Institutes & NY & No & No & Yes & Yes & Yes & No & No & Yes: \\
\hline Boston Medical Center & MA & Yes & Yes & Yes & Yes & Yes & Yes & Yes & Yes \\
\hline Builders for the Farnily \& Youth & NY & Yes & Yes & No & Yes & Yes & Yes & Yes & No \\
\hline Catholic Charities of Onondaga $\mathrm{Co}$ & NY & Yes & Yes & Yes & Yes & Yes: & Yes & Yes & No \\
\hline $\begin{array}{l}\text { Crozer-Chester Medical Center } \\
\text { (Successful.Passages) }\end{array}$ & $\mathrm{PA}$ & Yes & Yes & No & Yes & Yes & Yes & Yes & Yes \\
\hline $\begin{array}{l}\text { Crozer-Chester Medical Center } \\
\text { (Leadership 101) }\end{array}$ & $\mathrm{PA}$ & Yes & Yes & No & Yes & Yes & Yes & Yes & No \\
\hline Educ for Children Families \& Youth & NY & No & No & Yes & Yes & Yes & Yes & Yes & No \\
\hline Mercy Hospital of Pittsburgh & $\mathrm{PA}$ & Yes & Yes & No & Yes & No & Yes & Yes & No \\
\hline St. Luke's Roosevelt Hospital & NY & Yes & Yes & Yes & No & No & No & Yes & No \\
\hline To Our Children's Future with Health & $\mathrm{PA}$ & Yes & Yes & Yes & Yes & Yes: & No & Yes & No \\
\hline University of Maryland Baltimore & MD & Yes & Yes & Yes & Yes & Yes & Yes & Yes: & No \\
\hline YMCA of Cumberland, MD & MD & No & Yes & No & No & No & No & Yes & No \\
\hline Bowling Green State Uniy & $\mathrm{OH}$ & Yes. & Yes. & Yes. & Yes & Yes & Yes & Yes & No \\
\hline Catholic Social Sves of Miami Valley & $\mathrm{OH}$ & Yes & Yes & Yes & Yes & Yes & Yes & Yes & No \\
\hline Lake Co Health Dept & IL & Yes & Yes & Yes & Yes & Yes & Yes & Yes & Yes \\
\hline Pine Ridge Indian Reservation & $\mathrm{SD}$ & Yes & Yes & No & Yes & Yes & Yes & Yes & Yes \\
\hline St. Vincent Mercy Medical Center & $\mathrm{OH}$ & Yes & Yes & Yes & No & No & Yes & No & No \\
\hline Tri County Right to Life Educ Fdn & $\mathrm{OH}$ & Yes & No & Yes & No & Yes: & Yes & No & No \\
\hline Wise Women Gathering Place & $\mathrm{M}$ & Yes & Yes & Yes & No & No & No & No & Yes \\
\hline Youth \& Family Services & SD & Yes & Yes & Yes & Yes & Yes & No & No & No \\
\hline Alabama State University & A.L & Yes. & Yes. & Yes. & No & No & No & No & No \\
\hline Baptist Children's Home Ministries & $\mathrm{TX}$ & Yes & Yes & Yes & Yes & Yes & Yes & Yes & Yes \\
\hline BETA Center & $\mathrm{FL}$ & No & Yes & Yes & No & Yes & Yes & No & No \\
\hline Boat People SOS & $V A$ & No & Yes & Yes & No. & Yes & No & Yes & No \\
\hline Boys and Girls Club of Sarasota & $\mathrm{FL}$ & Yes & Yes & Yes & No & No & Yes & No & Yes \\
\hline Clippers & $\mathrm{NC}$ & No & No & No & No & No & Yes & No & No \\
\hline Dallas Independent School District & $\mathrm{TX}$ & Yes & No & No & No & No & No & No & No \\
\hline Dallas Independent School District 2 & $\mathrm{TX}$ & Yes & No & No & No & No & No & No & No \\
\hline
\end{tabular}




\begin{tabular}{|c|c|c|c|c|c|c|c|c|c|}
\hline \multicolumn{10}{|c|}{$\begin{array}{r}\text { APPENDIX D, PART 2: YOUTH DEVELO } \\
\text { Continued }\end{array}$} \\
\hline Organization & State & $\begin{array}{l}\text { Promotes } \\
\text { Bonding }\end{array}$ & \begin{tabular}{|c|} 
Promotes \\
Parent/ \\
Child \\
Communi- \\
cation
\end{tabular} & \begin{tabular}{|c|} 
\\
Fosters \\
Resilience \\
\end{tabular} & $\begin{array}{l}\text { Promotes } \\
\text { Social } \\
\text { Competency }\end{array}$ & \begin{tabular}{l|} 
Promotes \\
Emotional \\
Competency
\end{tabular} & $\begin{array}{c}\text { Promotes } \\
\text { Cognitive } \\
\text { Competency }\end{array}$ & $\begin{array}{l}\text { Promotes } \\
\text { Behavioral } \\
\text { Competency }\end{array}$ & $\begin{array}{l}\text { Promotes } \\
\text { Moral } \\
\text { Competency }\end{array}$ \\
\hline Fifth Ward Enrichment Program & TX & Yes & Yes & Yes & Yes & No & Yes & Yes & No \\
\hline Healthy Mothers, Healthy Babies & NC & Yes & Yes & Yes & Yes & Yes & Yes & Yes & No \\
\hline Jackson State University & MS & No & No & No & No & No & No & No & No \\
\hline JOVEN & $T X$ & Yes & Yes & No & No. & Yes & Yes & No & No \\
\hline Morehouse School of Medicine & $\mathrm{AL}$ & Yes & Yes & Yes & Yes & Yes & Yes & Yes & Yes \\
\hline Public Health Authority of Cabarrus & $\mathrm{NC}$ & Yes & Yes & Yes & Yes & Yes: & Yes & Yes & No \\
\hline Sisterhood Agenda & NC & No & Yes & No & No & Yes & No & No & No \\
\hline Switchboard of Miami & $\mathrm{FL}$ & No & No & Yes & No & No & Yes & No & No \\
\hline University of Alabama & AL & Yes. & Yes. & Yes. & No & Yes & No & Yes & No \\
\hline Urban League of Broward Co & $\mathrm{FL}$ & Yes & Yes & No & No & No & No & No & No \\
\hline Wheeler County Board of Educ & GA & Yes & Yes & No & No & No & Yes & No & Yes \\
\hline Catholic Charities of Santa Rosa & $\mathrm{CA}$ & Yes & No & Yes & Yes & Yes & Yes & Yes & No \\
\hline Colorado State University & $\infty$ & Yes & Yes & No & No & No & Yes & No & No \\
\hline Northwest Family Services & $O R$ & Yes & Yes & Yes & Yes & Yes & Yes & Yes & Yes \\
\hline Vista Community Clinic & $\mathrm{CA}$ & Yes & Yes & Yes & Yes & Yes & Yes & Yes & Yes \\
\hline YMCA of San Diego Co & $\mathrm{CA}$ & Yes & Yes & Yes & Yes & Yes & Yes & Yes & No \\
\hline
\end{tabular}




\begin{tabular}{|c|c|c|c|c|c|c|c|c|c|c|}
\hline \multicolumn{10}{|c|}{$\begin{array}{r}\text { APPENDIX D, PART 2: YOUTH DEVELO } \\
\text { Continued }\end{array}$} & \multirow[b]{2}{*}{ Total } \\
\hline Organization & State & $\begin{array}{c}\text { Fosters } \\
\text { Self- } \\
\text { determina } \\
\text { tion }\end{array}$ & $\begin{array}{c}\text { Fosters } \\
\text { Spirituality }\end{array}$ & $\begin{array}{l}\text { Fosteres } \\
\text { Self- } \\
\text { Efficacy }\end{array}$ & $\begin{array}{l}\text { Fosters } \\
\text { Clear and } \\
\text { Positive } \\
\text { Identity } \\
\end{array}$ & $\begin{array}{l}\text { Fosters a } \\
\text { Belief in } \\
\text { the Future }\end{array}$ & $\begin{array}{c}\text { Provides } \\
\text { Recogniti } \\
\text { on for } \\
\text { Positive } \\
\text { Behavior } \\
\end{array}$ & $\begin{array}{l}\text { Provides } \\
\text { Opportunities } \\
\text { for Prosocial } \\
\text { Imohement }\end{array}$ & $\begin{array}{c}\text { Fosters } \\
\text { Prosocial } \\
\text { Norms }\end{array}$ & \\
\hline Be'er Hagolah Institutes & NY & Yes & \begin{tabular}{|c|} 
No \\
\end{tabular} & Yes & Yes & Yes & No & No & No & 8 \\
\hline Boston Medical Center & $M A$ & Yes & No & Yes & Yes & Yes & Yes & Yes & Yes & 15 \\
\hline Builders for the Farnily \& Youth & NY & No & No & No & No & No & Yes & Yes & Yes & 9 \\
\hline Catholic Charities of Onondaga Co & NY & Yes & No & Yes & Yes & Yes & No & Yes & Yes & 13 \\
\hline $\begin{array}{l}\text { Crozer-Chester Medical Center } \\
\text { (Successful Passages) }\end{array}$ & $\mathrm{PA}$ & Yes & No & Yes & Yes & Yes & No & Yes & Yes & 13 \\
\hline $\begin{array}{l}\text { Crozer-Chester Medical Center } \\
\text { (Leadership 101) }\end{array}$ & $\mathrm{PA}$ & Yes & No: & Yes: & No & Yes & No & Yes & Yes & 11 \\
\hline Educ for Children Families \& Youth & NY & Yes & No & Yes & No & Yes & No & No & No & 8 \\
\hline Mercy Hospital of Pittsburgh & $\mathrm{PA}$ & No & No & Yes & No & No & No & Yes & Yes & 8 \\
\hline St. Luke's Roosevelt Hospital & NY & No & No & No & No & No & No & No & No & 4 \\
\hline To Our Children's Future with Health & $\mathrm{PA}$ & No & No & Yes: & Yes & No & No & No & No & 8 \\
\hline University of Maryland Baltimore & $\mathrm{MD}$ & Yes & No & Yes & Yes & Yes & No & Yes & Yes & 13 \\
\hline YMCA of Cumberland, MD & MD & No & No & No & No & No & No & No & No & 2 \\
\hline Bowling Green State Uniy & $\mathrm{OH}$ & Yes. & No & Yes & Yes & Yes & No & Yes & Yes & 13 \\
\hline Catholic Social Svcs of Miami Valley & $\mathrm{OH}$ & Yes & No & Yes & Yes & Yes: & No & Yes & Yes & 13 \\
\hline Lake Co Health Dept & $\mathrm{IL}$ & Yes & No & Yes & Yes & Yes & No & Yes & Yes & 14 \\
\hline St. Vincent Mercy Medical Center & $\mathrm{OH}$ & Yes & No & No & Yes & No & No & No: & No & 6 \\
\hline Pine Ridge Indian Reservation & $\mathrm{SD}$ & Yes & Yes & Yes & Yes & No & No & Yes & Yes & 13 \\
\hline Tri County Right to Life Educ Fdn & $\mathrm{OH}$ & No & No & No & Yes & No & No & No & No & 5 \\
\hline Wise Women Gathering Place & $\mathrm{M}$ & No & No & No & No & No & No & Yes & No & 5 \\
\hline Youth \& Family Services & SD & Yes & No & Yes & Yes & Yes & No & Yes & Yes & 11 \\
\hline Alabama State University & AL & No & No & No & No & No & No & No & No & 3 \\
\hline Baptist Children's Home Ministries & $\mathrm{TX}$ & No & No & Yes: & No & Yes & Yes & Yes & No & 12 \\
\hline BETA Center & $\mathrm{FL}$ & No & No & No & Yes & No & No & No & No & 5 \\
\hline Boat People SOS & VA & No & No & Yes & Yes & Yes & Wo & No: & No & 7 \\
\hline Boys and Girls Club of Sarasota & $\mathrm{FL}$ & Yes & No & No & Yes & No & No & Yes & Yes & 9 \\
\hline Clippers & NC & No & No & No & No & No & No & No & No & 1 \\
\hline Dallas Independent School District & $\mathrm{TX}$ & No & No & No & Yes & No & No & No & No & 2 \\
\hline Dallas Independent School District 2 & $\mathrm{TX}$ & No & No & No & Yes & No & No & No & No & 2 \\
\hline
\end{tabular}




\begin{tabular}{|c|c|c|c|c|c|c|c|c|c|c|}
\hline \multicolumn{11}{|c|}{$\begin{array}{r}\text { APPENDIX D, PART 2: YOUTH DEVELO } \\
\text { Continued }\end{array}$} \\
\hline Organization & State & $\begin{array}{c}\text { Fosters } \\
\text { Self- } \\
\text { determina- } \\
\text { tion }\end{array}$ & $\begin{array}{c}\text { Fosters } \\
\text { Spirituality }\end{array}$ & $\begin{array}{l}\text { Fosteres } \\
\text { Self- } \\
\text { Efficacy }\end{array}$ & $\begin{array}{l}\text { Fosters } \\
\text { Clear and } \\
\text { Positive } \\
\text { ldentity }\end{array}$ & $\begin{array}{l}\text { Fosters a } \\
\text { Belief in } \\
\text { the Future }\end{array}$ & $\begin{array}{c}\text { Provides } \\
\text { Recogniti } \\
\text { on for } \\
\text { Positive } \\
\text { Behavior } \\
\end{array}$ & $\begin{array}{l}\text { Provides } \\
\text { Opportunities } \\
\text { for Prosocial } \\
\text { Imvohement }\end{array}$ & $\begin{array}{l}\text { Fosters } \\
\text { Prosocial } \\
\text { Norms }\end{array}$ & Total \\
\hline Fifth Ward Enrichment Program & $\mathrm{TX}$ & Yes & No & Yes & No & Yes & No & Yes & Yes & 11 \\
\hline Healthy Mothers, Healthy Babies & NC & Yes. & No & Yes & Yes & Yes & Yes & Yes & Yes & 14 \\
\hline Jackson State University & MS & No & No & No & No & No & No & No: & No & 0 \\
\hline JOVEN & $T X$ & No & No & Yes & No & Yes & No & No & No & 6 \\
\hline Morehouse School of Medicine & $\mathrm{AL}$ & Yes & No & Yes & Yes & Yes & Yes & Yes & Yes & 15 \\
\hline Public Health Authority of Cabarrus & NC & Yes & No & Yes & Yes & Yes & Yes & Yes & Yes & 14 \\
\hline Sisterhood Agenda & NC & No & No & Yes & No & Yes & Yes & No & No & 5 \\
\hline Switchboard of Miami & $\mathrm{FL}$ & Yes & No & Yes & Yes & No & No & No & No & 5 \\
\hline The Children's Council & $\mathrm{SC}$ & No & No & Yes & Yes & No & No & Yes & No & 8 \\
\hline University of Alabama & AL & Yes. & No & Yes & No & Yes & No & No & Yes & 9 \\
\hline Urban League of Broward $\mathrm{Co}_{0}$ & $\mathrm{FL}$ & Yes & No & No & Yes & Yes & No & No: & No & 5 \\
\hline Wheeler County Board of Educ & GA & No & No & No & No & No & No & Yes & Yes & 6 \\
\hline Catholic Charities of Santa Rosa & $\mathrm{CA}$ & Yes & No & Yes & Yes & Yes & Yes & Yes & Yes & 13 \\
\hline Colorado State University & $\mathrm{co}$ & Yes & No & Yes & No & No & No & No & No & 5 \\
\hline Northwest Family Services & OR & Yes & Yes & Yes & Yes & Yes & No & Yes & Yes & 15 \\
\hline Vista Community Clinic & $\mathrm{CA}$ & Yes & No & Yes & Yes & Yes & No & Yes & Yes & 14 \\
\hline YMCA of San Diego Co & $\mathrm{CA}$ & Yes & No & Yes & Yes & Yes & No & Yes & Yes & 13 \\
\hline
\end{tabular}




\title{
APPENDIX E: SUGGESTED ANNUAL REPORT OUTLINE
}

\author{
Organization Name \\ Program Name \\ Address
}

\section{EXECUTIVE SUMMARY}

Describe the mission of the organization and include an executive summary of the report.

\section{DEMOGRAPHICS OF TARGET AREA AND POPULATION}

- Target area-Describe the socioeconomic characteristics (include statistics) of the catchment area and indicate why this particular area is being targeted for the services.

- Target population-Describe the demographic characteristics (e.g., race, grade level, gender) of the population being targeted

- Include a table they should fill out, so this information is given systematically.

\section{Program characteristics: Project [name]}

- Mission of the program

- Project staff: Explain which project staff members are committed to the program (including position title and responsibilities on the project), percentage of time committed to the project, and the level of training they receive, if applicable. (A table to be filled out would also be helpful here, to promote uniformity of reporting and hence of OAPP review).

- Abstinence education interventions: For each intervention that falls under abstinence education, discuss the following: 
- Name of curriculum or strategy

- Describe any ways in which you have modified, augmented, or altered any of the common curricula you use, including changed content, changed modes of presentation, changed settings of presentation, and incorporated YD objectives or strategies

- Setting (i.e., in school, after school, summer)

○ Target population (i.e., gender, race/ethnicity, other special traits)

- Intensity of intervention, both

- in theory, if a youth participated in everything, and

- in reality, average length of attendance, average frequency of attendance

- Duration—over what period of time—-semester, school year, multiple years

- Frequency-how many sessions typical youth attends per week.

○ Presence of positive youth development goals. if YES, specify which activity attempts to achieve the goal and how:

- Promotes school and community bonding

- Promotes parent-child communication

- Fosters resilience

- Promotes social competencies

- Promotes emotional competencies

- Promotes cognitive competencies

- Promotes behavioral competencies

- Promotes moral competencies

- Fosters self-determination

- Fosters spirituality

- Fosters self-efficacy

- Fosters clear and positive identity

- Fosters belief in the future

- Provides recognition for positive behavior

- Provides opportunities for prosocial involvement

- Fosters prosocial norms

- Youth development interventions: For each intervention that falls under youth development, discuss the following:

- Name of curriculum or strategy

- Setting (i.e., in school, after school, summer)

- Target population (i.e., gender, race/ethnicity, any special traits)

○ Intensity of intervention (i.e., duration, frequency)

- in theory, if a youth participated in everything

- in reality, average length of attendance, average frequency of attendance

○ Presence of positive youth development goals (Catalano et al. 2002; 2004 —if YES, specify which activity attempts to achieve the goal and how: 
- Promotes bonding

- Parent-child communication

- Fosters resilience

- Promotes social competencies

- Promotes emotional competencies

- Promotes cognitive competencies

- Promotes behavioral competencies

- Promotes moral competencies

- Fosters self-determination

- Fosters spirituality

- Fosters self-efficacy

- Fosters clear and positive identity

- Fosters belief in the future

- Provides recognition for positive behavior

- Provides opportunities for prosocial involvement

- Fosters prosocial norms

- Logic model-Include a model that describes how each set of abstinence education and youth development interventions attempt to achieve the program's ultimate goal(s) by detailing the following elements (see exhibits in chapter 2 as examples):

- Intervention components

- Target population

- Moderating factors

- Short-term objectives

○ Long-term objectives

- Goals and progress:

- Short-term goals-Describe each short-term goal, specifying which intervention or activity is going to achieve the goal. Also include specific results, if available.

○ Long-term goals-Describe each long-term goal, specifying which intervention or activity is going to achieve the goal. Also include specific results, if available.

\section{Description of program statistics}

As part of an evaluability assessment, researchers need to know a program's intensity. In addition to knowing the dosage (i.e., duration and frequency) of each intervention, it is also necessary to know and understand the level of participation of each client, including parents or family. Aggregate data on the following may be helpful in determining program intensity: (a) total number of participants who complete 100 percent, 75 percent, and 50 percent of each intervention; (b) total number of parents or families who attend all sessions, 75 percent, and 50 
percent of the sessions; (c) total number of project staff trained; and (d) demographic breakdown (e.g., race, age, gender) of participants and families.

Significant changes from the submitted application

As it is already being done, a description of the changes (e.g., logistical or substantive) that have occurred since the submission of the application is important, as it informs the audience of any challenges or barriers that the program encountered. This section will transition into the next section on challenges.

\section{Challenges and proposed solutions}

Describe all challenges encountered during the year(s) of operation. Also include any proposed solutions.

Evaluation methods

- Evaluator:

- Evaluation research questions: List all questions that the evaluation is trying to address, and indicate via which methods these questions will be addressed.

- Evaluation design/model:

○ Type of evaluation: Describe the quantitative evaluation design you are using, including information on comparison or control groups (present or not, how constructed), pre- and posttesting (what tested, timing of testing), or follow-up testing (what tested, timing of testing).

- Information on qualitative evaluations should also be discussed. For the purpose of an effective and efficient evaluability assessment, it is also important to include information on the different measurable outcomes (e.g., knowledge, attitude, behavior intention, and behavior.

- MIS: Discuss what type of management information system is available and the entire data process, including how the survey is administered, who collects the data and enters them into the system, and who analyzes the data. 
- Evaluation instruments: Include copies of all surveys, questionnaires, protocols, and so on. Describe how administered (group or individual setting, "test" atmosphere or one-onone, answers private or obviously reviewed by tester, etc.).

- Findings for program year: Describe all results. 